\section{OPEN ACCESS}

Edited by:

Salvatore Salomone,

Università degli Studi di Catania, Italy

Reviewed by:

Gustavo Molina,

Universidade Federal dos Vales do Jequitinhonha e Mucuri (UFVJM),

Brazil

Marian Brestic

Slovak University of Agriculture,

Slovakia

${ }^{*}$ Correspondence:

Chandra N. Siddaiah moonnayak@gmail.com

Srinivas Chowdappa

srinivasbub@gmail.com

tThese authors have contributed equally to this work

Specialty section:

This article was submitted to Experimental Pharmacology and Drug

Discovery,

a section of the journal

Frontiers in Pharmacology

Received: 07 November 2017 Accepted: 16 March 2018

Published: 26 April 2018

Citation:

Uzma F, Mohan CD, Hashem A, Konappa NM, Rangappa S, Kamath PV, Singh BP, Mudili V,

Gupta VK, Siddaiah CN,

Chowdappa S, Alqarawi AA and Abd_Allah EF (2018) Endophytic

Fungi-Alternative Sources of

Cytotoxic Compounds: A Review.

Front. Pharmacol. 9:309.

doi: 10.3389/fphar.2018.00309

\title{
Endophytic Fungi-Alternative Sources of Cytotoxic Compounds: A Review
}

\begin{abstract}
Fazilath Uzma ${ }^{1 \dagger}$, Chakrabhavi D. Mohan ${ }^{2 \dagger}$, Abeer Hashem ${ }^{3}$, Narasimha M. Konappa ${ }^{4}$, Shobith Rangappa ${ }^{5}$, Praveen V. Kamath ${ }^{1}$, Bhim P. Singh ${ }^{6}$, Venkataramana Mudili ${ }^{7}$, Vijai K. Gupta ${ }^{8}$, Chandra N. Siddaiah ${ }^{4 *}$, Srinivas Chowdappa ${ }^{1 *}$, Abdulaziz A. Alqarawi ${ }^{9}$ and Elsayed F. Abd_Allah ${ }^{9}$
\end{abstract}

\begin{abstract}
${ }^{1}$ Microbial Metabolite Research Laboratory, Department of Microbiology and Biotechnology, Bangalore University, Bangalore, India, ${ }^{2}$ Department of Studies in Molecular Biology, University of Mysore, Mysore, India, ${ }^{3}$ Botany and Microbiology Department, College of Science, King Saud University, Riyadh, Saudi Arabia, ${ }^{4}$ Department of Studies in Biotechnology, University of Mysore, Mysore, India, ${ }^{5}$ Adichunchanagiri Institute for Molecular Medicine, BG Nagara, Mandya, India, ${ }^{6}$ Molecular Microbiology and Systematics Laboratory, Department of Biotechnology, Mizoram University, Aizawl, India, ${ }^{7}$ Microbiology Division, DRDO-BU-Centre for Life sciences, Bharathiar University, Coimbatore, India, ${ }^{8}$ Department of Chemistry and Biotechnology, ERA Chair of Green Chemistry, School of Science, Tallinn University of Technology, Tallinn, Estonia, ${ }^{9}$ Plant Production Department, College of Food and Agricultural Sciences, King Saud University, Riyadh, Saudi Arabia
\end{abstract}

Cancer is a major cause of death worldwide, with an increasing number of cases being reported annually. The elevated rate of mortality necessitates a global challenge to explore newer sources of anticancer drugs. Recent advancements in cancer treatment involve the discovery and development of new and improved chemotherapeutics derived from natural or synthetic sources. Natural sources offer the potential of finding new structural classes with unique bioactivities for cancer therapy. Endophytic fungi represent a rich source of bioactive metabolites that can be manipulated to produce desirable novel analogs for chemotherapy. This review offers a current and integrative account of clinically used anticancer drugs such as taxol, podophyllotoxin, camptothecin, and vinca alkaloids in terms of their mechanism of action, isolation from endophytic fungi and their characterization, yield obtained, and fungal strain improvement strategies. It also covers recent literature on endophytic fungal metabolites from terrestrial, mangrove, and marine sources as potential anticancer agents and emphasizes the findings for cytotoxic bioactive compounds tested against specific cancer cell lines.

Keywords: bioactive metabolites, anticancer agents, natural compounds, fungal alkaloids, taxol, podophyllotoxin, camptothecin, vinca alkaloids

\section{INTRODUCTION}

Human health is at constant risk due to the occurrence of different types of cancers. Cancer cells are characterized by the enormous replicative potential, apoptotic resistance, and invasive ability. The dislodging of cancer cells from the primary site may lead to the formation of cancer at the secondary site by a process of metastasis which eventually results in the death of an individual. Cancer is a major cause of death globally. An estimated 1,688,780 new cancer cases and 600,920 cancer-related deaths projected to occur in the US in 2017 (Siegel et al., 2017). In 2013, 14.9 million cancer incidences, 8.2 million cancer-related deaths were reported 
(Global Burden of Disease Cancer, 2015). The frequency of cancer-related mortality is increasing in developing countries at an alarming rate. Therefore, several research groups have been actively involved in the development of novel anticancer drugs throughout the globe. It is estimated that the total cost for the development of a new drug is $\sim \$ 2.6$ billion. The global annual expenditure on anticancer drugs is $\sim \$ 100$ billion and is estimated to increase to $\$ 150$ billion by 2020 (Prasad et al., 2017). Many available anticancer drugs exhibit toxicity to proliferating normal cells, possess adverse effects, and are less effective against several types of cancer, which results in a need for bioactive compounds from natural products (Remesh, 2017).

Scientists have begun to comprehend that plants may be reservoirs for an infinite number of microorganisms, commonly referred as endophytes (Schulz et al., 2002; Strobel et al., 2004). Endophytes inhabit at internal plant tissue in a symbiotic association and can spend most of their life cycle within host plants. Approximately one million endophytic species are present in the plant kingdom (Fouda et al., 2015). It is noteworthy to mention that, a small number of endophytic fungi were reported to produce plant growth stimulating hormones such as gibberellic acid and indole acetic acid (Rai et al., 2014). Metabolites produced by endophytes can be influenced by the chemistry of their host plants (Kusari et al., 2012). During the period of co-evolution, some of the endophytes have developed the ability to produce biologically active compounds that are similar or identical to their host plants (Zhao et al., 2011; Jia et al., 2016). Several studies have indicated the possible likelihood of medicinal plants that host endophytic fungi capable of producing pharmacologically important natural products. It is reasonable to postulate that the medicinal properties of these plants could be due to the endophytes residing within them. This has led to an investigation of beneficial compounds related to plant-endophyte interactions (Hardoim et al., 2008). The interaction between plants and endophytes is regulated by genes of both the organisms and controlled by the environmental factors (Moricca and Ragazzi, 2008). In other words, the endophyte may encounter metabolically unfavorable condition due to host defense chemicals (Schulz et al., 1999; Easton et al., 2009). The plant and endophyte association may also modulate the production of secondary metabolites in the host plant. The secondary metabolites isolated from endophytes displayed a broad spectrum of pharmacological properties including anticancer, antiviral, antibacterial, and antifungal activity (Jalgaonwala et al., 2017). In the subsequent sections, we discuss about the anticancer compounds extracted from endophytic fungus from diverse habitats.

\section{NATURAL PRODUCTS AS SOURCES OF NOVEL ANTICANCER COMPOUNDS}

Natural products obtained from endophytic fungi have been identified as continuing and prolific sources of anticancer agents that could have a profound impact on modern medicine for the advancement of anticancer drugs (Schulz et al., 2002; Newman and Cragg, 2007). In the modern era, the discovery of potent medicines with minimal side effects acts as an alternative to conventionally used methods of disease control and treatment (Kusari et al., 2009b; Kusari and Spiteller, 2012). In 2000, it was estimated that $\sim 57 \%$ of compounds in clinical trials for cancer therapy are natural products and their derivatives (Demain and Vaishnav, 2011). Early reports suggest that natural bioactive compounds isolated from endophytes may serve as alternate sources for the discovery of new anticancer drugs (Joseph and Priya, 2011; Alvin et al., 2014; Xie and Zhou, 2017).

Most of the reports dealing with the cytotoxicity of metabolites from endophytic fungi utilize antiproliferative assays in the cancer cell lines and high-throughput screening that essentially allows the evaluation of several compounds together for their potential anticancer activity (Aly et al., 2008; Lei et al., 2013). Isolation of natural bioactive compounds and screening them for the pharmacological properties offer a route for the discovery of drug candidates (Salvador-Reyes and Luesch, 2015). Several endophytic fungal strains have been identified and reported to produce new compounds that are effective in anticancer assays (Stierle and Stierle, 2015). Taxol, vincristine, etoposide, irinotecan, topotecan, and vinblastine are some of that plantderived anticancer drugs that are in clinical use for the treatment of several human cancers (Balunas and Kinghorn, 2005). The review describes the isolation of some of these compounds from endophytes and reports their cytotoxic effect in various cell lines.

\section{CLINICALLY USED ANTICANCER DRUGS AND THEIR PRECURSORS FROM ENDOPHYTIC FUNGI}

Although several natural compounds have been shown to possess good pharmacological and anticancer activities, they often face many challenges. The major challenges in developing natural compounds as drug candidates are identification of the right source; difficulty in extraction due to the excessive degradation by metabolic enzymes; low abundance of active principles; hindrance in large-scale production because of steric complexity high chiral centers, hydrogen bond donors/acceptors, bulky compounds, and diverse aromaticity (Singh et al., 2016). Identification of the good source of natural compounds can be achieved by random selection, folklore, codified systems of medicine, ethnopharmacology, ayurvedic classical texts, or zoopharmacognosy (Singh et al., 2016). Often, plant-derived bioactive natural compounds are of low abundance. For an instance, the total taxol content of the bark of Taxus baccata tree ranges between 0.064 to $8.032 \mathrm{~g} /$ tree, and it was also reported that a tree aged about 100 years can produce the dry bark yield of $5.74 \mathrm{~kg}$ (Nadeem et al., 2002). Therefore, it is essential to develop alternative strategies for the production of bioactive compounds using tissue culture, synthetic/semisynthetic approaches, biotransformation, and use of microbes that can produce desired products in large scale. This review presents bioactive compounds isolated from plant-associated fungal strains obtained from terrestrial, mangrove, and marine habitats, which are capable of inducing cytotoxicity/apoptosis in cancer cells and thereby possess efficient anticancer activity. 


\section{Taxol}

Taxol (1) is the world's first billion-dollar anticancer drug and it is a highly functionalized polycyclic diterpenoid that belongs to a class of taxanes. In 1962, researchers from National Cancer Institute supported project, collected inner bark (phloemcambial tissue) of the Pacific yew tree (Taxus brevifolia) and analyzed for the presence of natural bioactive compounds. Initial screening of crude extract on cancer cells revealed good cytotoxic activity. It took several years to identify and isolate paclitaxel (trade name is taxol) in its pure form from the extract. Thereafter, paclitaxel was identified as a potent antitumor agent and made its way into clinical trials. One of the biggest hurdles faced during the initial days of taxol production is the requirement of six yew trees of 100 years old to treat one cancer patient (Demain and Vaishnav, 2011). In other words, 0.01 to $0.03 \%$ is the taxol content in dry weight of phloem of the yew tree. The constraints in the availability, isolation, and synthesis of taxol made the researchers to think of alternate sources for its production. The efforts resulted in the isolation of 10-deacetylbaccatin III (2) (a precursor for the synthesis of taxol) from T. baccata (European yew). The tree is abundant and bears high amount of 10-deacetyl-baccatin III in its needles and nowadays it is used as a precursor for the synthesis of taxol by semi-synthetic approach (Tulp and Bohlin, 2002). Eventually, FDA approved taxol for the treatment of several types of tumors including breast, ovary, and Kaposi's sarcoma. It is also claimed that taxol is the best-selling cancer drug ever manufactured (Gordon, 2011) with a market size of $\$ 1.6$ billion in 2005 and its structural analog, docetaxel presented the sales of $\$ 3$ billion in 2009 (Demain and Vaishnav, 2011). The efficacy and increased demand for taxol resulted in developing biotechnological approaches to prepare the drug (Kusari et al., 2014). In the present day, taxol is produced by semisynthetic approaches using 10-deacetyl-baccatin III, plant cell culture, and endophytic fungi. In a breakthrough, the T. brevifolia associated endophytic fungus Taxomyces andreanae was reported to produce taxol and related compounds (Stierle et al., 1993). This extraordinary feat led to the discovery of several new taxol-producing endophytic fungi from different host plants (Strobel et al., 1996; Strobel, 2003; Zaiyou et al., 2015). The production of paclitaxel was also identified in an angiosperm named Corylus avellena $\mathrm{L}$ which belongs to the family Betulaceae (Qaderi et al., 2012). In the next section, we have comprehensively discussed the mode of action of taxol in cancer cells, its endophytic fungal sources and cytotoxic ability.
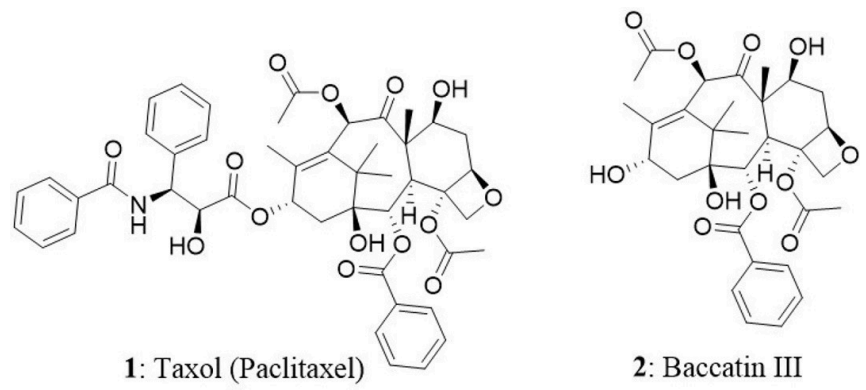

\section{Mode of Action}

Paclitaxel represents a new class of antineoplastic agents and has a unique mode of action. It promotes and stabilizes the polymerization of microtubules and resists their depolymerization. In the presence of taxol, polymerized microtubule is resistant to depolymerization by cold $\left(4^{\circ} \mathrm{C}\right)$ and calcium chloride (4 mM) (Manfredi et al., 1982). This unusual stability of microtubules interfere with the mitotic spindle assembly, chromosome segregation which leads to mitotic arrest and eventually cell death (Schiff et al., 1979; Horwitz et al., 1986; Weaver, 2014).

\section{Endophytic Fungi as Producers of Taxol}

Several research groups have been focusing on the search for new sources of paclitaxel. Several Taxus species, such as Taxus baccata, Taxus chinensis, Taxus cuspidata, Taxus $x$ media, Taxus floridana, Taxus canadensis, Taxus yunnanensis, Taxus mairei, Taxus sumatrana, and Taxus wallichiana, have been reported to produce taxol, albeit with significant variation in the taxane content within and among the different species (Tabata, 2006). Non-Taxus species, such as Cardiospermum halicacabum, Citrus medica, Cupressus sp., Ginkgo biloba, Hibiscus rosa-sinensis, Taxodium distichum, Podocarpus sp., Torreya grandifolia, Terminalia arjuna, and Wollemia nobilis, are also taxol producers (Flores-Bustamante et al., 2010). As discussed earlier, low taxol yields in the plants prompted researchers to discover alternate ways of taxol production which led to the identification of endophytic fungi as sources of taxol (Heinig and Jennewein, 2009). The discovery of taxol-producing endophytes from both Taxus and non-Taxus plants led to screening of array of plants for the identification of endophytes with taxol-producing abilities. Endophytic fungi belonging to the genera Alternaria, Aspergillus, Botryodiplodia, Botrytis, Cladosporium, Ectostroma, Fusarium, Metarhizium, Monochaetia, Mucor, Ozonium, Papulaspora, Periconia, Pestalotia, Pestalotiopsis, Phyllosticta, Pithomyces, and Taxomyces have been tested and reported for the production of paclitaxel and its analogs (Table $\mathbf{1}$ ).

Paclitaxel production from endophytes is reported often in submerged culture (Wang et al., 2001; Zaiyou et al., 2017). Paclitaxel in the fungal extract can be detected by various analytical (Thin layer chromatography, High-performance liquid chromatography), spectroscopic (Matrix-assisted laser desorption/ionization-Time of flight [MALDI-TOF], Nuclear magnetic resonance [NMR], Fast atom bombardment [FAB], Electron spray ionization [ESI]), and immunological techniques (using monoclonal antibodies specific for paclitaxel) (FloresBustamante et al., 2010). Polymerase chain reaction-based methods can be used for the screening and identification of taxol-producing fungi. Previous studies have revealed that presence of genes encoding for the enzymes taxadiene synthase, 10-deacetylbaccatin III-10-O-acetyltransferase, and baccatin III 13-O-(3-amino-3-phenylpropanoyl) transferase could serve as molecular markers for the identification of fungi with taxol-producing abilities (Zhang et al., 2008; Vasundhara et al., 2016). Most taxol production has been reported using a liquid media such as potato dextrose broth (PDB), modified liquid 
TABLE 1 | List of taxol-producing endophytic fungi and their host plants.

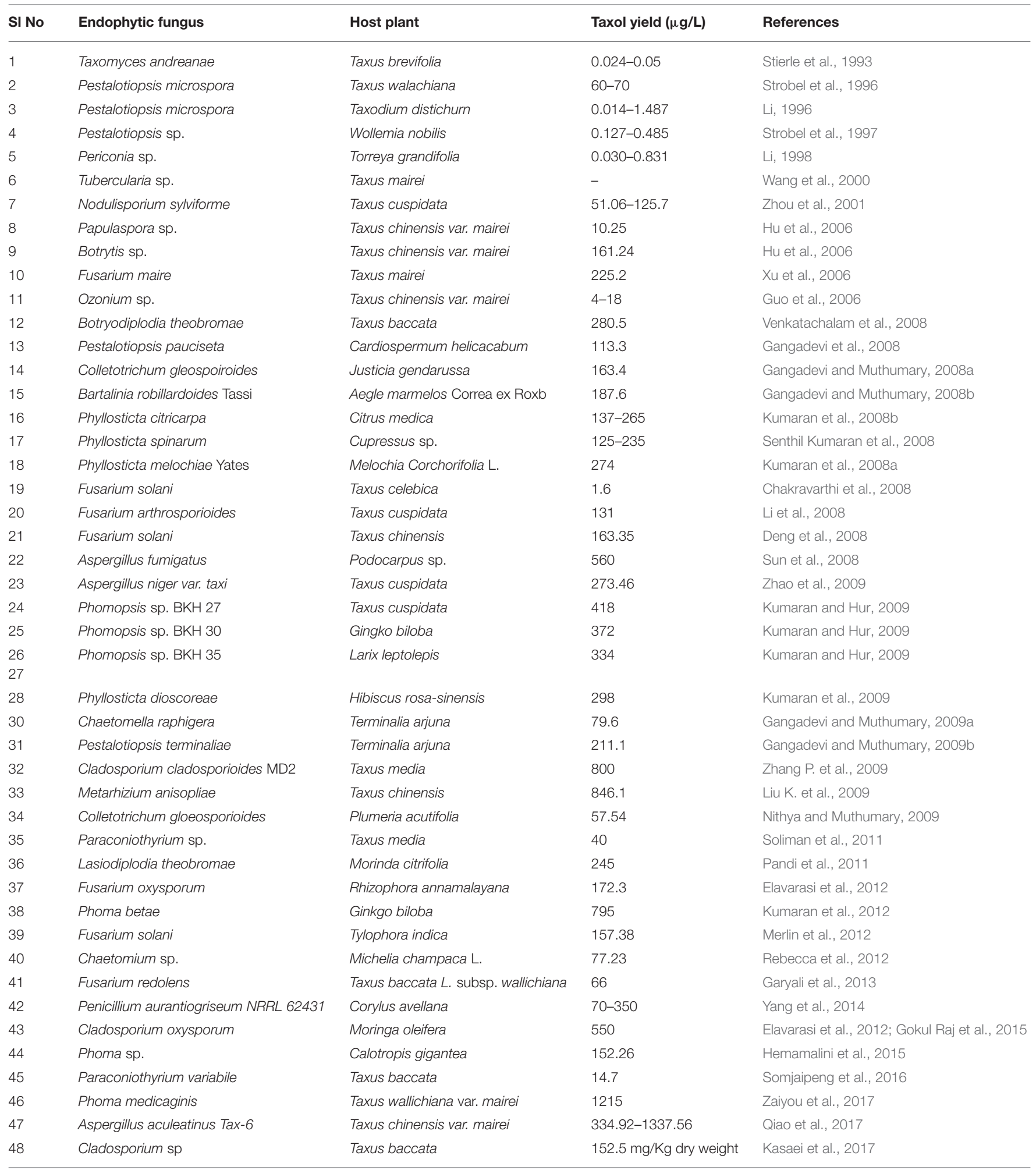

medium (MlD), and S7. However, the highest taxol yield of 846.1 $\mu \mathrm{g} / \mathrm{L}$ was reported from the endophytic fungus, Metarhizium anisopliae of T. chinensis (Liu K. et al., 2009). The endophyte
Cladosporium cladosporioides from Taxus media was also shown to produce $800 \mu \mathrm{g} / \mathrm{L}$ of taxol (Zhang P. et al., 2009). Sun et al. isolated 155 endophytic fungi from the tissue of Podocarpus and 
identified strain A2 as a taxol producer. Subsequently, A2 was classified as Aspergillus fumigates and the taxol yield obtained was $0.56 \mathrm{mg} / \mathrm{L}$ when grown in liquid potato dextrose medium. The isolated taxol inhibited the growth of Vero cells as similar to commercially available taxol (Sun et al., 2008). In another report, 34 endophytic fungi were isolated from the medicinal plant Salacia oblonga at Kigga village, Karnataka, India, and screened for their potential to produce taxol or taxanes. The authors used genomic mining approach to identify the taxolproducing fungus. Among the isolates, seven fungi were found to possess 10 -deacetylbaccatin III-10-O-acetyltransferase gene and one revealed the presence of $\mathrm{C}-13$ phenylpropanoid side chain-CoA acyltransferase gene (Roopa et al., 2015). Recently, 18 fungal isolates were screened for their ability to produce taxol. Cladosporium oxysporum isolated from Moringa oleifera was identified as taxol producing fungus and the taxol yield was found to be $550 \mu \mathrm{g} / \mathrm{L}$. The fungal taxol suppressed the growth of HCT15 with an $\mathrm{IC}_{50}$ value of $3.5 \mu \mathrm{M}$. The presence of gene encoding 10-deacetylbaccatin III-10-O-acetyltransferase confirmed the ability of an endophyte to produce taxol (Gokul Raj et al., 2015). Zaiyou et al. isolated 528 fungal strains from the bark of T. wallichiana var. mairei and only one strain was found to produce paclitaxel. The fungal strain was identified as Phoma medicaginis. The amount of paclitaxel produced when grown in whole potato dextrose broth culture, spent culture medium and dry mycelium was found to be $1.215,0.936 \mathrm{mg} / \mathrm{L}$, and $20 \mathrm{mg} / \mathrm{kg}$, respectively (Zaiyou et al., 2017).

\section{Cytotoxic Ability of Taxol and Its Precursors}

Several cytotoxicity tests have been performed to determine the anticancer abilities of taxol and its precursors. One such interesting investigation highlighted that baccatin III (biosynthetic precursor of taxol) functions via a similar mechanism to taxol. Taxol and baccatin III were extracted from Fusarium solani and tested against HeLa, HepG2, Jurkat-JR4, OVCAR-3, T47D, Jurkat-JR16, and caspase-8-deficient Jurkat cells. Both compounds were able to inhibit cell proliferation of the above-mentioned tumor-derived cell lines with $\mathrm{IC}_{50}$ values between 0.005 to $0.2 \mu \mathrm{M}$ for taxol and 2 to $5 \mu \mathrm{M}$ for baccatin III. These results indicate that although taxol induces apoptosis in the tested cells, there were difference in sensitivity between the tumor cells toward fungal taxol and baccatin III treatment. Among these two compounds, the potent inducer of apoptosis is debatable; because baccatin III is the more active molecule inside the cells during the growth period and is less active than taxol in vitro studies. Conversely, taxol has benefit over baccatin III in cellular uptake, microtubule binding kinetics and interaction with other proteins (Chakravarthi et al., 2013). The endophyte Diaporthe phaseolorum isolated from T. wallichiana var. mairei synthesizes baccatin III. The baccatin III content in PDB and spent culture medium was found to be 0.219 and $0.193 \mathrm{mg} / \mathrm{L}$, respectively (Zaiyou et al., 2013). A detailed description of the taxoid biosynthesis pathway is the solution to improve the supply of taxol and other important taxoids. Several genes and enzymes of the Taxus pathway for the taxoid biosynthesis have now been established (Heinig and Jennewein, 2009). Future studies should focus on the isolation and identification of endophytes that are capable of producing a high amount of taxol, as well as optimization of the production conditions.

\section{Podophyllotoxin}

Podophyllotoxin (3), an aryl tetralin lignan derived from both gymnosperms and angiosperms, has also been investigated as an anticancer drug (Majumder and Jha, 2009); It is widely distributed in the genera Diphylleia, Dysosma, Juniperus, and Sinopodophyllum (also called Podophyllum) (Li et al., 2013). Podophyllotoxin and its analogs are pharmacologically important due to their cytotoxic and antiviral activities (Gordaliza et al., 1994, 2000; Abd-Elsalam and Hashim, 2013). At present, one of the major natural sources of podophyllotoxin is Sinopodophyllum plants. Etoposide (4) and teniposide (5) are the semisynthetic derivatives of podophyllotoxin approved for the treatment of cancer of lungs and testicles, other solid tumors and a variety of leukemias (Kusari et al., 2009a). Etoposide was developed as an alternative to podophyllotoxin in 1966 and received FDA approval in 1983. Subsequently, enzymeassisted asymmetric synthesis of (-)-podophyllotoxin and its C2epimer, (-)-picropodophyllin was described (Berkowitz et al., 2000). However, they are not economically feasible due to the low yield (Chandra, 2012). The supply of podophyllotoxin derivatives from traditional sources is limited due to their low abundance in the plants. A large amount of effort in the past several years has focused on improving production from different podophyllotoxin-producing plant species. Various alternative strategies have been implemented to enhance the production of podophyllotoxin-related compounds. Plant tissue culture is one of the alternate, reliable, and sustainable strategies to produce natural compounds of the plant origin (Ochoa-Villarreal et al., 2016).
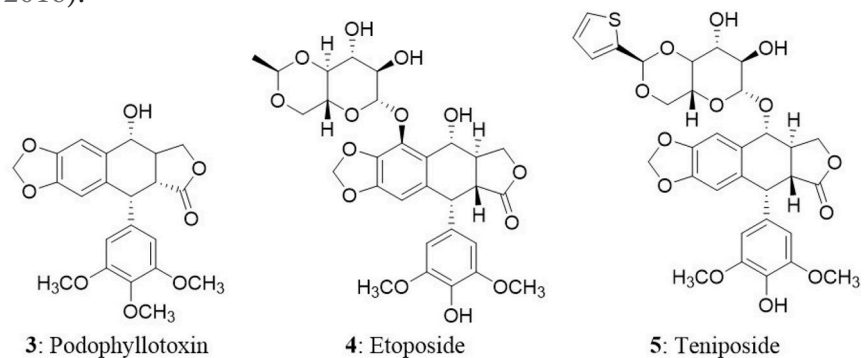

\section{Mode of Action}

Etoposide and teniposide were reported to induce excellent antitumor activity in various cancer models. Their antitumor potential is due to their interaction with the enzyme topoisomerase II (Botta et al., 2001; Cortés and Pastor, 2003). Topoisomerase inhibitors impart their action by two mechanisms-either by eliminating the catalytic activity of topoisomerase II or by increasing the levels of topoisomerase II:DNA covalent complexes (often designated as topoisomerase II poisons) (Nitiss, 2009). Etoposide does not induce cytotoxicity by blocking the catalytic activity of topoisomerase II, but they poison mammalian topoisomerase II by dramatically increasing the covalent DNA cleavage complexes leading to the permanent double-stranded breaks. The increase in enzyme-associated DNA breaks serve as targets for genetic alterations such 
as recombination, exchange of sister chromatid, insertions, deletions, and translocation (Froelich-Ammon and Osheroff, 1995). The accumulation of permanent DNA breaks induces a series of events and drive the cell to death. During this process, topoisomerases serve as a physiological toxins (Van Maanen et al., 1988; Kaufmann, 1989; Hande, 1998; Pendleton et al., 2014).

\section{Podophyllotoxins From Endophytic Fungi}

The two strains of Phialocephala fortinii were isolated from the rhizomes of Podophyllum peltatum which produced podophyllotoxin and the yield ranged between 0.5 and 189 $\mu \mathrm{g} / \mathrm{L}$. The fungal extract was evaluated for cytotoxicity using brine shrimp lethality assay and the $\mathrm{LD}_{50}$ values were in the range of $2-3 \mu \mathrm{g} / \mathrm{mL}$ (Eyberger et al., 2006). Similarly, Fusarium oxysporum, an endophytic fungus isolated from Juniperus recurva in Gulmarg region of South Kashmir, India. The fungus was found to produce podophyllotoxin and the yield was found to be $28 \mu \mathrm{g} / \mathrm{g}$ of dry mass (Kour et al., 2008). In another study, the endophytic fungus Trametes hirsute was isolated from the dried rhizomes of Podophyllum hexandrum which produced podophyllotoxin, podophyllotoxin glycoside and demethoxypodophyllotoxin. The isolated metabolites exhibited cytotoxicity in U-87 cell line (Puri et al., 2006). In another study, Aspergillus fumigatus Fresenius was isolated from Juniperus communis L. Horstmann collected from botanical gardens of Rombergpark, Dortmund, Germany. The fungus was found to produce deoxypodophyllotoxin and maximum yield of $100 \mu \mathrm{g} / \mathrm{g}$ of dry weight of mycelia was observed (Kusari et al., 2009a). In the next study, six fungi were collected from the rhizomes of Sinopodophyllum hexandrum (Royle) Ying plants at Taibai Mountains of China. Among the fungi, Mucor fragilis Fresen. produced podophyllotoxin and kaempferol. The podophyllotoxin yield was found to be $49.3 \mu \mathrm{g} / \mathrm{g}$ of mycelial dry weight (Huang et al., 2014). Recently, first report was published on production of podophyllotoxin from endophyte Alternaria tenuissima. The fungal endophyte was isolated from fresh roots of Sinopodophyllum emodi (Wall.) Ying at Xinglong Mountains, Gansu Province, China. The secondary metabolite analysis revealed the presence of podophyllotoxin in the fungal biomass (Liang et al., 2016). The list of podophyllotoxin producing endophytic fungi and their host plants has been tabulated and presented as Table 2 .

\section{Camptothecin}

Camptothecin (6), an anticancer drug that was first isolated from the bark of the Camptotheca acuminata. It is a pentacyclic pyrroloquinoline alkaloid and a parent compound for clinically used anticancer drugs such as irinotecan and topotecan. It is present in nature in 20-S-camptothecin form and

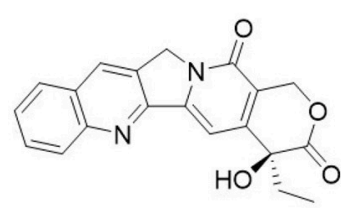

6: Camptothecin

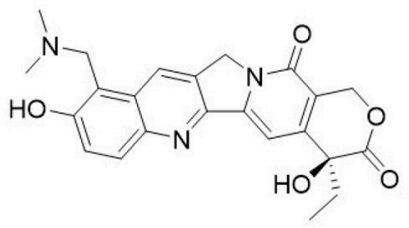

7: Topotecan
20-R-camptothecin enantiomer form is biologically inactive. Camptothecin is produced mainly by $C$. acuminata and Nothapodytes foetida. In National Cancer Institute screening studies, anticancer activity of wood extract of the C. acuminata was identified in 1958. In 1968, Wani and Wall isolated the cytotoxic agent from the extract of C. acuminata and identified the alkaloid as camptothecin (Takimoto, 2002). The purified compound exhibited good cytotoxic activity against several types of tumors. Conversely, clinical development of camptothecin was hampered in clinical trials due to poor water solubility and severe gastrointestinal toxicities (Takimoto, 2002). Eventually, water soluble and biologically active novel camptothecin derivatives were synthesized (Miyasaka et al., 1986). After four decades from the identification of antitumor activity of C. acuminata extract, two camptothecins, topotecan (7) and irinotecan (8) were approved by FDA for the treatment of colorectal cancer, small-cell lung cancer, and ovarian cancer (Blagosklonny, 2004). Meanwhile, camptothecin was identified to target topoisomeraseI in cells to impart its anticancer activity. Several camptothecin derivatives such as IDEC-132 (9-aminocamptothecin), rubitecan (9-nitrocamptothecin), and 10,11-methylenedioxy camptothecin have been identified as good anticancer agents (Ulukan and Swaan, 2002). In the next subsequent sections, we have discussed about the mechanism of action of camptothecin and endophytic fungi as its source.

\section{Mode of Action}

At the initial days, it was believed that camptothecins induce cytotoxicity in cancer cells by inhibiting DNA and RNA synthesis. In the late 1980s, topoisomerase I was identified as the cellular target of camptothecin. Topoisomerase-I is involved in the relaxation of DNA supercoiling that is generated as a result of replication. Topoisomerase-I creates a nick in the single strand of DNA to release supercoiling. In parallel, topoisomerase-I forms an ester linkage with the 3' end of nicked DNA through its catalytic tyrosine to form topoisomerase-I cleavage complexes. Immediately 5'hydroxyl group of the nicked DNA strand nucleophilically attacks the tyrosyl-DNA-phosphodiester bond to bring the DNA to normal topology. In general, topoisomeraseI cleavage complexes are short-lived intermediates and hard to detect in the cellular environment (Pommier, 2006). Camptothecin and its derivatives interact with topoisomeraseI cleavage complexes and stabilize them. This results in the initiation of series of apoptotic events, finally leading to cell death. Studies using mutant yeasts (Saccharomyces cerevisiae and Schizosaccharomyces pombe) that lacks topoisomerase I activity were completely resistant to camptothecin indicating the absence of off-targets (Eng et al., 1988). It is now clearly demonstrated that the antitumor activity of camptothecin is due to its ability to inhibit DNA topoisomerase- I, which is involved

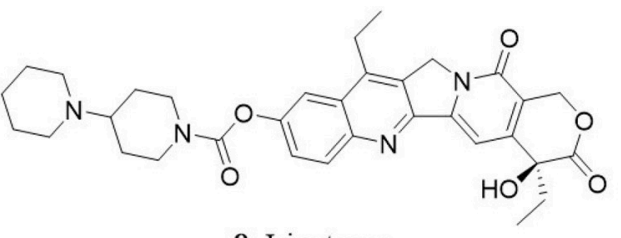

8: Irinotecan 
TABLE 2 | List of podophyllotoxin producing endophytic fungi and their host plants.

\begin{tabular}{|c|c|c|c|c|}
\hline SI No & Endophytic fungus & Host plant & Podophyllotoxin yield ( $\mu \mathrm{g} / \mathrm{L})$ & References \\
\hline 1 & Alternaria sp. & Podophyllum hexandrum & - & Chandra, 2012 \\
\hline 2 & Phialocephala fortinii & Podophyllum peltatum & $0.5-189$ & Eyberger et al., 2006 \\
\hline 3 & Trametes hirsute & Podophyllum hexandrum & $30 \mu \mathrm{g} / \mathrm{g}$ dry weight of mycelia & Puri et al., 2006 \\
\hline 4 & Alternaria neesex Ty & Sinopodophyllum hexandrum & 2.418 & Cao et al., 2007 \\
\hline 5 & Fusarium oxysporum & Juniperus recurva & $28 \mu \mathrm{g} / \mathrm{g}$ dry weight of mycelia & Kour et al., 2008 \\
\hline 6 & Alternaria sp. & Sabina vulgaris & - & Zhao et al., 2010b \\
\hline 7 & Alternaria sp. & Podophyllum hexandrum & - & Zhao et al., 2010b \\
\hline 8 & Monilia sp. & Dysosma veitchii & - & Zhao et al., 2010b \\
\hline 9 & Penicillium sp. & Podophyllum hexandrum & - & Zhao et al., 2010b \\
\hline 10 & Penicillium sp. & Diphylleia sinensis & - & Zhao et al., 2010b \\
\hline 11 & Fusarium solani & Podophyllum hexandrum & $29 \mu \mathrm{g} / \mathrm{g}$ dry weight of mycelia & Nadeem et al., 2012 \\
\hline 12 & Alternaria tenuissima & Sinopodophyllum emodi (Wall.) Ying & $\begin{array}{l}\text { Chloroform extract: } 50.5 \mathrm{mg} \text {; Butanol } \\
\text { extract: } 348 \mathrm{mg} \text {; Methanol extract of the } \\
\text { mycelia: } 1139.9 \mathrm{mg}\end{array}$ & Liang et al., 2016 \\
\hline \multirow[t]{2}{*}{13} & Chaetomium globosum & Sinopodophyllum hexandrum & - & Wang et al., 2017 \\
\hline & Pseudallescheria sp. & & & \\
\hline
\end{tabular}

in the swiveling and relaxation of DNA during replication and transcription.

\section{Camptothecin From Endophytic Fungi}

Camptothecin obtained from plant sources does not meet the requirement from the global market and consistent attempts have been underway to identify novel sources of camptothecin. Entrophospora infrequens is the endophytic fungus isolated from the inner bark of $N$. foetida in Konkan ghats, West coast of India. The yield of camptothecin was found to be $18 \mu \mathrm{g} / \mathrm{mg}$ of the chloroform extract. The isolated fungal camptothecin showed good cytotoxicity against A549, HEp2, and OVCAR-5 (Puri et al., 2005). In another study, the same fungus produced $4.96 \mathrm{mg}$ of camptothecin per $100 \mathrm{~g}$ of dry mass in $48 \mathrm{~h}$ in a bioreactor (Amna et al., 2006). The two endophytic fungal strains of $F$. solani were isolated from Apodytes dimidiate in the Western Ghats, India. In broth culture after 4 days of incubation, the yield of camptothecin produced by two strains was found to be 37 and $53 \mu \mathrm{g} / 100 \mathrm{~g}$ (Shweta et al., 2010). In another study, Shweta et al. isolated three endophytic fungi (A. alternate, Fomitopsis sp., Phomopsis sp.) from fruit and seeds of Miquelia dentata and found to produce camptothecin, 9-methoxycamptothecin, and 10-hydroxycamptothecin. The yield of camptothecin obtained from A. alternata, Fomitopsis sp., and Phomopsis sp. was 73.9, 55.49 , and $42.06 \mu \mathrm{g} / \mathrm{g}$ dry weight (Shweta et al., 2013). Three camptothecin-producing fungi, Aspergillus sp. LY341, Aspergillus sp. LY355, and Trichoderma atroviride LY357 were isolated from C. acuminata. The corresponding camptothecin yields were $7.93,42.92$, and $197.82 \mu \mathrm{g} / \mathrm{L}$, respectively. Unfortunately, LY341 and LY355 strains lost the camptothecin-producing capability with repetitive subculturing. On the other hand, consistent production of camptothecin was seen in LY357 from second to eighth generation ( $\mathrm{Pu}$ et al., 2013; Kai et al., 2015).
In the next study, 161 fungi were isolated from C. acuminata. Among the isolates, Botryosphaeria dothidea X-4 fungus was reported to produce 9-methoxycamptothecin (Ding et al., 2013). The endophytic fungi Neurospora sp. was isolated from the seed of $N$. foetida and found to produce camptothecin. The isolated fungal camptothecin was tested against the A549 and OVCAR-5 cells and it showed good cytotoxicity in both the cell lines (Rehman et al., 2008). In another study, the fungus (XZ-01) that belongs to Phomopsis sp. was isolated from twigs of C. acuminata collected from the Jiangshi Natural Reserve, Fujian, China. Details of the camptothecin-producing fungi and their corresponding host plants are provided in Table 3.

\section{Vinca Alkaloids}

The success story of isolation of vinca alkaloids from Madagascar periwinkle (botanical name is Catharanthus roseus G. Don and commonly called Vinca rosea) marks back to late 1950s. In Jamaican folklore, the extracts of periwinkle were used as an oral hypoglycemic agent in the absence of insulin treatment (Noble, 1990). Eventually, two groups (Noble, Beer, and Cutts at the Collip Laboratories; Svoboda, Johnson, Neuss, and Gonman at the Lilly Research Laboratories) investigated the antidiabetic property of periwinkle extracts but did not find substantial hypoglycemic activity. The intense research on the extracts of periwinkle led to the isolation of an alkaloid, which reduced the WBCs and depleted bone marrow in rats. The new alkaloid was named as vincaleukoblastine because of its origin and effect on WBCs; subsequently named as vinblastine. Thorough phytochemical investigation of the extract over a period of a decade led to the discovery of anticancer alkaloids including vinblastine (9), vincristine (10), vinleunosine, and vinrosidine (Johnson et al., 1963; Noble, 1990, 2016). The vinca alkaloids are terpenoid indoles obtained from the coupling of catharanthine monomer and vindoline and are mainly used in chemotherapy 


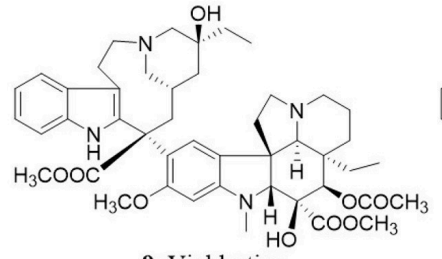

9: Vinblastine

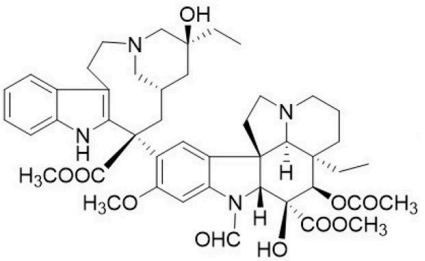

10: Vincristine regimens due to their ability to reduce the number of white blood cells in acute lymphoblastic leukemia and nephroblastoma. Vinca alkaloids represent the second most used class of anticancer drugs in the treatment of various malignancies (Moudi et al., 2013).

\section{Mode of Action}

Vinca alkaloids target cell cycle progression by interfering with activities of the microtubule cytoskeleton. Microtubules are the cytoskeletal elements made up of tubulin heterodimers which are associated with transportation of cargo within the cell, motility of the cell itself, and progression of the cell cycle (Downing, 2000). In the structural organization of microtubules, $\alpha$ and $\beta$ tubulins arrange themselves alternatively to form a protofilament and $\alpha$ subunits are projected toward minus end and $\beta$ subunits toward plus end (confers polarity). Further, protofilaments arrange in a parallel fashion to form a hollow cylindrical structure called microtubule (Kirschner, 1980; Dumontet and Jordan, 2010). The microtubules grow and shrink rapidly by addition and removal of tubulin subunits by a phenomenon referred as dynamic instability of microtubules. The free subunits are added up at plus end with the subsequent release of subunits at minus end which results in the steady-state maintenance of microtubule length (Alberts, 2017). The shortening and lengthening of a microtubule are essential to pull sister chromosomes during mitosis and push out membranes respectively (Wilson et al., 1999). Impeding the dynamics and stability of microtubules can block cell division in multiple ways. Vinca alkaloids are found to interact with the vinca domain of $\beta$ tubulin in order to destabilize the microtubule structure. The binding site is present toward the plus end of microtubule and vinblastine suppresses dynamic instability at plus ends (Wilson et al., 1999). Previous findings suggested that vinca derivatives inhibit cell cycle at metaphase of mitosis (Jordan et al., 1991). They bind to tubulin intracellularly, resulting in the subsequent dissolution of microtubules, and prevent cells from assembling the spindles needed for the division, thus arresting cells in mitosis, which is necessary to mediate their cytotoxic effects (Jordan et al., 1991; Newman and Cragg, 2007). Primarily vinca alkaloids interfere with the formation of microtubule and mitotic spindle dynamics coupled with the disruption of intracellular transport. Overall, vinca alkaloids impart their antineoplastic effect by serving as microtubuledestabilizing agents. Vincristine and vinblastine are approved for the treatment of Hodgkin lymphoma and structural analogs of vinca alkaloids (Vinflunine, Vinorelbine, Anhydrovinblastine) targeting tubulin polymerization are in phase-II/III trials for the treatment of breast cancer and carcinoma (Kaur et al., 2014).

\section{Vinca Alkaloids From Endophytic Fungi}

An endophytic fungus, Alternaria sp., isolated from the phloem of Catharanthus roseus was reported to produce vinblastine (Guo et al., 1998). Later, the endophyte, F. oxysporum isolated from the phloem of $C$. roseus was found to produce vincristine (Zhang et al., 2000). An endophytic fungus from the leaves of $C$. roseus was also reported to produce vincristine (Yang et al., 2004). The endophytic fungus, F. solani from $C$. roseus was screened for vinca alkaloids using thin layer chromatography and electron spray ionization-mass spectroscopic analysis. The fungus was identified to produce vincristine and vinblastine (Kumar et al., 2013). The amount of vinblastine and vincristine in the culture filtrate was found to be 76 and $67 \mu \mathrm{g} / \mathrm{L}$, respectively. The endophytic fungus Talaromyces radicus from $C$. roseus produced $670 \mu \mathrm{g} / \mathrm{L}$ of vincristine in modified M2 medium and $70 \mu \mathrm{g} / \mathrm{L}$ of vinblastine in PDB medium. Vincristine was partially purified and evaluated for cytotoxicity in HeLa, MCF7, A549, U251, and A431 cells. The treatment of vincristine resulted in the dose-dependent growth inhibition in HeLa, MCF7, A549, U251, and $A 431$ with $\mathrm{IC}_{50}$ values of $4.2,4.5,5.5,5.5$, and $5.8 \mu \mathrm{g} / \mathrm{mL}$, respectively. However, the normal cells (HEK293) were not significantly affected (Palem et al., 2015).

\section{CYTOTOXIC COMPOUNDS FROM ENDOPHYTIC FUNGI ASSOCIATED WITH TERRESTRIAL PLANTS}

The secondary metabolites from endophytic fungi have been widely recognized as potent antitumor agents. The secondary metabolite and bioactive compounds from endophytic fungi are screened for their antiproliferative activity against different cancer cell lines (Please refer Tables 4-6 for examples). Preliminary screening of extracts or compounds using cytotoxicity assays allows the rapid identification of compounds with anticancer activity. It is also essential to identify compounds that discriminate between cancer and normal cells, and specifically induce cytotoxicity in cancer cells. Specific cytotoxicity is imperative in cancer therapy, as the standard chemotherapy often affects actively dividing normal cells. However, few anticancer agents may be carcinogenic (Blagosklonny, 2005). Hence, toxicity screening is one of the major steps employed to identify anticancer agents (Parasuraman, 2011). In the next section, compounds isolated from endophytic fungi associated with terrestrial plants and their anticancer potential have been discussed.

Two new xanthone dimers, phomoxanthone A (11) and B (12) were isolated from the endophytic fungus Phomopsis sp., BCC 1323 from Tectona grandis at Mee Rim district, Chiang Mai Province, Northern Thailand. The compounds exhibited remarkable cytotoxicity towards $\mathrm{KB}, \mathrm{BC}-1$ cells, and

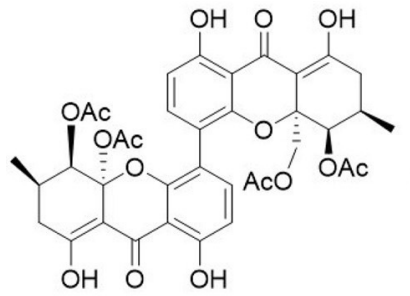

11: Phomoxanthone A

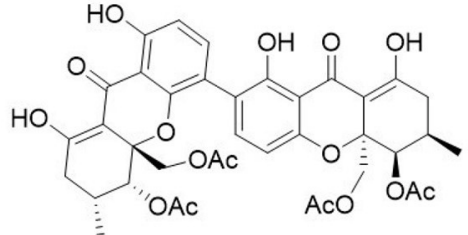

12: Phomoxanthone $B$ 
TABLE 3 | List of camptothecin, 9-methoxycamptothecin, 10-hydroxycamptothecin producing endophytic fungi, and their host plants.

\begin{tabular}{|c|c|c|c|c|}
\hline SI No & Endophytic fungus & Host plant & Camptothecin yield $(\mu \mathrm{g} / \mathrm{g})$ & References \\
\hline 1 & Entrophospora infrequens & Nothapodytes foetida & $18 \mu \mathrm{g} / \mathrm{mg}$ of the chloroform extract & Puri et al., 2005 \\
\hline 2 & Entrophospora infrequens & Nothapodytes foetida & 49.6 & Amna et al., 2006 \\
\hline 3 & Neurospora crassa & Nothapodytes foetida & - & Rehman et al., 2008 \\
\hline 4 & Fusarium solani & Camptotheca acuminata & - & Kusari et al., 2009b \\
\hline 5 & Fusarium solani & Apodytes dimidiate & 0.37 & Shweta et al., 2010 \\
\hline 6 & Fusarium solani & Apodytes dimidiate & 0.53 & \\
\hline 7 & Trichoderma atroviridae & Camptotheca acuminata & $197.82 \mu \mathrm{g} / \mathrm{L}$ & Pu et al., 2013 \\
\hline 8 & Botryosphaeriadothidea & Camptotheca acuminata & - & Ding et al., 2013 \\
\hline \multirow[t]{4}{*}{9} & Fusarium nematophilum & Camptotheca acuminata & 37 & Su et al., 2014 \\
\hline & Alternaria alternata & & 29 & \\
\hline & Phomopsis vaccinii & & 24 & \\
\hline & Colletotrichum gloeosporioides & & 17 & \\
\hline \multirow[t]{2}{*}{10} & Fusarium solani & Camptotheca acuminata & $40 \mu \mathrm{g} / \mathrm{g}$ & Ran et al., 2017 \\
\hline & & & $150 \mu \mathrm{g} / \mathrm{L}$ & \\
\hline
\end{tabular}

non-malignant Vero cells. Phomoxanthone A was relatively more potent than phomoxanthone $\mathrm{B}$ with $\mathrm{IC}_{50}$ values of $0.99,0.51$, and $1.4 \mu \mathrm{g} / \mathrm{mL}$ against $\mathrm{KB}, \mathrm{BC}-1$, and Vero cells, respectively. Moreover, phomoxanthone $\mathrm{A}$ and $\mathrm{B}$ displayed selectively potent cytotoxicity in BC- 1 cells with $\mathrm{IC}_{50}$ values 0.51 and $0.70 \mu \mathrm{g} / \mathrm{mL}$, respectively (Isaka et al., 2001).

A new species of Acremonium was isolated from the twig of Knema laurina (Thai medicinal plant) in the forest areas of Nakhon Ratchasima Province, Thailand. The endophytic fungus was found to produce brefeldin A (13) 8-deoxytrichothecin, trichothecolone, 7-hydroxytrichodermol, and 7-hydroxyscirpene. Brefeldin A exhibited potent cytotoxic activity against $\mathrm{KB}, \mathrm{BC}-1$, and $\mathrm{NCI}-\mathrm{H} 187$ with $\mathrm{IC}_{50}$ values of $0.18,0.04$, and $0.11 \mu \mathrm{M}$, respectively (Chinworrungsee et al., 2008). Altersolanol (14), a hydroxylated tetrahydro anthraquinone, macrosporin (15), and 1,2,4,5-tetrahydroxy7-methoxy-2-methyl-1,2,3,4-tetrahydroanthracene-9,10-dione (16) were isolated from an Alternaria endophytic fungus. The fungus is collected from a healthy leaf of Erythrina variegata in Samutsakorn Province, Thailand. Altersolanol showed potent antiangiogenic activity by inhibiting endothelial cell proliferation, tube formation, and migration. Altersolanol also suppressed the formation of blood vessels in ex vivo and in vivo models. Taken together, altersolanol was found to be a good antiangiogenic agent and a promising candidate in development of therapeutics against cancer and other pro-angiogenesis-related diseases (Pompeng et al., 2013).
The endophytic fungus Preussia sp., was obtained from a mature stem of Aquilaria sinensis (Thymelaeaceae) collected from Guangxi Medicinal Arboretum. The endophyte produced three spirobisnaphthalenes (spiropreussione A, spiropreussione $\mathrm{B}$, and spiropreussomerin A) possessing two naphthalenederived C-10 units' bridges through a spiroketal linkage. Among the novel spirobisnaphthalenes, spiropreussione A (17) exhibited in vitro cytotoxic activity against the A2780 and the BEL-7404 cell lines with $\mathrm{IC}_{50}$ values of 2.4 and $3.0 \mu \mathrm{M}$, respectively. However, spiropreussione A was less active against the HCT-8, BGC-823, and $\mathrm{A} 549$ cell lines with $\mathrm{IC}_{50}$ values more than $10 \mu \mathrm{M}$ (Chen et al., 2009). The endophytic fungus Eutypella sp. from Etlingera littoralis produced two new $\gamma$-lactones (eutypellins A and B), two ent-eudesmane sesquiterpenes (ent-4(15)-eudesmen11-ol-1-one and ent-4(15)-eudesmen-1R,11-diol), and three known pimarane diterpenes (diaporthein $\mathrm{B}$, scopararane $\mathrm{A}$, and libertellenone $\mathrm{C}$ ). Among the isolated compounds, ent-4(15)-eudesmen-11-ol-1one (18) showed weak cytotoxicity against NCI-H187, MCF7, $\mathrm{KB}$, and Vero cell lines with $\mathrm{IC}_{50}$ values of $11,20,32$, and $32 \mu \mathrm{M}$, respectively (Isaka et al., 2009). In another study, seven novel eremophilane-type sesquiterpenes and the known mairetolide F were isolated from the endophyte Xylaria sp. The fungus was isolated from Licula spinose at Trang Province, Thailand. The compounds eremophilanolide 1, 2 and 3 (19-21) exhibited moderate cytotoxicity with $\mathrm{IC}_{50}$ values ranging between 3.8 and $21 \mu \mathrm{M}$ against the KB, MCF7, and NCI-H187 cell lines (Isaka et al., 2010).<smiles>COc1cc(O)c2c(c1)C(=O)c1cc(OC)cc3c1C(=O)C1=C(C3=O)[C@@H](O)[C@@H](O)[C@](C)(O)[C@H](O)[C@H]1C2=O</smiles> 
TABLE 4 | List of cytotoxic compounds isolated from endophytic fungi of terrestrial habitats.

\begin{tabular}{|c|c|c|c|c|c|c|}
\hline $\begin{array}{l}\text { SI } \\
\text { No }\end{array}$ & Host plant & Fungal endophyte & $\begin{array}{l}\text { Isolated cytotoxic } \\
\text { compound/s }\end{array}$ & Tested cell line/s & Cytotoxicity & $\begin{array}{l}\text { References \& } \\
\text { Units of } \\
\text { cytotoxicity }\end{array}$ \\
\hline \multirow[t]{6}{*}{1} & \multirow[t]{6}{*}{ Tectona grandis } & \multirow[t]{6}{*}{ Phomopsis sp. } & \multirow[t]{3}{*}{ Phomoxanthone A } & $\mathrm{KB}$ & 0.99 & \multirow{6}{*}{$\begin{array}{l}\text { Isaka et al., } 2001 \\
\mathrm{IC}_{50}(\mu \mathrm{g} / \mathrm{mL})\end{array}$} \\
\hline & & & & $B C-1$ & 0.51 & \\
\hline & & & & Vero & 1.4 & \\
\hline & & & \multirow[t]{3}{*}{ Phomoxanthone B } & $\mathrm{KB}$ & 4.1 & \\
\hline & & & & BC-1 & 0.7 & \\
\hline & & & & Vero & 1.8 & \\
\hline \multirow[t]{15}{*}{2} & \multirow[t]{15}{*}{ Knema laurina } & \multirow[t]{15}{*}{ Acremonium sp. } & \multirow[t]{3}{*}{ Brefeldin A } & $\mathrm{KB}$ & 0.18 & \multirow{15}{*}{$\begin{array}{l}\text { Chinworrungsee } \\
\text { et al., } 2008 \\
\mathrm{IC}_{50}(\mu \mathrm{M})\end{array}$} \\
\hline & & & & $\mathrm{BC}-1$ & 0.04 & \\
\hline & & & & $\mathrm{NCl}-\mathrm{H} 187$ & 0.11 & \\
\hline & & & \multirow[t]{3}{*}{ 8-Deoxy-trichothecin } & $\mathrm{KB}$ & $>62.81$ & \\
\hline & & & & $\mathrm{BC}-1$ & 0.88 & \\
\hline & & & & $\mathrm{NCl}-\mathrm{H} 187$ & 1.48 & \\
\hline & & & \multirow[t]{3}{*}{ Trichothecolone } & $\mathrm{KB}$ & 12.90 & \\
\hline & & & & BC-1 & 10.06 & \\
\hline & & & & $\mathrm{NCl}-\mathrm{H} 187$ & 11.31 & \\
\hline & & & \multirow[t]{3}{*}{$7 \alpha$-Hydroxytrichodermol } & $\mathrm{KB}$ & $>75.10$ & \\
\hline & & & & $\mathrm{BC}-1$ & 2.37 & \\
\hline & & & & $\mathrm{NCl}-\mathrm{H} 187$ & 1.73 & \\
\hline & & & \multirow[t]{3}{*}{$7 \alpha$-Hydroxyscirpene } & $\mathrm{KB}$ & 8.47 & \\
\hline & & & & $B C-1$ & 21.53 & \\
\hline & & & & $\mathrm{NCl}-\mathrm{H} 187$ & 27.76 & \\
\hline \multirow[t]{5}{*}{3} & \multirow[t]{5}{*}{ Polygonum senegalense } & \multirow[t]{5}{*}{ Alternaria sp. } & Alternariol & L5178Y & 1.7 & \multirow{5}{*}{$\begin{array}{l}\text { Aly et al., } 2008 \\
\text { EC }_{50}(\mu \mathrm{g} / \mathrm{mL})\end{array}$} \\
\hline & & & Alternariol 5-O-sulfate & & 4.5 & \\
\hline & & & Alternariol 5-O-methyl ether & & 7.8 & \\
\hline & & & Altenusin & & 6.8 & \\
\hline & & & Desmethylaltenusin & & 6.2 & \\
\hline 4 & Platycladus orientalis & Phyllosticta spinarum & Tauranin & $\mathrm{NCl}-\mathrm{H} 460$ & 4.3 & Wijeratne et al., \\
\hline & & & & MCF7 & 1.5 & 2008 \\
\hline & & & & SF-268 & 1.8 & $\mathrm{IC}_{50}(\mu \mathrm{M})$ \\
\hline & & & & PC-3M & 3.5 & \\
\hline & & & & MIA Pa Ca-2 & 2.8 & \\
\hline 5 & Glochidion ferdinandi & Eupenicillium & Trichodermamide C & HCT116 & 0.68 & Davis et al., 2008 \\
\hline & & sp. & & A549 & 4.28 & $\mathrm{IC}_{50}(\mu \mathrm{M})$ \\
\hline 6 & Hopea hainanensis & Penicillium sp. & Monomethylsulochrin & $\mathrm{KB}$ & 30.0 & Wang et al., 2008 \\
\hline & & & & HepG2 & 30.0 & $\mathrm{IC}_{50}(\mu \mathrm{g} / \mathrm{mL})$ \\
\hline & & & Rhizoctonic acid & $\mathrm{KB}$ & 20.0 & \\
\hline & & & & HepG2 & 25.0 & \\
\hline & & & Asperfumoid 3 & $\mathrm{~KB}$ & 20.0 & \\
\hline & & & & HepG2 & 15.0 & \\
\hline & & & 3,5-Dichloro-p-anisic acid 6 & $\mathrm{~KB}$ & 5.0 & \\
\hline & & & & HepG2 & 10.0 & \\
\hline 7 & Aquilaria sinensis & Preussia sp. & Spiropreussione A & A2780 & 2.4 & Chen et al., 2009 \\
\hline & & & & BEL-7404 & 3.0 & $\mathrm{IC}_{50}(\mu \mathrm{M})$ \\
\hline 8 & Etlingera littoralis & Eutypella sp. & Ent-4(15)-eudesmen-11-ol- & $\mathrm{NCl}-\mathrm{H} 187$ & 11 & Isaka et al., 2009 \\
\hline & & & 1-one & MCF7 & 20 & $\mathrm{IC}_{50}(\mu \mathrm{M})$ \\
\hline & & & & $\mathrm{KB}$ & 32 & \\
\hline & & & & Vero & 32 & \\
\hline 9 & Mimosops elengi & Ascomycetes & Ergoflavin & $\mathrm{ACHN}$ & 1.2 & Deshmukh et al., \\
\hline & & & & $\mathrm{NCl}-\mathrm{H} 460$ & 4.0 & 2009 \\
\hline & & & & Panc1 & 2.4 & $\mathrm{IC}_{50}(\mu \mathrm{M})$ \\
\hline & & & & НCT116 & 8.0 & \\
\hline & & & & Calu1 & 1.5 & \\
\hline
\end{tabular}


TABLE 4 | Continued

\begin{tabular}{|c|c|c|c|c|c|c|}
\hline $\begin{array}{l}\text { SI } \\
\text { No }\end{array}$ & Host plant & Fungal endophyte & $\begin{array}{l}\text { Isolated cytotoxic } \\
\text { compound/s }\end{array}$ & Tested cell line/s & Cytotoxicity & $\begin{array}{l}\text { References \& } \\
\text { Units of } \\
\text { cytotoxicity }\end{array}$ \\
\hline 11 & Camellia sinensis & Pestalotiopsis fici & Pestaloficiol L & $\begin{array}{l}\text { HeLa } \\
\text { MCF7 }\end{array}$ & $\begin{array}{l}8.7 \\
17.4\end{array}$ & $\begin{array}{l}\text { (Liu L. et al., 2009) } \\
\text { IC } \mathrm{C}_{50}(\mu \mathrm{M})\end{array}$ \\
\hline 12 & Roystonea regia & $\begin{array}{l}\text { Pestalotiopsis } \\
\text { photiniae }\end{array}$ & $\begin{array}{l}\text { Photinides A } \\
\text { Photinides B } \\
\text { Photinides C } \\
\text { Photinides D } \\
\text { Photinides E } \\
\text { Photinides F }\end{array}$ & MDA-MB-231 & $\begin{array}{l}24.4 \\
24.2 \\
23.1 \\
24.4 \\
24.6\end{array}$ & $\begin{array}{l}\text { Ding et al., } 2009 \\
\text { (\% inhibitory rate at } \\
10 \mu \mathrm{g} / \mathrm{mL})\end{array}$ \\
\hline 14 & Ginkgo biloba & Chaetomium globosum & $\begin{array}{l}\text { Chaetomugilin A } \\
\text { Chaetomugilin D } \\
\text { Chaetoglobosin A } \\
\text { Chaetoglobosin C }\end{array}$ & Brine shrimp & $\begin{array}{l}78.3 \\
75.2 \\
83.4 \\
75.3\end{array}$ & $\begin{array}{l}\text { Qin et al., } 2009 \\
\text { (\% mortality rate at } \\
10 \mu \mathrm{g} / \mathrm{mL})\end{array}$ \\
\hline 15 & Liculaspinosa & Xylaria sp. & $\begin{array}{l}\text { Eremophilanolide } 1 \\
\text { Eremophilanolide } 2 \\
\text { Eremophilanolide } 3\end{array}$ & $\begin{array}{l}\mathrm{KB} \\
\mathrm{MCF} 7 \\
\mathrm{NCl}-\mathrm{H} 187\end{array}$ & $3.8-21$ & $\begin{array}{l}\text { Isaka et al., } 2010 \\
I_{50}(\mu \mathrm{M})\end{array}$ \\
\hline 16 & Musa acuminata & Phomopsis sp. & Oblongolides Z & $\begin{array}{l}\mathrm{KB} \\
\mathrm{BC} \\
\mathrm{NCl}-\mathrm{H} 187 \\
\text { Vero }\end{array}$ & $\begin{array}{l}37 \\
26 \\
32 \\
60\end{array}$ & $\begin{array}{l}\text { Bunyapaiboonsri } \\
\text { et al., } 2010 \\
\mathrm{IC}_{50}(\mu \mathrm{M})\end{array}$ \\
\hline 19 & Rehmannia glutinosa & Massrison sp. & Massarigenin D & $\begin{array}{l}\text { LO2 } \\
\text { HepG2 } \\
\text { MCF7 } \\
\text { A549 }\end{array}$ & $\begin{array}{l}19.6 \\
20.8 \\
11.2 \\
14.4\end{array}$ & $\begin{array}{l}\text { Sun et al., } 2011 \\
I_{50}(\mu \mathrm{g} / \mathrm{mL})\end{array}$ \\
\hline & & & Spiromassaritone & $\begin{array}{l}\text { LO2 } \\
\text { HepG2 } \\
\text { MCF7 } \\
\text { A549 }\end{array}$ & $\begin{array}{l}7.2 \\
5.6 \\
6.8 \\
9.8\end{array}$ & \\
\hline & & & Paecilospirone & $\begin{array}{l}\text { LO2 } \\
\text { HepG2 } \\
\text { MCF7 } \\
\text { A549 }\end{array}$ & $\begin{array}{c}12.4 \\
10.4 \\
7.6 \\
6.8\end{array}$ & \\
\hline 20 & Cinnamomum kanehirae & Fusarium oxysporum & Beauvericin & $\begin{array}{l}\text { PC-3 } \\
\text { Panc-1 } \\
\text { A549 }\end{array}$ & $\begin{array}{c}49.5 \\
47.2 \\
10.4\end{array}$ & $\begin{array}{l}\text { Wang et al., } 2011 \\
\text { IC }_{50}(\mu \mathrm{M})\end{array}$ \\
\hline
\end{tabular}




\section{TABLE 4 | Continued}

\begin{tabular}{|c|c|c|c|c|c|c|}
\hline $\begin{array}{l}\text { SI } \\
\text { No }\end{array}$ & Host plant & Fungal endophyte & $\begin{array}{l}\text { Isolated cytotoxic } \\
\text { compound/s }\end{array}$ & Tested cell line/s & Cytotoxicity & $\begin{array}{l}\text { References \& } \\
\text { Units of } \\
\text { cytotoxicity }\end{array}$ \\
\hline & & & (3S)-7-hydroxymellein & $\begin{array}{l}\text { MCF7 } \\
\text { NCl-H460 } \\
\text { SF-268 }\end{array}$ & $\begin{array}{l}2.78 \\
3.17 \\
2.38\end{array}$ & \\
\hline 23 & $\begin{array}{l}\text { Erythrophleum fordii } \\
\text { Oliver }\end{array}$ & Alternaria tenuissima & Tenuissimasatin & HCT-8 & $>1$ & $\begin{array}{l}\text { Fang et al., } 2012 \\
I_{50}(\mu \mathrm{mol} / \mathrm{L})\end{array}$ \\
\hline 24 & Curcuma wenyujin & $\begin{array}{l}\text { Chaetomium globosum } \\
\text { Kunze }\end{array}$ & Chaetoglobosin X & $\begin{array}{l}\mathrm{H} 22 \\
\text { MFC }\end{array}$ & $\begin{array}{r}3.125 \\
6.25\end{array}$ & $\begin{array}{l}\text { Wang et al., } 2012 \\
I_{50}(\mu \mathrm{g} / \mathrm{mL})\end{array}$ \\
\hline 25 & Erythrina variegata & Alternaria sp. & $\begin{array}{l}\text { Altersolanol } \\
\text { Macrosporin } \\
\text { 1,2,4,5-tetrahydroxy-7- } \\
\text { methoxy-2-methyl-1,2,3,4- } \\
\text { tetrahydroanthracene-9,10- } \\
\text { dione }\end{array}$ & Angiogenesis assays & - & $\begin{array}{l}\text { Pompeng et al., } \\
2013\end{array}$ \\
\hline 29 & Ipomoea batatas & Aspergillus glaucus & $\begin{array}{l}\text { 2, } \\
\text { 14-dihydrox-7-drimen-12, } \\
\text { 11-olide }\end{array}$ & $\begin{array}{l}\text { HepG2 } \\
\text { MCF7 }\end{array}$ & $\begin{array}{c}61 \\
41.7\end{array}$ & $\begin{array}{l}\text { Mohamed et al., } \\
2013 \\
\mathrm{IC}_{50}(\mu \mathrm{g} / \mathrm{mL})\end{array}$ \\
\hline 30 & Gloriosa superba & Aspergillus sp. & Colchatetralene & $\begin{array}{l}\text { THP-1 } \\
\text { MCF7 }\end{array}$ & $\begin{array}{l}30 \\
50\end{array}$ & $\begin{array}{l}\text { Budhiraja et al., } \\
2013 \\
{ }^{1 C_{50}}(\mu \mathrm{g} / \mathrm{mL})\end{array}$ \\
\hline 31 & Panax ginseng & Penicillium melinii & Ginsenocin & $\begin{array}{l}\text { MKN45 } \\
\text { LOVO } \\
\text { A549 } \\
\text { MDA-MB-435 } \\
\text { HepG2 } \\
\text { HL-60 }\end{array}$ & $\begin{array}{l}1.91 \\
2.20 \\
5.03 \\
1.39 \\
2.34 \\
0.49\end{array}$ & $\begin{array}{l}\text { Zheng et al., } 2013 \\
\text { IC }_{50}(\mu \mathrm{g} / \mathrm{mL})\end{array}$ \\
\hline
\end{tabular}


TABLE 4 | Continued

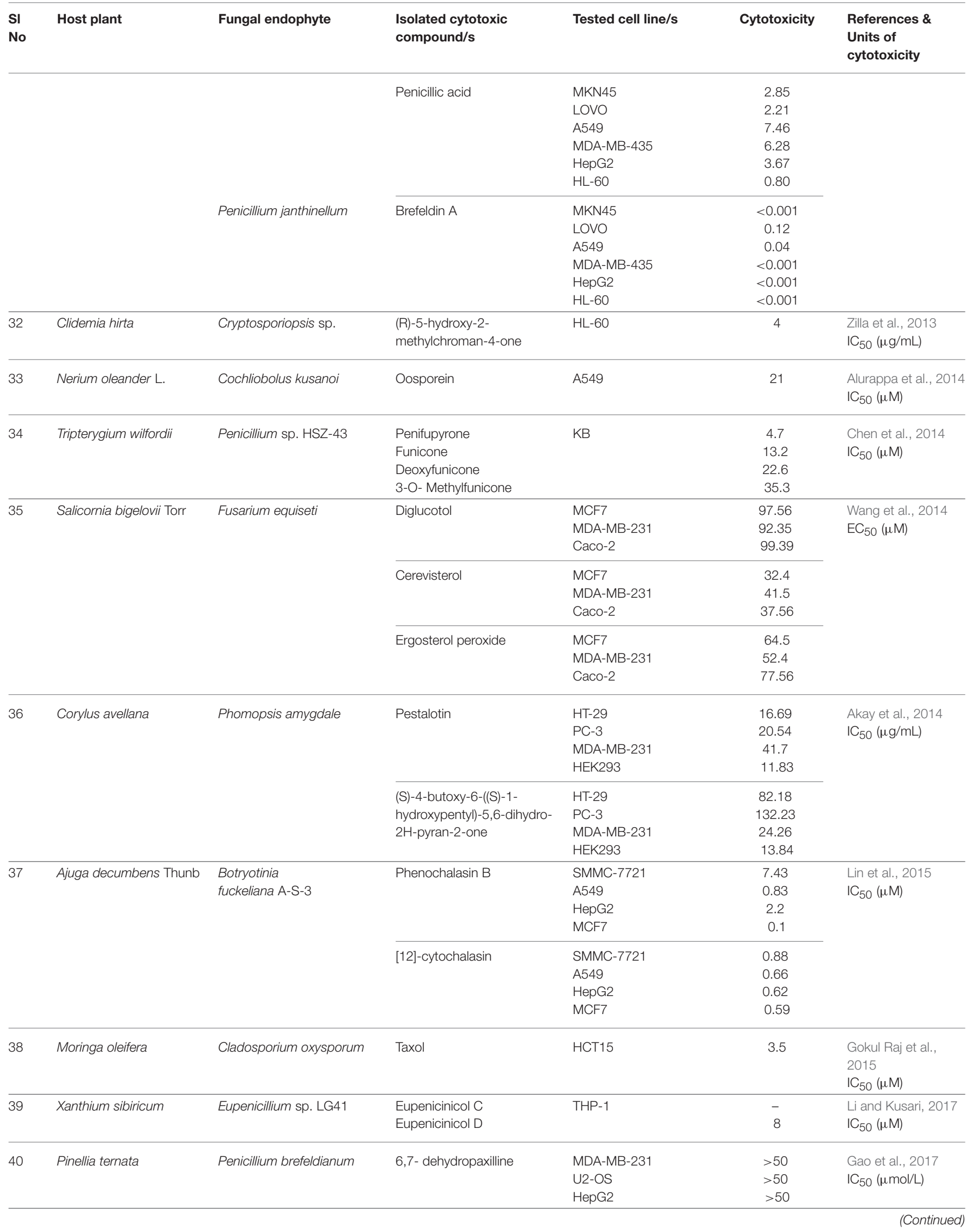


TABLE 4 | Continued

\begin{tabular}{|c|c|c|c|c|c|c|}
\hline $\begin{array}{l}\text { SI } \\
\text { No }\end{array}$ & Host plant & Fungal endophyte & $\begin{array}{l}\text { Isolated cytotoxic } \\
\text { compound/s }\end{array}$ & Tested cell line/s & Cytotoxicity & $\begin{array}{l}\text { References \& } \\
\text { Units of } \\
\text { cytotoxicity }\end{array}$ \\
\hline & & & Spirotryprostatin F & $\begin{array}{l}\text { MDA-MB-231 } \\
\text { U2-OS } \\
\text { HepG2 }\end{array}$ & $\begin{array}{l}35.5 \\
>50 \\
14.1\end{array}$ & \\
\hline & & & $\mathrm{N}$-demethyl melearoride A & $\begin{array}{l}\text { MDA-MB-231 } \\
\text { U2-OS } \\
\text { HepG2 }\end{array}$ & $\begin{array}{l}>50 \\
>50 \\
36.6\end{array}$ & \\
\hline
\end{tabular}

Among the isolated compounds, only potent compounds are included in the column "isolated compound/s".

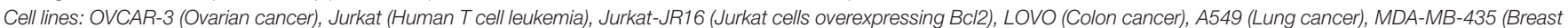

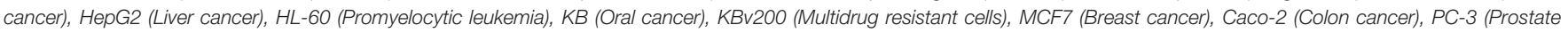
cancer), HeLa (Cervical cancer), A431 (Skin cancer), MDA-MB-231 (Breast cancer), MIA PaCa-2 (Pancreatic cancer), T47D (Breast cancer), HT 29 (Colon cancer), HEK293 (Kidney),

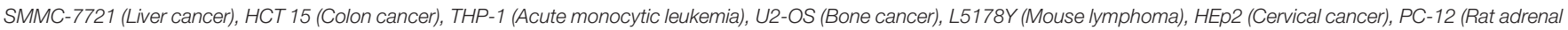

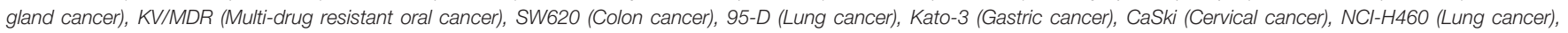

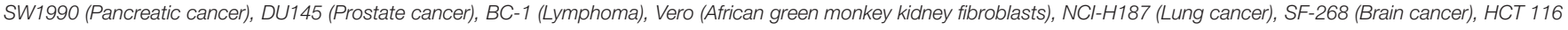

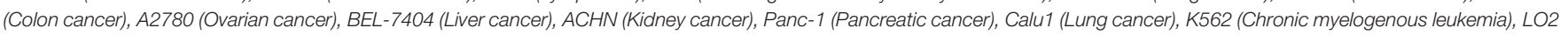

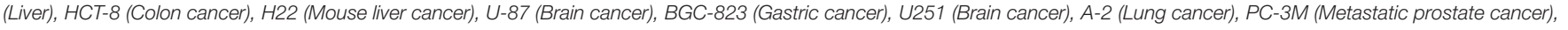

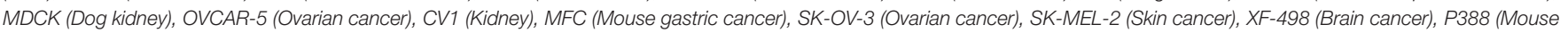
lymphoma), MKN45 (Gastric cancer), and U937 (Histiocytic lymphoma).

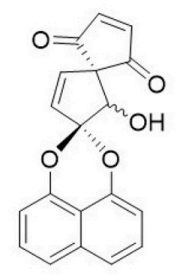

17: Spiropreussione A 18: Ent-4(15)-eudesmen-11-ol-1-one

The endophyte Phomopsis sp. was isolated from the leaf of Musa acuminata collected at Doi Suthep Pui National Park, Chiang Mai Province, Thailand. The fungus produced six new oblongolides and seven known compounds. Among the new compounds, oblongolides $Y$ (22) and $Z$ (23) were cytotoxic against the tested cells. The compound 22 was superior over its counterpart with the $\mathrm{IC}_{50}$ values of 37 , 26, 32, and $60 \mu \mathrm{M}$ against $\mathrm{KB}, \mathrm{BC}$ and NCI-H187, and Vero cell lines, respectively. The positive control, doxorubicin had the $\mathrm{IC}_{50}$ values of $0.24 \mu \mathrm{M}$ for $\mathrm{KB}, 0.30 \mu \mathrm{M}$ for $\mathrm{BC}$, and $0.08 \mu \mathrm{M}$ for NCI-H187 (Bunyapaiboonsri et al., 2010). In the next study, the fungus was found to produce four new compounds named oblongolides $\mathrm{C} 1, \mathrm{P} 1, \mathrm{X} 1$, and 6hydroxyphomodiol together with eight known compounds including oblongolides $\mathrm{B}, \mathrm{C}, \mathrm{D}, \mathrm{O}, \mathrm{P}, \mathrm{U},(3 \mathrm{R}, 4 \mathrm{aR}, 5 \mathrm{~S}, 6 \mathrm{R})$ 6-hydroxy-5-methylramulosin, and (3R)-5-methylmellein. The new compounds exhibited weak selective cytotoxicity against HepG2 cells with an inhibitory rate ranging between 16.89 and 28.59\% (Lin et al., 2011). An endophytic Alternaria sp., associated with the Egyptian medicinal plant Polygonum senegalense was<smiles>C=C1C(=O)O[C@H]2C[C@]3(O)[C@@H](OC)CCC(C)[C@]3(C)C[C@]12O</smiles>

20: Eremophilanolide 2 21: Eremophilanolide 3

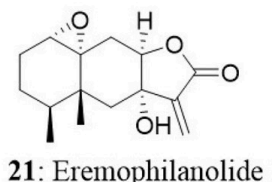

isolated. When the endophyte was cultured on the solid rice medium, four new compounds (3'-hydroxyalternariol 5-Omethyl ether, desmethylaltenusin, alterlactone, and alternaric acid) and five known compounds [alternariol (24), alternariol 5-O-methyl ether, altenusin, talaroflavone, and altenuene) were obtained. And when the fungus was grown on liquid Wickerham medium, two new compounds (alternariol 5-Osulfate (25) and alternariol 5-O-methyl ether (26)] and six known compounds (alternariol, alternariol 5-O-methyl ether, altenusin, 2,5-dimethyl-7-hydroxychromone, tenuazonic acid, and altertoxin I) were isolated. Overall, fungal extract analysis revealed the presence of 15 natural compounds, out of which seven were new compounds. Compounds 24, 25, and 26 showed cytotoxicity against L5178Y cells with $\mathrm{EC}_{50}$ values ranging between 1.7 and $7.8 \mu \mathrm{g} / \mathrm{mL}$, compared with the positive control (kahalalide F), which had the $\mathrm{EC}_{50}$ value of $6.3 \mu \mathrm{g} / \mathrm{mL}$. The two bicyclic acid derivatives, Alutenusin (27) and desmethylaltenusin (28) exhibited moderate cytotoxic activity against L5178Y cells, with $\mathrm{EC}_{50}$ values of 6.8 and $6.2 \mu \mathrm{g} / \mathrm{mL}$, respectively (Aly et al., 2008).
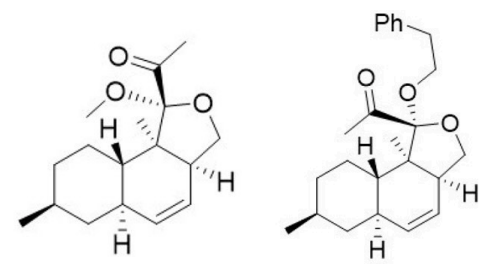

22: Oblongolide Y 23: Oblongolide $Z$<smiles>Cc1cc(O)cc2oc(=O)c3c(O)cc(O)cc3c12</smiles>

24: Alternariol<smiles>Cc1cc(O)cc2oc(=O)c3c(O)cc(OS(=O)(=O)O)cc3c12</smiles><smiles>COc1cc(O)c2c(=O)oc3cc(O)cc(C)c3c2c1</smiles>

25: Alternariol-5-O-Sulfate 26: Alternariol-5-O-methyl ester 
TABLE 5 | List of anticancer compounds isolated from endophytic fungi from mangrove habitats.

\begin{tabular}{|c|c|c|c|c|c|c|}
\hline SI No & Host plant & Fungal endophyte & $\begin{array}{l}\text { Isolated cytotoxic } \\
\text { compound/s }\end{array}$ & Tested cell line/s & Cytotoxicity & $\begin{array}{l}\text { References \& Units } \\
\text { of cytotoxicity }\end{array}$ \\
\hline 1 & Excoecaria agallocha & $\begin{array}{l}\text { Phomopsis sp. } \\
\text { ZSU-H76 }\end{array}$ & $\begin{array}{l}\text { 2-(7'-hydroxyoxooctyl)- } \\
\text { 3-hydroxy-5- } \\
\text { methoxybenzeneacetic } \\
\text { acid ethyl ester }\end{array}$ & $\begin{array}{l}\text { HEp2 } \\
\text { HepG2 }\end{array}$ & $\begin{array}{l}25 \\
30\end{array}$ & $\begin{array}{l}\text { Huang et al., } 2009 \\
\mathrm{IC}_{50}(\mu \mathrm{g} / \mathrm{mL})\end{array}$ \\
\hline 2 & Rhizophora mucronata & Pestalotiopsis sp. & $\begin{array}{l}\text { Cytosporones J-N } \\
\text { Pestalasins A-E } \\
\text { Pestalotiopsoid A }\end{array}$ & $\begin{array}{l}\text { L5178Y } \\
\text { HeLa } \\
\text { PC12 }\end{array}$ & $\begin{array}{l}\text { Not Active up to } \\
10 \mu \mathrm{g} / \mathrm{mL}\end{array}$ & Xu et al., 2009a \\
\hline 3 & Rhizophora mucronata & Pestalotiopsis sp. & $\begin{array}{l}\text { Pestalotiopsone A } \\
\text { Pestalotiopsone B } \\
\text { Pestalotiopsone C } \\
\text { Pestalotiopsone D } \\
\text { Pestalotiopsone E } \\
\text { Pestalotiopsone F }\end{array}$ & L5178Y & $\begin{array}{l}\text { NA } \\
\text { NA } \\
\text { NA } \\
\text { NA } \\
\text { NA } \\
26.89\end{array}$ & $\begin{array}{l}\text { Xu et al., 2009a } \\
I_{50}(\mu \mathrm{M})\end{array}$ \\
\hline 4 & Not mentioned & $\begin{array}{l}\text { Mangrove endophytic } \\
\text { fungus No. ZSU44 }\end{array}$ & Secalonic acid D & $\begin{array}{l}\text { HL60 } \\
\text { K562 }\end{array}$ & $\begin{array}{l}0.38 \\
0.43\end{array}$ & $\begin{array}{l}\text { Zhang J. Y. et al., } 2009 \\
I_{50}(\mu \mathrm{M})\end{array}$ \\
\hline 5 & Excoecaria agallocha & Phomopsis sp. & $\begin{array}{l}\text { Phomopsis-H76 A } \\
\text { Phomopsis-H76 B } \\
\text { Phomopsis-H76 C }\end{array}$ & $\begin{array}{l}\text { KB } \\
\text { KBv200 } \\
\text { MCF7 }\end{array}$ & $\begin{array}{l}\text { All the compounds are } \\
\text { inactive against all the } \\
\text { tested cell lines }\end{array}$ & Yang et al., 2010 \\
\hline 6 & Kandelia woody tissue & $\begin{array}{l}\text { Halorosellinia sp. } \\
\text { Guignardia sp. }\end{array}$ & $\begin{array}{l}\text { 1-hydroxy-3-methyl } \\
\text { anthracene-9,10-dione }\end{array}$ & $\begin{array}{l}\text { KB } \\
\text { KBv200 }\end{array}$ & $\begin{array}{l}3.17 \\
3.21\end{array}$ & $\begin{array}{l}\text { Zhang J. Y. et al., } 2010 \\
I_{50}(\mu \mathrm{M})\end{array}$ \\
\hline 7 & Sonneratia apetala & Zh6-B1 (unidentified) & $\begin{array}{l}\text { 3R,5R-Sonnerlactone } \\
\text { 3R,5S-Sonnerlactone }\end{array}$ & KV/MDR & $\begin{array}{l}42.4 \\
41.6\end{array}$ & $\begin{array}{l}\text { Li et al., } 2010 \\
\% \text { inhibition at } 100 \mu \mathrm{M}\end{array}$ \\
\hline \multirow[t]{3}{*}{8} & Xylocarpus granatum & XG8D (unidentified) & Merulin A & $\begin{array}{l}\text { BT474 } \\
\text { SW620 }\end{array}$ & $\begin{array}{l}4.98 \\
4.84\end{array}$ & $\begin{array}{l}\text { Chokpaiboon et al., } \\
2010 \\
\mathrm{IC}_{50}(\mu \mathrm{g} / \mathrm{mL})\end{array}$ \\
\hline & & & Merulin B & $\begin{array}{l}\text { BT474 } \\
\text { SW620 }\end{array}$ & $\begin{array}{l}>10 \\
>10\end{array}$ & \\
\hline & & & Merulin C & $\begin{array}{l}\text { BT474 } \\
\text { SW620 }\end{array}$ & $\begin{array}{l}1.57 \\
4.11\end{array}$ & \\
\hline 9 & Acanthus ilicifolius & Penicillium sp. & Penicinoline & $\begin{array}{l}\text { 95-D } \\
\text { HepG2 } \\
\text { HeLa } \\
\text { KB } \\
\text { KBv200 } \\
\text { HEp2 }\end{array}$ & $\begin{array}{l}0.57 \\
6.5 \\
>100 \\
>100 \\
>100 \\
>100\end{array}$ & $\begin{array}{l}\text { Shao et al., } 2010 \\
\mathrm{IC}_{50}(\mu \mathrm{g} / \mathrm{mL})\end{array}$ \\
\hline 10 & $\begin{array}{l}\text { Unidentified mangrove } \\
\text { (Taiwan Strait) }\end{array}$ & Paecilomyces sp. & $\begin{array}{l}\text { Paeciloxocins A } \\
\text { Paeciloxocins B }\end{array}$ & HepG2 & $\begin{array}{l}1 \\
65\end{array}$ & $\begin{array}{l}\text { Wen et al., } 2010 \\
I_{50}(\mu \mathrm{g} / \mathrm{mL})\end{array}$ \\
\hline \multirow[t]{2}{*}{11} & Excoecaria agallocha & Penicillium expansum & Expansols A & $\begin{array}{l}\text { A549 } \\
\text { HL-60 }\end{array}$ & $\begin{array}{l}\text { NR } \\
15.7\end{array}$ & $\begin{array}{l}\text { Lu et al., } 2010 \\
I_{50}(\mu \mathrm{M})\end{array}$ \\
\hline & & & Expansols B & $\begin{array}{l}\text { A549 } \\
\text { HL-60 }\end{array}$ & $\begin{array}{l}1.9 \\
5.4\end{array}$ & \\
\hline 12 & Kandelia candel & Fusarium sp. & $\begin{array}{l}\text { 5-O-methyl-2' - } \\
\text { methoxy-3'- } \\
\text { methylalpinumisoflavone }\end{array}$ & $\begin{array}{l}\text { HEp2 } \\
\text { HepG2 }\end{array}$ & $\begin{array}{l}4 \\
11\end{array}$ & $\begin{array}{l}\text { Huang et al., } 2010 \\
(\mu \mathrm{mol} / \mathrm{mL})\end{array}$ \\
\hline 13 & Aegiceras corniculatum & Alternaria sp. ZJ9-6B & Alterporriol K & $\begin{array}{l}\text { MDA-MB-435 } \\
\text { MCF-7 }\end{array}$ & $\begin{array}{l}26.97 \\
29.11\end{array}$ & $\begin{array}{l}\text { Huang et al., } 2011 \\
\mathrm{IC}_{50}(\mu \mathrm{M})\end{array}$ \\
\hline
\end{tabular}


TABLE 5 | Continued

\begin{tabular}{|c|c|c|c|c|c|c|}
\hline SI No & Host plant & Fungal endophyte & $\begin{array}{l}\text { Isolated cytotoxic } \\
\text { compound/s }\end{array}$ & Tested cell line/s & Cytotoxicity & $\begin{array}{l}\text { References \& Units } \\
\text { of cytotoxicity }\end{array}$ \\
\hline & & & Alterporriol L & $\begin{array}{l}\text { MDA-MB-435 } \\
\text { MCF7 }\end{array}$ & $\begin{array}{l}13.11 \\
20.04\end{array}$ & \\
\hline & & & Alterporriol M & $\begin{array}{l}\text { MDA-MB-435 } \\
\text { MCF-7 }\end{array}$ & $\begin{array}{l}\text { NT } \\
\text { NT }\end{array}$ & \\
\hline 15 & $\begin{array}{l}\text { Rhizophora } \\
\text { annamalayan }\end{array}$ & Fusarium oxysporum & Taxol & NT & NT & Elavarasi et al., 2012 \\
\hline \multirow{3}{*}{16} & & & Rhytidchromones B & $\begin{array}{l}\text { MCF7 } \\
\text { HepG2 } \\
\text { Kato-3 } \\
\text { CaSki }\end{array}$ & $\begin{array}{l}>25 \\
>25 \\
21.4 \\
>25\end{array}$ & \\
\hline & & & Rhytidchromones C & $\begin{array}{l}\text { MCF7 } \\
\text { HepG2 } \\
\text { Kato-3 } \\
\text { CaSki }\end{array}$ & $\begin{array}{l}>25 \\
>25 \\
>25 \\
>25\end{array}$ & \\
\hline & & & Rhytidchromones D & $\begin{array}{l}\text { MCF7 } \\
\text { HepG2 } \\
\text { Kato-3 } \\
\text { CaSki }\end{array}$ & $\begin{array}{l}>25 \\
>25 \\
16.8 \\
>25\end{array}$ & \\
\hline
\end{tabular}

Among the isolated compounds, only potent compounds are included in the column "isolated compound/s".

NA, Not active; NR, Not reported; NT, Not tested.

Ergoflavin (29) belongs to the class of compounds called ergochromes, and it was first isolated from the ergot fungus (Claviceps purpurea) followed by identification in Phoma terrestris, Pyrenochaeta terrestris, Penicillium oxalicum, and Aspergillus sp. The ergoflavin was isolated from endophyte collected from the leaf of Mimusops elengi. The isolated ergoflavin inhibited human TNF- $\alpha$ and IL- 6 with IC $_{50}$ values of 1.9 and $1.2 \mu \mathrm{M}$, respectively. Further investigation revealed that ergoflavin induces cytotoxicity in ACHN, NCI-H460, Panc-1, HCT116, and Calu1 cell lines with $\mathrm{IC}_{50}$ values of 1.2, 4.0, 2.4, 8.0, and 1.5 $\mu \mathrm{M}$, respectively (Deshmukh et al., 2009).
The endophytic fungus Massrison sp. was isolated from the roots of wild Rehmannia glutinosa collected from Wushe County, Henan Province, China. Extract from the culture broth revealed the presence of massarigenin D (30), spiromassaritone (31), and paecilospirone (32). These compounds contain a rare spiro5,6-lactone ring skeleton. The cytotoxic effects of these three compounds were tested against LO2, HepG2, MCF7, and A-2 cells. The compounds showed good cytotoxicity against the tested cells. Spiromassaritone displayed potent cytotoxicity against all the tested cells with an $\mathrm{IC}_{50}$ value ranging between 5.6 and $9.8 \mu \mathrm{g} / \mathrm{mL}$ (Sun et al., 2011).<smiles>COc1cc(O)c(C(=O)O)c(-c2cc(O)c(O)cc2C)c1</smiles>

27: Alutenusin<smiles>Cc1cc(O)c(O)cc1-c1cc(O)cc(O)c1C(=O)O</smiles>

28: Desmethylalutenusin

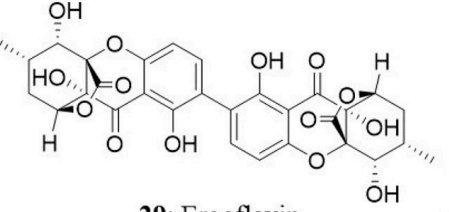

29: Ergoflavin
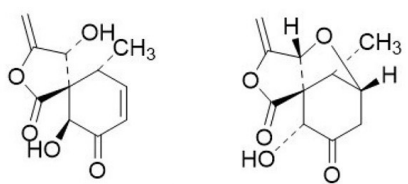

30: Massarigenin D 31: Spiromassaritone 32: Paecilospirone

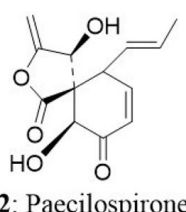


TABLE 6 | List of marine cytotoxic endophytic fungal metabolites.

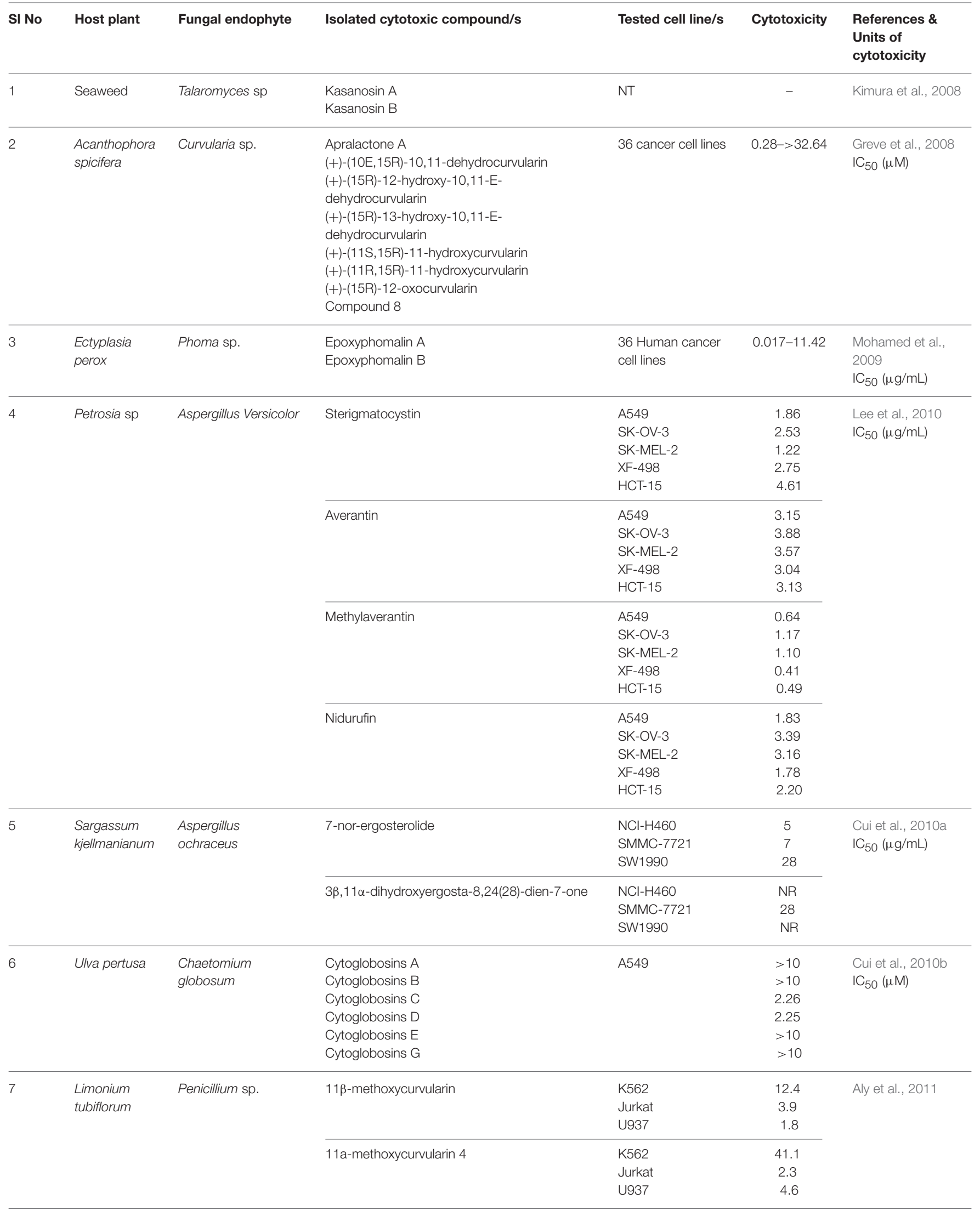


TABLE 6 | Continued

\begin{tabular}{|c|c|c|c|c|c|c|}
\hline SI No & Host plant & Fungal endophyte & Isolated cytotoxic compound/s & Tested cell line/s & Cytotoxicity & $\begin{array}{l}\text { References \& } \\
\text { Units of } \\
\text { cytotoxicity }\end{array}$ \\
\hline & & & Dehydrocurvularin 6 & $\begin{array}{l}\text { K562 } \\
\text { Jurkat } \\
\text { U937 }\end{array}$ & $\begin{array}{c}37.0 \\
5.5 \\
2.5\end{array}$ & \\
\hline & & & $\begin{array}{l}\text { 1-chloro-2,4-dihydroxy-5-methoxy-7- } \\
\text { methylanthraquinone }\end{array}$ & $\begin{array}{l}\text { K562 } \\
\text { Jurkat } \\
\text { U937 }\end{array}$ & $\begin{array}{c}24.8 \\
13.3 \\
7.6\end{array}$ & \\
\hline
\end{tabular}

Among the isolated compounds, only potent compounds are included in the column "isolated compound/s".

NA, Not active; NR, Not reported; NT, Not tested.

The endophytic fungus was collected from stems of Salvia officinalis from the mountain of Beni-Mellal, Morocco. Chemical analysis of secondary metabolites revealed the presence of two known compounds namely, cochliodinol (33) and isocochliodinol (34). The bioactive compounds were tested for cytotoxicity against L5178Y cells. Cochliodinol was potent than isocochliodinol, with an $\mathrm{EC}_{50}$ of $7.0 \mu \mathrm{g} / \mathrm{mL}$ compared with $71.5 \mu \mathrm{g} / \mathrm{mL}$ for isocochliodinol. The authors interpreted that, the difference in cytotoxicity of structurally related compounds was due to the position of prenyl substituents at the indole rings (Debbab et al., 2009b).<smiles>CC(C)=CCc1ccc2c(C3=C(O)C(=O)C(c4c[nH]c5cc(CC=C(C)C)ccc45)=C(O)C3=O)c[nH]c2c1</smiles>

The fungal endophyte Pestalotiopsis fici was isolated from branches of Camellia sinensis in the suburb of Hangzhou, Zhejiang province, China. Seven new isoprenylated chromone derivatives namely pestaloficiols F-L were obtained. Among the newly isolated compounds, pestaloficiol J (35), K (36), L (37) exhibited cytotoxicity with $\mathrm{IC}_{50}$ values ranging between 8.7 and 99.3 $\mu \mathrm{M}$ for HeLa cell line. The $\mathrm{IC}_{50}$ values of other compounds ranged between $8.7 \mu \mathrm{M}$ and $>136.1 \mu \mathrm{M}$ for HeLa cells and 17.4 $\mu \mathrm{M}$ and $>153.8 \mu \mathrm{M}$ for MCF7 cells. 5-fluorouracil was used as a positive control with $\mathrm{IC}_{50}$ values of 10 and $15 \mu \mathrm{M}$ against HeLa and MCF7 respectively (Liu L. et al., 2009).<smiles>CC(C)=CCc1cc(O)cc2c1OC(C)(C)CC2=O</smiles>

35: Pestaloficiol J<smiles>CC(=O)OC1=CC(C)(C)Oc2c(CC=C(C)C)cc(O)cc21</smiles>

36: Pestaloficiol K<smiles>COC(=O)c1cc(OC)cc(O)c1C(=O)c1c(O)cc(C)c(C2=CC(C)(C)Oc3c(CC=C(C)C)cc(O)cc32)c1O</smiles>

37: Pestaloficiol L
Six novel benzofuranone-derived $\gamma$-lactones called photinides A-F (38-43), were isolated from the endophyte Pestalotiopsis photiniae. The endophyte was isolated from Roystonea regia collected from Jianfeng mountain, Hainan province, China. Photinides A-F exhibited modest and selective cytotoxicity against MDA-MB-231 cells with an inhibitory rate of 24.4, $24.2,23.1,24.4$, and $24.6 \%$, respectively, at a concentration of $10 \mu \mathrm{g} / \mathrm{mL}$. No cytotoxicity was observed at $10 \mu \mathrm{g} / \mathrm{mL}$ against HeLa cells (Ding et al., 2009).<smiles>COc1cccc2c1C(=O)/C(=C(/C)[C@H]1OC(=O)C[C@H]1C)O2</smiles><smiles>COc1cccc2c1C(=O)C(=C(C)C1OC(=O)C[C@H]1C)O2</smiles>

39: Photinide B

38: Photinide A<smiles>CC1CC(=O)O[C@H]1/C(CO)=C1/Oc2cccc(O)c2C1=O</smiles>

41: Photinide D<smiles>COc1cccc2c1C(=O)[C@]1(O2)O[C@H]1[C@@H]1OC(=O)C[C@H]1C</smiles>

42: Photinide $\mathrm{E}$<smiles>C/C(=C1\Oc2cccc(O)c2C1=O)[C@H]1OC(=O)C[C@H]1C</smiles>

40: Photinide C<smiles>COc1cccc2c1C(=O)[C@@H]([C@](C)(O)[C@H]1OC(=O)C[C@H]1C)O2</smiles>

43: Photinide F 
The endophyte Stemphylium globuliferum was isolated from the stem tissues of Egyptian medicinal plant Mentha pulegium collected in Morocco. Chemical investigation of extract revealed the presence of five novel compounds (alterporriol $\mathrm{G}$, atropisomer alterporriol $\mathrm{H}$, altersolanol $\mathrm{K}$, altersolanol $\mathrm{L}$, and stemphypyrone) and eight known compounds (6-Omethylalaternin, macrosporin, altersolanol A, alterporriol E, alterporriol D, alterporriol A, alterporriol B, and altersolanol J). The isolated compounds were tested for cytotoxic activity against L5178Y cells. An unresolved mixture of alterporriol $\mathrm{G}$ (44) and its atropisomer alterporriol $\mathrm{H}$ (45) exhibited the most potent cytotoxicity with an $\mathrm{EC}_{50}$ value of $2.7 \mu \mathrm{g} / \mathrm{mL}$. The known compound 6-O-methylalaternin (46) also exhibited good cytotoxicity with an $\mathrm{EC}_{50}$ value of $4.2 \mu \mathrm{g} / \mathrm{mL}$. The positive control (kahalalide F) exhibited an $\mathrm{EC}_{50}$ value of $6.3 \mu \mathrm{g} / \mathrm{mL}$ (Debbab et al., 2009a).<smiles>COc1cc2c(c(O)c1-c1c(OC)cc(O)c3c1C(=O)C1=C(C3=O)C(O)[C@@](C)(O)[C@H](O)C1O)C(=O)c1cc(O)c(C)cc1C2=O</smiles><smiles>COc1cc(O)c2c(c1)C(=O)c1cc(C)c(O)c(O)c1C2=O</smiles>

46: 6-O-methylalaternin

44: Alterporriol G; 45: Alterporriol $\mathrm{H}$

(Atropisomers)

The endophyte Phyllosticta spinarum isolated from Platycladus orientalis from the Sonoran desert produced five new compounds $\quad\left[(+)-(5 S, 10 S)-4^{\prime}\right.$-hydroxymethylcyclozonarone, 3-ketotauranin, 3R-hydroxytauranin, 12-hydroxytauranin, and phyllospinarone] along with a known compound tauranin (47). Tauranin exhibited cytotoxicity against NCI-H460, MCF7, SF-268, PC-3M, and MIA PaCa-2 cells with $\mathrm{IC}_{50}$ values of $4.3,1.5,1.8,3.5$, and $2.8 \mu \mathrm{M}$, respectively (Wijeratne et al., 2008). A newly modified dipeptide trichodermamide C (48) was isolated from the endophytic fungus Eupenicillium sp., from the rainforest tree Glochidion ferdinandi (Euphorbiaceae) in Australia. Trichodermamide $\mathrm{C}$ displayed cytotoxicity toward HCT116 and A549 cell lines with $\mathrm{IC}_{50}$ values of 0.68 and $4.28 \mu \mathrm{g} / \mathrm{mL}$, respectively (Davis et al., 2008).<smiles>C=C1CC[C@H]2C(C)(C)CCC[C@]2(C)[C@H]1CC1=C(O)C(=O)C(CO)=CC1=O</smiles>

47: Tauranin

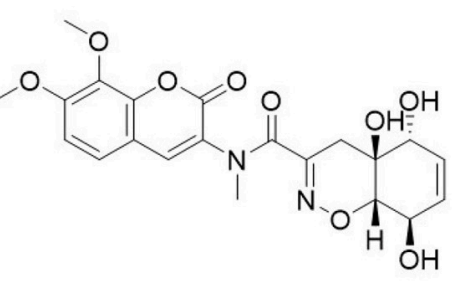

48: Trichodermamide $\mathrm{C}$
In another study, a new dihydrobenzofuran-2,4-dione derivative named as annulosquamulin (49) along with 10 known compounds were obtained from the endophytic fungus Annulohypoxylon squamulosum. The endophyte was isolated from the stem bark of the medicinal plant Cinnamomum sp. at Fu-Shan Botanical Garden, I-lan County, Taiwan. Annulosquamulin was evaluated for its cytotoxicity against MCF7, NCI-H460, and SF-268 cell lines. The $\mathrm{IC}_{50}$ values of annulosquamulin were $3.19,3.38$, and $2.46 \mu \mathrm{g} / \mathrm{mL}$, respectively (Cheng et al., 2012).<smiles>CCCCCCCCCC(O)[C@H]1C(=O)O[C@]2(C)C(=O)C3=C(COC3=O)C[C@H]12</smiles>

\section{9: Annulosquamulin}

The endophytic fungal strain Allantophomopsis lycopodina was isolated from a tree branch on the Gassan stock farm, Yamagata, Japan, yielding a new natural product, allantopyrone A (50), and a previously reported islandic acid-II methyl ester (51). Both compounds exhibited good cytotoxic activity against HL60 cells with $\mathrm{IC}_{50}$ values of 0.32 and $6.55 \mu \mathrm{M}$, respectively. Cleavage of the genomic DNA is a hallmark event in the cells committed to apoptosis (Sebastian et al., 2016) which leads to the formation of cells with lesser DNA content called hypodiploid cells (Mohan et al., 2014, 2016; Roopashree et al., 2015). Treatment of HL60 cells with these compounds resulted in fragmentation of genomic DNA and N-acetyl-L-cysteine prevented this effect. Allantopyrone A demonstrated stronger cytotoxicity and apoptosis-inducing activity than the islandic acid-II methyl ester (Shiono et al., 2010).<smiles>C/C=C\C=C/C(=O)OCc1c(OC)c(CO)c(/C=C/CO)oc1=O</smiles>

50: Allantopyrone A<smiles>C=C(/C=C/c1oc(=O)c(CO)c(OC)c1CO)OC</smiles>

51: Islandic acid II methyl ester

An endophytic fungus, Cochliobolus kusanoi, isolated from the stem of Nerium oleander L. produced the known compound oosporein (52). The cytotoxicity of oosporein was evaluated toward A549 and presented the $\mathrm{IC}_{50}$ value of $21 \mu \mathrm{M}$ (Alurappa et al., 2014). The cytotoxic potential of oosporein in the MDCK cell line and its role in oxidative stress in vivo and in vitro have been previously reported by our research group (Ramesha et al., 2015).

The endophytic fungus Penicillium sp., HSZ-43 was isolated from the leaves of Chinese traditional medicinal plant Tripterygium wilfordii. The endophyte was found to 
produce a novel compound, penifupyrone (53), along with three known analogs: funicone (54), deoxyfunicone (55), and 3-Omethylfunicone (56). The isolated compounds were tested for cytotoxicity against KB cells. Penifupyrone exhibited moderate cytotoxicity with an $\mathrm{IC}_{50}$ value of $4.7 \mu \mathrm{M}$. The $\mathrm{IC}_{50}$ values of funicone, deoxyfunicone and 3-O-methylfunicone were 13.2, 22.6, and 35.3 $\mu \mathrm{M}$, respectively (Chen et al., 2014).

Meroterpenoids namely arisugacin I (57) arisugacin J (58), arisugacin $F(59)$, arisugacin $G(60)$, arisugacin $B(61)$, territrem C (62), and territrem B (63) were obtained from the endophytic fungus Penicillium sp. SXH-65. The fungus was isolated from the saline-alkaline soil along the coast of Laizhou Bay in Dongying, China. The isolated compounds were evaluated against a panel of cancer cell lines. Arisugacin B and Arisugacin F exhibited weak cytotoxicity against HeLa, HL-60, and K562 cell lines with $\mathrm{IC}_{50}$ values ranging from 24 to $60 \mu \mathrm{M}$ (Sun et al., 2014). A new sesquiterpenoid, Perenniporin A (64), from the endophytic fungus Perenniporia tephropora Z41 from T. chinensis var. mairei exhibited moderate cytotoxic activity with $\mathrm{IC}_{50}$ values ranging from 6 to $58 \mu \mathrm{g} / \mathrm{mL}$ against three human cancer cell lines (HeLa, SMMC-7721, and Panc-1). On the other hand, ergosterol (65) from the same source showed significant cytotoxicity against the HeLa, SMMC-7721, and Panc-1 cell lines with $\mathrm{IC}_{50}$ values of 1.16 , 11.63, and $11.80 \mu \mathrm{g} / \mathrm{mL}$ (Wu et al., 2013).

A novel diglucotol (66), together with five known compounds, cerebroside C (67), N-acetyltryptamine (68),<smiles>COC(=O)c1cc(OC)cc(OC)c1C(=O)c1coc2c1OC(C)CC2=O</smiles>

52: Oosporein

53: Penifupyrone<smiles>C/C=C/c1occ(C(=O)c2c(OC)cc(OC)cc2C(=O)OC)c(=O)c1OC</smiles><smiles>COc1ccc(-c2cc(O)c(C/C=C(\C)CC/C=C(\C)CCC(O)C(C)(C)O)c(=O)o2)cc1</smiles>

57: Arisugasin I<smiles>C/C=C/c1occ(C(=O)c2c(OC)cc(OC)cc2C(=O)OC)c(=O)c1O</smiles>

54: Funicone<smiles>C/C=C/c1cc(=O)c(C(=O)c2c(OC)cc(OC)cc2C(=O)OC)co1</smiles>

55: Deoxyfunicone

56: 3-O- methylfunicone<smiles>COc1ccc(-c2cc3c(c(=O)o2)C[C@@]2(C)C(C)(C)CCC[C@@]2(C)O3)cc1</smiles>

58: Arisugasin J

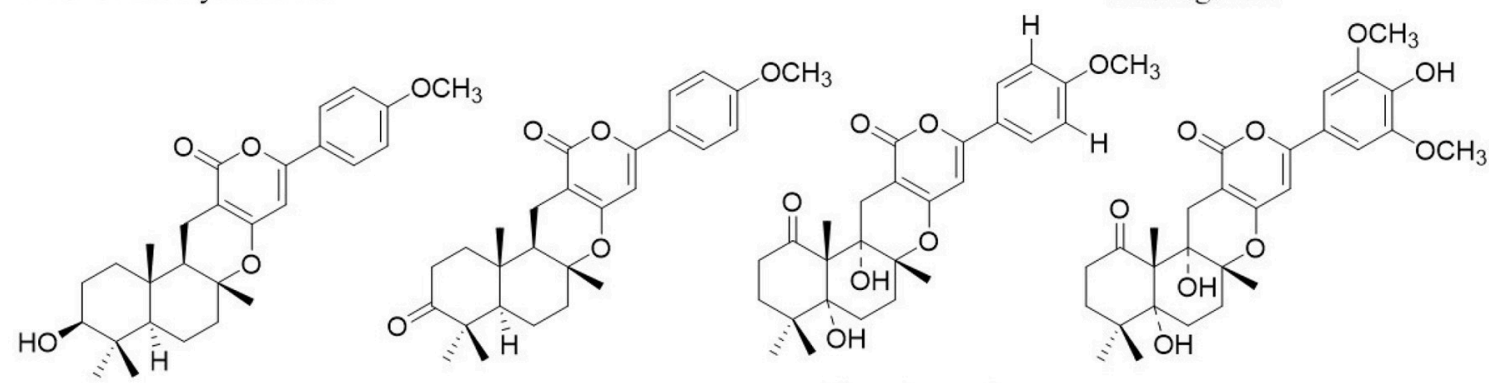

59: Arisugasin F

60: Arisugasin G

61: Arisugasin B

62: Territrem C

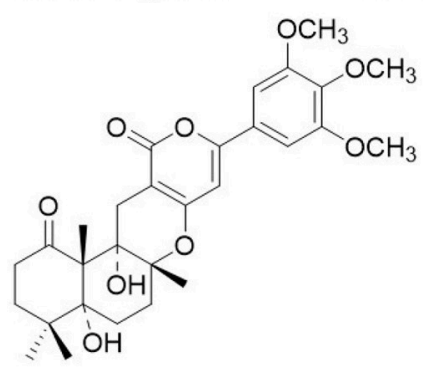

63: Territrem B<smiles>CC(C)(O)[C@@H](O)c1cc(C2CC=C(C(=O)O)CC2)co1</smiles>

66: Diglucotol

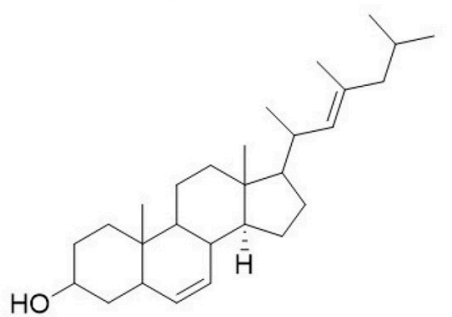

65: Ergosterol 
<smiles>CCCCCCCCCCCCCCC(O)C(=O)NC(COC1OC(CO)C(O)C(O)C1O)C(O)/C=C/CC/C=C(\C)CCCCCCCCC</smiles>

67: Cerebroside $\mathrm{C}$<smiles>CC(C)C(C)/C=C/[C@@H](C)[C@H]1CC[C@H]2[C@@H]3CC(=O)[C@]4(O)CC(O)CC[C@]4(C)[C@H]3CC[C@@]21C</smiles>

69: 3,5,9-trihydroxy-(22E,24R)ergosta-7,22-dien-6-one<smiles>C[C@H]1CC(O)CC2(C)C3COC(=O)C3=CC[C@H]12</smiles>

72: 2, 14-dihydrox-7drimen-12, 11-olide<smiles>COc1cc(CNC(=O)CCCC/C=C/C(C)C)ccc1O</smiles>

73: Capsaicin

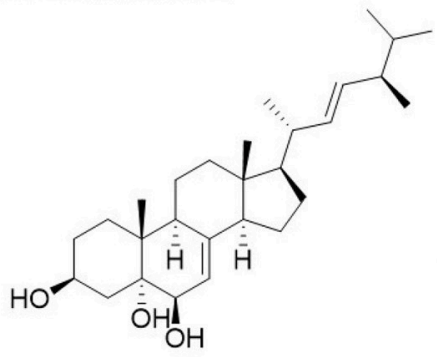

70: Cerevisterol<smiles>CC(=O)NCCc1c[nH]c2ccccc12</smiles>

68: N-acetyltryptamine

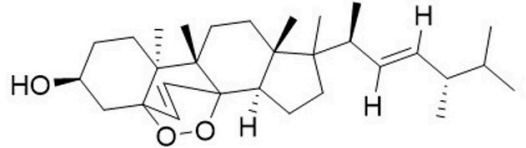

71: Ergosterol peroxide<smiles>CC(C)(C)c1ccc(O)c(C(C)(C)C)c1</smiles>

74: 2,4-di-tert-butyl phenol
$3 \beta, 5 \alpha, 9 \alpha$-trihydroxy-(22E,24R)-ergosta-7,22-dien-6-one (69), cerevisterol (70), and ergosterol peroxide (71), were isolated from Fusarium equiseti from the salt-tolerant plant Salicornia bigelovii Torr. Diglucotol exhibited weak antiproliferative activity toward MCF7, MDA-MB-231, and Caco-2 cells with EC 50 values of $97.56,92.35$, and $99.39 \mu \mathrm{M}$, respectively. Cerevisterol showed potent growth inhibitory activities against MCF7, MDA-MB231, and Caco-2 cancer cells with $\mathrm{EC}_{50}$ values of 32.4, 41.5, and $37.56 \mu \mathrm{M}$, respectively. Ergosterol peroxide exhibited relatively less potency compared with cerevisterol and the $\mathrm{EC}_{50}$ values ranged between 52.4 and $77.56 \mu \mathrm{M}$ (Wang et al., 2014). In another study, 2,14-dihydrox-7-drimen-12,11-olide (72) was isolated from Aspergillus glaucus from the leaves of Ipomoea batatas. Anticancer property of the isolated compound was evaluated in HepG2 and MCF7 cells. The isolated compound displayed moderate cytotoxic effect against HepG2 cells with an IC $_{50}$ value of $61 \mu \mathrm{g} / \mathrm{mL}$ and possessed good cytotoxicity against MCF7 cells with $\mathrm{IC}_{50}$ values of $41.7 \mu \mathrm{g} / \mathrm{mL}$ (Mohamed et al., 2013).

Capsaicin (73) along with 2,4-di-tert-butyl phenol (74) and a novel compound alternariol-10-methyl ether (75) was isolated from Alternaria alternata of Capsicum annuum. Alternariol10-methyl ether displayed considerable antiproliferative activity against a panel of human cancer cell lines (HL-60, A431, A549, PC-3, HeLa, MIA PaCa-2, and T47D). Formation of apoptotic bodies, loss of mitochondrial membrane potential and fragmentation of genomic DNA are some of the morphological and electrochemical changes that occur in the cell undergoing to apoptosis (Rakesh et al., 2014; Roopashree et al., 2015). On treatment of HL-60 with alternariol-10-methyl ether, change in the nuclear morphology and loss of mitochondrial membrane potential was observed (Devari et al., 2014).

In another study, endophytes were isolated from different parts of hazelnut (Corylus avellana L.) and the isolate was identified as Phomopsis amygdali. Further analysis revealed the presence of two metabolites namely (S)-4-butoxy-6((S)-1-hydroxypentyl)-5,6-dihydro-2H-pyran-2-one (76) and pestalotin (77). Both compounds were tested for cytotoxicity against MDA-MB-231, PC-3, HT-29, and HEK293 cell lines. Compound 76 presented good activity against MDA-MB-231 $\left(\mathrm{IC}_{50}: 24.26 \mu \mathrm{g} / \mathrm{mL}\right.$ ) and moderate activity against PC-3 and HT-29 (132.23 and $82.18 \mu \mathrm{g} / \mathrm{mL}$, respectively). On the other hand, pestalotin showed good cytotoxic activity against the PC-3, HT-29, and MDA-MB-231 cell lines with $\mathrm{IC}_{50}$ of 20.54, 16.69 , and $41.70 \mu \mathrm{g} / \mathrm{mL}$ respectively (Akay et al., 2014). In the same study, authors investigated the presence of gene region of taxadiene synthase (a key enzyme in taxol biosynthesis) in the Phomopsis amygdali isolate using PCR. The results demonstrated the absence of Ts gene region indicating that isolate may not produce taxol. 
<smiles>COc1ccc(O)c2c(=O)oc3cc(O)cc(C)c3c12</smiles>

75: Alternariol-10-methyl ether<smiles>CCCCOC1=CC(=O)O[C@H]([C@@H](O)CCCC)C1</smiles>

76: (S)-4-butoxy-6-((S)-1-hydroxypentyl) -5,6-dihydro-2H-pyran-2-one<smiles>CCCC[C@H](O)[C@H]1CC(OC)=CC(=O)O1</smiles>

77: Pestalotin
The Aspergillus sp. fungus was isolated from medicinal plant Gloriosa superba. The bioassay-guided fractionation resulted in an isolation of a novel compound colchatetralene (78) along with the three known compounds namely 5-(hydroxymethyl)furan-2 carbaldehyde (79), 4-hydroxy-phthalic acid-dimethyl ester (80), and ergosterol (65). All four compounds were subjected to their cytotoxic effect against A549, HEp2, MCF7, OVCAR5, THP1, and CV1 cell lines. Colchatetralene was found to be effective against THP-1 and MCF7 with an $\mathrm{IC}_{50}$ value of 30 and $50 \mu \mathrm{g} / \mathrm{mL}$, respectively (Budhiraja et al., 2013). In another report, two strains of the endophytic fungi Penicillium melinii Yuan-25 and Penicillium janthinellum Yuan-27 were isolated from the roots of Panax ginseng at Changchun, Jilin Province, China. Bioactivity oriented isolation from the Yuan-25 culture yielded a new benzaldehyde derivative, ginsenocin (81), along with five known compounds, methyl 2,4-dihydroxy 3,5,6trimethylbenzoate (82), 3,4,5-trimethyl-1,2-benzenediol (83), penicillic acid (84), mannitol (85), and ergosterol peroxide (71). On the other hand, brefeldin A (11) was isolated as a major constituent from the Yuan-27 culture. The isolated compounds were tested for their cytotoxic potential against six human cancer cell lines (MKN45, LOVO, A549, MDA-MB-435, HepG2, and HL-60). Brefeldin A exhibited the maximum cytotoxicity against the all tested cell lines with $\mathrm{IC}_{50}$ values less than $0.12 \mu \mathrm{g} / \mathrm{mL}$.
Ginsenocin and penicillic acid also showed effective cytotoxicity with $\mathrm{IC}_{50}$ values ranging from 0.49 to $7.46 \mu \mathrm{g} / \mathrm{mL}$ against tested cancer cells (Zheng et al., 2013).

The endophytic fungus $A$. tenuissima was obtained from the bark of Erythrophleum fordii Oliver at Nanning, Guangxi Province, China. Tenuissimasatin (86), a novel isocoumarin, and other 11 known metabolites were isolated from A. tenuissima. Coumarin derivatives are known for their anticancer properties and their effect on various cancer models is well-documented (Keerthy et al., 2014; Neelgundmath et al., 2015). Among the 12 compounds (6aR,6bS,7S)-3,6a,7,10-tetra-hydroxy-4,9-dioxo4,6a,6b,7,8,9 hexahydroperylene (87) illustrated good cytotoxic activity toward HCT-8 cells with $\mathrm{IC}_{50}$ value of $1.78 \mu \mathrm{mol} / \mathrm{L}$. Other compounds showed relatively less activity with $\mathrm{IC}_{50}$ value more than $10 \mu \mathrm{M} / \mathrm{L}$ (Fang et al., 2012). A novel metabolite, chaetoglobosin $\mathrm{X}(\mathbf{8 8})$, together with three known compounds (erogosterol, ergosterol 5,8-peroside, and 2-methyl-3-hydroxy indole) were isolated from the endophyte Chaetomium globosum Kunze. The fungus was isolated from the medicinal plant Curcuma wenyujin collected from Zhejiang Province, Wenzhou, China. Chaetoglobosin X exhibited strong cytotoxic activity against $\mathrm{H} 22$ cells with an $\mathrm{IC}_{50}$ value of $3.125 \mu \mathrm{g} / \mathrm{mL}$ and moderate cytotoxicity against $\mathrm{MFC}$ with an $\mathrm{IC}_{50}$ value of $6.25 \mu \mathrm{g} / \mathrm{mL}$ (Wang et al., 2012).<smiles>CC(=O)NC1Cc2cc(O)c(O)c(O)c2C2C=CC(=O)C=CC12</smiles>

78: Colchatetralene<smiles>COC(=O)c1c(C)c(C)c(O)c(C)c1O</smiles>

82: Methyl 2,4-dihydroxy-3,5,6trimethylbenzoate
$\mathrm{HO}$<smiles>Cc1ccc(C=O)o1</smiles>

79: 5-(hydroxymethyl)furan-2carbaldehyde<smiles>Cc1cc(O)c(O)c(C)c1C</smiles>

83: 3,4,5-trimethyl1,2-benzenediol<smiles>COC(=O)c1ccc(O)cc1C(=O)OC</smiles>

80: 4-hydroxy-phthalic acid-dimethyl ester<smiles>C=C(C)C1(O)OC(=O)C=C1OC</smiles>

84: Penicillic acid<smiles>Cc1c(O)cc(/C=C2\C=CC[C@H](C)O2)c(C=O)c1O</smiles>

81: Ginsenocin<smiles>OC[C@@H](O)[C@@H](O)[C@H](O)[C@H](O)CO</smiles>

85: Mannitol 
<smiles>COC(=O)C1(C)Cc2cccc(O)c2C(=O)O1</smiles>

86: Tenuissimasatin<smiles>O=C1C[C@H](O)[C@H]2c3c(ccc(O)c31)-c1ccc(O)c3c1[C@@]2(O)C=CC3=O</smiles>

87: (6aR,6bS,7S)-3,6a,7,10-tetra -hydroxy-4,9 dioxo-4,6a,6b, 7,8,9 hexahydroperylene<smiles>CC1=CC(C)(C(C(=O)O)C(=O)C(C)CC(C)=C(C)CCc2ccc(C)o2)OC1=C1C(O)=C(O)C(O)=C1O</smiles>

88: Chaetoglobosin $\mathrm{X}$
The bioactive metabolites of Penicillium sp., isolated from the leaves of Hopea hainanensis are monomethylsulochrin (89), rhizoctonic acid (90), asperfumoid (91), physcion, 7,8dimethyl-isoalloxazine, and 3,5-dichloro-p-anisic acid (92). All the six compounds were evaluated for cytotoxicity against $\mathrm{KB}$ and HepG2 cells. The compounds 89, 90, 91, and 92 exhibited good cytotoxicity with $\mathrm{IC}_{50}$ values of 30,20 , 20 , and $5 \mu \mathrm{g} / \mathrm{mL}$ for $\mathrm{KB}$ cells; 30, 25, 15, and $10 \mu \mathrm{g} / \mathrm{mL}$ for HepG2 cells respectively (Wang et al., 2008). A novel chlorinated azaphilone derivative named chaetomugilin D (93), together with three known metabolites, chaetomugilin A (94), chaetoglobosin A (95) and C (96), were acquired from C. globosum. The endophytic fungus was isolated from the leaves of G. biloba in Linyi, Shandong province, China. All the isolated compounds displayed significant growth inhibitory activity against brine shrimp (Artemia salina) and Mucor miehei (Qin et al., 2009).

The novel alkaloids chaetoglobosin V (97) and W (98), together with six known congeners were obtained from C. globosum isolated from Artemisia annua at Nanjing, Jiangsu Province, China. Chaetoglobosin V and $\mathrm{W}$ displayed moderate cytotoxic activity against MCF7 and HepG2 cell lines with $\mathrm{IC}_{50}$ values of 52.76 and $51.04 \mu \mathrm{M}$, respectively. The cytotoxicity of chaetoglobosin $\mathrm{V}$ and $\mathrm{W}$ was also assessed against KB, K562, MCF7, and HepG2 cells. Chaetoglobosin V displayed the $\mathrm{IC}_{50}$ value of 23.53 and $27.86 \mu \mathrm{g} / \mathrm{mL}$ in $\mathrm{KB}$ and MCF7 cells whereas chaetoglobosin $\mathrm{W}$ showed 21.17 and $27.87 \mu \mathrm{g} / \mathrm{mL}$ against the same cells, respectively. However, both the compounds did not show cytotoxicity in K562 cells up to $30 \mu \mathrm{g} / \mathrm{mL}$ (Zhang J. et al., 2010).<smiles>COC(=O)c1cc(O)cc(OC)c1C(=O)c1c(O)cc(C)cc1OC</smiles>

89: Monomethylsulochrin 90: Rhizoctonic acid<smiles>COC1=CC(=O)C(O)=C(OC)C12C(=O)Nc1cc(C)cc(OC)c1C2=O</smiles>

91: Asperfumoid<smiles>COc1c(Cl)cc(C(=O)O)cc1Cl</smiles>

92: 3,5-dichloro-p-anisic acid<smiles>CCC(C)/C=C/C1=CC2=C(Cl)C(=O)[C@H]3O[C@@]4(O)[C@@H](C)C(C)OC(=O)[C@H]4[C@H]3C2=CO1</smiles>

93: Chaetomugilin D<smiles>CC(O)C(C)/C=C/C1=CC2=C(Cl)C(=O)[C@@]3(C)O[C@@]4(O)[C@@H](C(=O)O[C@H](C)[C@@H]4C)[C@H]3C2=CO1</smiles>

94: Chaetomugilin A

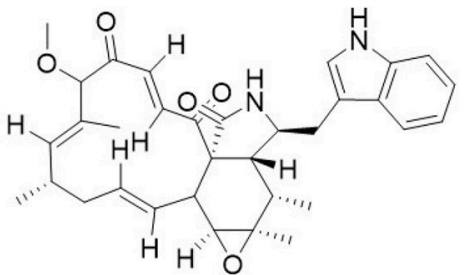

95: Chaetoglobosin A

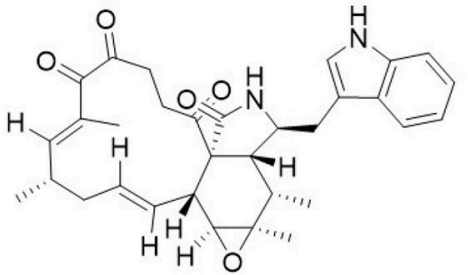

96: Chaetoglobosin C

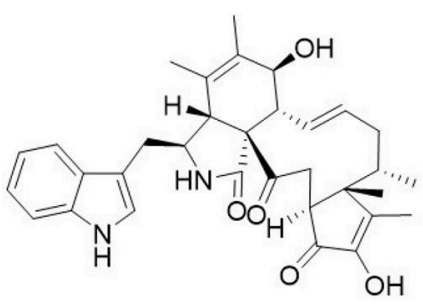

97: Chaetoglobosin V

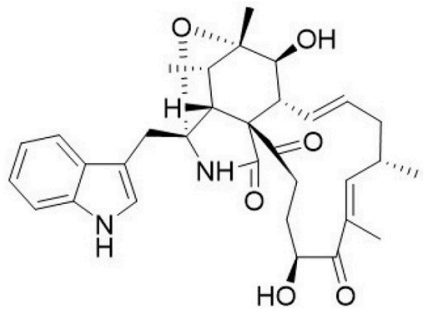

98: Chaetoglobosin W 
An endophytic fungus, Botryotinia fuckeliana isolated from Ajuga decumbens Thunb, produced two new (1-keto-4a,15epoxyeudesm-11-ol and ent-4(15)-eudesmen-5,6-ol-1-one) and four known (ent-4(15)-eudesmen-11-ol-1-one, phenochalasin B, [12]-cytochalasin, [1,3] dioxacyclotridecino) metabolites. Among the isolated compounds, phenochalasin B (99) and [12]cytochalasin (100) exhibited significant inhibitory activity with $\mathrm{IC}_{50}$ values of 7.43 and $0.88 \mu \mathrm{M}$ for SMMC-7721 cells; 0.83 and $0.66 \mu \mathrm{M}$ for A549 cells; 2.22 and $0.062 \mu \mathrm{M}$ for HepG2 cells; and 0.10 and $0.59 \mu \mathrm{M}$ for MCF7 cells respectively. The cytotoxic effect of phenochalasin B was significant on A549, HepG2, and MCF7 cancer cells compared with the SMMC-7721 cells (Lin et al., 2015).

Rohitukine (101) is a chromane alkaloid with anticancer, anti-inflammatory, and immunomodulatory properties. The endophytic fungus, Fusarium proliferatum was isolated from the inner bark of the tree Dysoxylum binectariferum Hook.f growing in Central Western Ghats of Karnataka, India. The yield of rohitukine was $186 \mu \mathrm{g} / 100 \mathrm{~g}$ dry mycelial weight. The methanolic extract of $F$. proliferatum was found to be cytotoxic against the HCT-116 and MCF7 cell lines with an $\mathrm{IC}_{50}$ value of $10 \mu \mathrm{g} / \mathrm{mL}$ for both the cell lines and the $\mathrm{IC}_{50}$ value of positive control (Camptothecin) was found to be $1 \mu \mathrm{g} / \mathrm{mL}$ (Mohana Kumara et al., 2012). The culture of F. oxysporum was isolated from the bark of Cinnamomum kanehirae from Jiaoban mountain, Taiwan province. The fungal metabolite analysis revealed the presence of two new compounds namely oxysporidinone analog (102), 3-hydroxyl-2-piperidinone derivative (103), along with seven known compounds namely (-)-4,6'-anhydrooxysporidinone (104), (+)-fusarinolic acid (105), gibepyrone D (106), beauvercin (107), cerevisterol (70), fusaruside (108), and (2S,2'R,3R, 3'E,4E,8E)-1-O-D-glucopyranosyl-2-N-(2' -hydroxy$3^{\prime}$-octadecenoyl)-3-hydroxy-9-methyl-4,8-sphingadienine

(109). Subsequently, all the compounds were evaluated for cytotoxicity against the PC-3, Panc-1, and A549. The results showed that beauvericin is moderately cytotoxic against PC-3, Panc-1, and A549 with $\mathrm{IC}_{50}$ values of 49.5, 47.2, and
10.4 $\mu \mathrm{M}$. The other compounds displayed weak cytotoxicity for all the tested cell lines (Wang et al., 2011). Endophytes from Clidemia hirta (a perennial shrub) were isolated, and analyses of the endophytic Cryptosporiopsis sp., led to the isolation of three bioactive molecules, (R)-5-hydroxy-2methylchroman-4-one (110), and two novel compounds 1-(2,6-dihydroxyphenyl)pentan-1-one (111), and (Z)-1-(2(2-butyryl-3-hydroxyphenoxy)-6-hydroxyphenyl)-3-hydroxy but-2-en-1-one (112). Compound 110 was presented as a lead cytotoxic agent against the HL-60 with $\mathrm{IC}_{50}$ of $4 \mu \mathrm{g} / \mathrm{mL}$. This compound significantly induced arrest of cell cycle in G2 phase in HL-60 cells (Zilla et al., 2013). Inflammation is called as double-edged sword because deregulation in inflammatory signaling pathways may lead to cancer (Srinivas et al., 2015). Therefore, inflammation and cancer are closely entangled. In the next study, the endophytic fungus Cs-c2 (Phomopsis sp.) was isolated from healthy adult leaves of Senna spectabilis (Fabaceae). Thereafter, secondary metabolites were extracted and evaluated the anti-inflammatory activities. The chemical investigation of the fungus revealed the presence of a new compound (2-hydroxy-alternariol) together with six known compounds (cytochalasins $\mathrm{J}$ and $\mathrm{H}, 5$-epialtenuene, alternariol monomethyl ether, alternariol, and cytosporone C). Cytochalasin $\mathrm{J}$ and $\mathrm{H}$, and alternariol exhibited good antiinflammatory activity (Chapla et al., 2014). In another study, hydroxy-2-methoxy-5-methylpyridin-2(1H)-one ( $\alpha$-pyridone derivative), 3-hydroxy-N-(1-hydroxy-3-methylpentan-2-yl)-5oxohexanamide (a ceramide derivative), and 3-hydroxy-N-(1hydroxy-4-methylpentan-2-yl)-5-oxohexanamide (a new natural compound) along with 15 known compounds were isolated from an endophytic fungus Botryosphaeria dothidea KJ-1. The fungus was collected from the symptomless tissue of stem bark of Melia azedarach L. (white cedar) at Yangling, Shaanxi province, China. The results presented that the new compounds did not induce a significant cytotoxic effect on HCT116 cells up to $100 \mu \mathrm{M}$. However, the known compounds exhibited cytotoxic effect with $\mathrm{IC}_{50}$ values ranging between 3.13 and $73.4 \mu \mathrm{M}$ (Xiao et al., 2014).

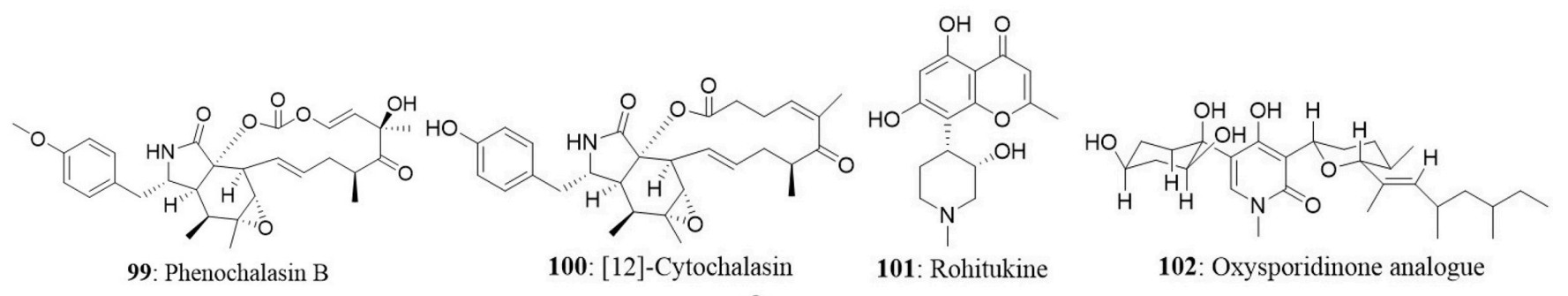

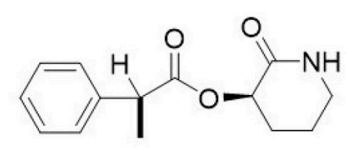

103: 3-hydroxyl-2-piperidinone derivative

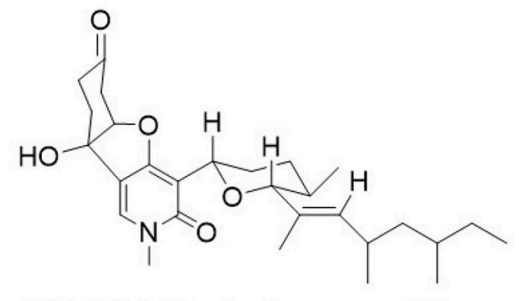

104: (-)-4,6'-anhydrooxysporidinone

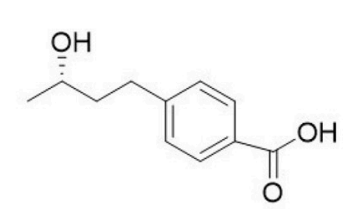

105: (+)-Fusarinolic acid 
<smiles>C/C(=C/C(=O)O)c1ccc(C)c(=O)o1</smiles>

106: Gibepyrone D

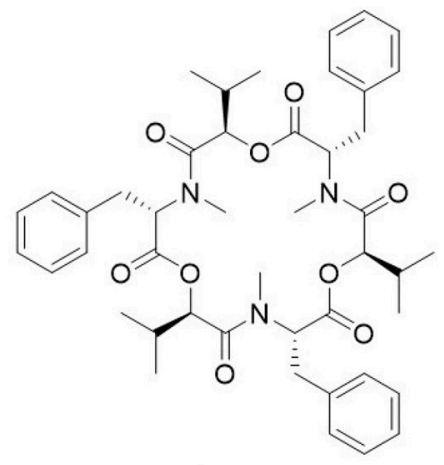

107: Beauvercin<smiles>C[C@H]1CC(=O)c2c(O)cccc2O1</smiles>

110: (R)-5-hydroxy-2methylchroman-4-one

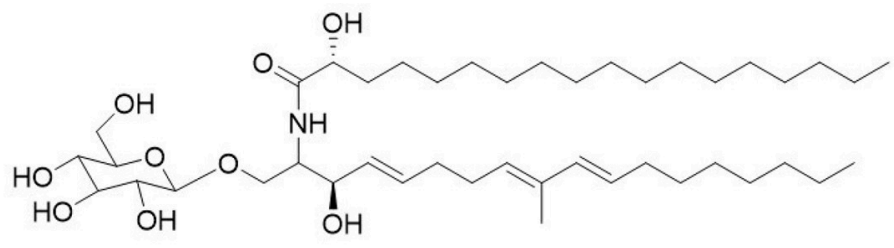

108: Fusaruside<smiles>CCCCCCCCCCC/C=C/[C@@H](O)C(=O)NC(COC1OC(O)C2(O)COC1C(O)C(O)C2O)[C@@H](O)/C=C/C=C/CC/C=C(\C)CCCCCCCCCC</smiles>

109: (2S,2R,3R,3E,4E,8E)-1-O-D-glucopyranosyl-2-N-(2-hydroxy3-octadecenoyl)-3-hydroxy-9-methyl-4,8-sphingadienine<smiles>CCCCC(=O)c1c(O)cccc1O</smiles>

111: 1-(2,6-dihydroxyphenyl) pentan-1-one<smiles>CCCC(=O)c1c(O)cccc1Oc1cccc(O)c1C(=O)C=C(C)O</smiles>

112: (Z)-1-(2-(2-butyryl-3-hydroxy phenoxy)-6-hydroxyphenyl)-3hydroxybut-2-en-1-one

\section{CYTOTOXIC METABOLITES OF ENDOPHYTIC FUNGI FROM MANGROVE PLANTS}

Mangrove endophytic fungi are the second largest ecological group of marine fungi and are potential sources for isolation of new bioactive compounds (Kalaiselvam, 2015). They have attracted researchers due to their importance in ecology (Calcul et al., 2013). Presently, only a small number of mangrove fungi have been studied. Fungal endophytic associations with mangrove allow them to successfully compete with saprobiotic fungi for decomposition of their senescent parts (Elavarasi et al., 2012). Mangrove-associated fungi provide a wide array of bioactive secondary metabolites with unique structures including alkaloids, benzopyranones, chinones, flavonoids, phenolic acids, quinones, steroids, terpenoids, tetralones, and xanthones (Huang et al., 2011). In the following section, natural compounds isolated from endophytic fungi from mangrove plants have been discussed.

Seven fungal strains were isolated from the leaves of Rhizophora mucronata and Avicennia officinalis. One of the isolated endophytic fungi obtained was identified as Irpex hynoides. The fungal extract showed potent cytotoxic activity against HEp2 cell line and the bioactive metabolite in the extract was predicted to be tetradecane (Bhimba et al., 2011). The endophytic fungus Zh6-B1 from Sonneratia apetala produces two new metabolites namely 3R,5R-Sonnerlactone (113), 3R,5S-Sonnerlactone (114), and two known compounds namely 3,4-dihydro-4,8-dihydroxy-7-(2-hydroxyethyl)-6methoxy-1(2H)-naphthalen-1-one and 10-norparvulenone. The antiproliferative activity of new compounds was evaluated against multi-drug resistant human oral floor carcinoma (KV/MDR) cells. Both the compounds (113 and 114) inhibited $\mathrm{KV} / \mathrm{MDR}$ cell growth by 42.4 and $41.6 \%$ at $100 \mu \mathrm{M}$, respectively (Li et al., 2010). The endophyte F.oxysporum from Rhizophora annamalayan was identified as taxol-producing fungus. The amount of taxol produced by the fungus was found to be $172.3 \mu \mathrm{g} / \mathrm{L}$ (Elavarasi et al., 2012). Anthracenedione derivatives were extracted from the secondary metabolites of mangrove endophytic fungi Halorosellinia sp. (No. 1403) and Guignardia sp. (No. 4382) isolated from Kandelia woody tissue from the South China Sea. Of the 14 compounds investigated, 1-hydroxy-3-methylanthracene-9,10-dione (115) induced apoptosis in $\mathrm{KB}$ and $\mathrm{KBv} 200$ cells and displayed cytotoxicity with $\mathrm{IC}_{50}$ values of 3.17 and $3.21 \mu \mathrm{M}$ respectively (Zhang J. Y. et al., 2010). The endophyte Phomopsis sp. ZSU-H76 produced a new polyketide, 2-(7'-hydroxyoxooctyl)-3-hydroxy5-methoxybenzeneacetic acid ethyl ester (116) and three known 
compounds (Dothiorelone A, B, and C). This endophyte was isolated from the stem of Excoecaria agallocha in Dong Zai, Hainan, China. The polyketide exhibited cytotoxicity toward HEp2 and HepG2 cell lines with $\mathrm{IC}_{50}$ values of 25 and $30 \mu \mathrm{g} / \mathrm{mL}$, respectively (Huang et al., 2009). The endophytic fungus Paecilomyces sp. was isolated from the bark of an unidentified mangrove from the Taiwan Strait and it was found to produce two new depsidone-type metabolites named paeciloxocin A (117) and B (118). Paeciloxocin A exhibited significant cytotoxicity against HepG2 cell line with the $\mathrm{IC}_{50}$ value of $1 \mu \mathrm{g} / \mathrm{mL}$, whereas paeciloxocin B showed less cytotoxic activity with an $\mathrm{IC}_{50}$ value of $65 \mu \mathrm{g} / \mathrm{mL}$ (Wen et al., 2010).

The culture of Penicillium expansum was isolated from roots of the mangrove plant Excoecaria agallocha (Euphorbiaceae). The fungus yielded four new polyphenols [expansol A (119), B (120), (S)-(+)-11-dehydrosydonic acid (121), and (7S,11S)-(+)-12acetoxysydonic acid (122)] and two known compounds $[(S)-(+)-$ sydonic acid and diorcinol]. Expansol A and B contain phenolic bisabolane sesquiterpenoid as well as diphenyl ether units in their structure. All the isolated compounds were evaluated for cytotoxicity against the A549 and HL-60 cell lines. Expansol A presented moderate antiproliferative activity against the HL-60 cell line with the $\mathrm{IC}_{50}$ value of $15.7 \mu \mathrm{M}$, whereas expansol $\mathrm{B}$ remarkably suppressed the proliferation of both cell lines with $\mathrm{IC}_{50}$ values of 1.9 and $5.4 \mu \mathrm{M}$, respectively (Lu et al., 2010).

Mangrove-derived endophytic fungus Fusarium sp. was isolated from the fresh stems of Kandelia candel
(Rhizophoraceae) and metabolite analysis revealed the presence of a new isoflavone [5-O-methyl-2'-methoxy$3^{\prime}$-methylalpinumisoflavone (123)] together with four known compounds [6-methoxy-5,7,4'-trihydroxyisoflavone, 6-methoxy-7,4'-dihydroxyisoflavone, (+)-marmesin and 4methylbenzoic acid carboxymethyl ester]. Cytotoxic activity studies against the HEp2 and HepG2 cell lines revealed that compound 123 inhibit cell proliferation significantly with $\mathrm{IC}_{50}$ values of 4 and $11 \mu \mathrm{M}$, respectively (Huang et al., 2010).

Three new bianthraquinone derivatives, alterporriol K (124), $\mathrm{L}$ (125), and $\mathrm{M}$ (126), together with six known compounds (physcion, marcrospin, dactylariol, tetrahydroaltersolanol B, alternariol, and alternariol methyl ether) were isolated from Alternaria sp. ZJ9-6B from Aegiceras corniculatum collected from the South China Sea. In preliminary studies, Alterporriol $\mathrm{K}$ and $\mathrm{L}$ imparted moderate growth inhibitory activity against MDA-MB-435 and MCF7 cells with $\mathrm{IC}_{50}$ values ranging from 13.1 to $29.1 \mu \mathrm{M}$ (Huang et al., 2011). An ergochrome class mycotoxin, secalonic acid D (127) was isolated from the endophytic fungus No. ZSU44 and evaluated for its anticancer activity against HL60 and K562 cells. The results showed strong cytotoxicity towards HL60 and K562 cells with $\mathrm{IC}_{50}$ values of 0.38 and $0.43 \mu \mathrm{M}$, respectively. Furthermore, apoptosis inducing effect of secalonic acid D was confirmed by annexin V-FITC/PI staining. Activation of executioner caspase and PARP cleavage are the crucial events in the cells undergoing apoptosis (Ashwini et al., 2015; Baburajeev et al., 2015).<smiles>COC(C)C[C@@H](O)CCCc1cc(O)cc(O)c1C(=O)O</smiles>

113: 3R, 5R-Sonnerlactone<smiles>C=C(Cc1cc(OC)cc(O)c1C(=C)CCCCCC(C)O)OCC</smiles>

116: 2-(7'-hydroxyoxooctyl)3-hydroxy-5-methoxybenzene acetic acid ethyl ester<smiles>CO[C@H](C)c1ccc(Cc2c(C)cc(Oc3cc(C)cc(O)c3)cc2O)cc1O</smiles>

119: Expansol A<smiles>CC(O)C[C@@H](O)CCCc1cc(O)cc(O)c1C(=O)O</smiles>

114: 3R, 5S-Sonnerlactone<smiles>COc1c(C(O)CC(C)C)ccc2c1Oc1c(O)cc(C)cc1COC2=O</smiles>

117: Paeciloxocin A<smiles>Cc1cc(O)c2c(c1)C(=O)c1ccccc1C2=O</smiles>

115: 1-hydroxy-3-methyl anthracene-9,10-dione<smiles>COc1c(C(CC(C)C)OC(C)=O)ccc2c1Oc1c(O)cc(C)cc1COC2=O</smiles>

118: Paeciloxocin B<smiles>C=C(C)CCC[C@](C)(O)c1ccc(C(=O)O)cc1O</smiles>

121: (S)-(+)-11-dehydrosydonic acid

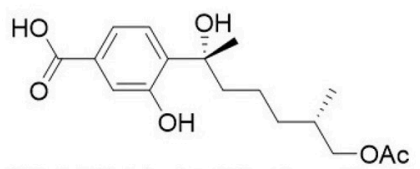

122: (7S,11S)-(+)-11-dehydrosydonic acid 
<smiles>COc1c(-c2coc3cc4c(c(OC)c3c2=O)C=CC(C)(C)C4)ccc(O)c1C</smiles>

123: 5-O-methyl-2'-methoxy-3'methylalpinumisoflavone

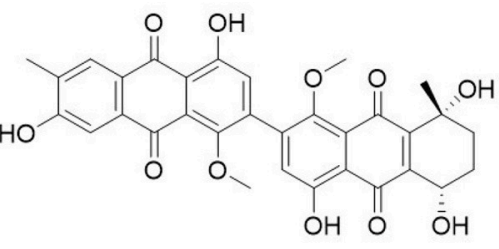

124: Alterporriol $\mathrm{K}$<smiles>COc1c(-c2cc(O)c3c(c2OC)C(=O)c2cc(O)c(O)cc2C3=O)cc(O)c2c1C(=O)C1=C(C[C@H](O)[C@](C)(O)[C@H]1O)C2=O</smiles>

125: Alterporriol L<smiles>COc1c(-c2cc(O)c3c(c2OC)C(=O)c2cc(C)c(O)cc2C3=O)cc(O)c2c1C(=O)C1=C(C[C@@H](O)[C@H](C)[C@H]1O)C2=O</smiles>

126: Alterporriol $\mathrm{M}$<smiles>COC(=O)[C@@H]1OC2=CC=C(C(O)=C1C1=C([OH2+])C[C@@H](C)[C@H](O)[C@H]1C(=O)O)c1ccc3c(c1O)C(=O)C1C(O)CC(C)[C@H](O)[C@@]31OC2=O</smiles>

127: Secalonic Acid D
Secalonic acid D induced the activation of caspase-3 and cleavage of PARP in HL60 and K562 cells (Zhang J. Y. et al., 2009). The endophytic fungus Phomopsis sp. was isolated from Excoecaria agallocha (Euphorbiaceae) in Dong Zai, Hainan, China. The secondary metabolite analysis revealed the presence of three unique compounds namely phomopsisH76 A, B, and C (128-130). These metabolites did not show considerable cytotoxic activity against KB, KBv200, and MCF7 cell lines and $\mathrm{IC}_{50}$ values were over $50 \mu \mathrm{M}$ (Yang et al., 2010).

Three new sesquiterpenes, merulin A (131), B and C (132) were produced by the endophytic fungus XG8D (Basidiomycete) isolated from the mangrove plant Xylocarpus granatum (Meliaceae). Merulin A and C exhibited good cytotoxic activity against human breast and colon cancer cell lines with $\mathrm{IC}_{50}$ values of 4.98 and $1.57 \mu \mathrm{g} / \mathrm{mL}$ for BT474; 4.84 and $4.11 \mu \mathrm{g} / \mathrm{mL}$ for SW620, respectively. Doxorubicin was used as vehicle control with $\mathrm{IC}_{50}$ values of 0.09 and $0.53 \mu \mathrm{g} / \mathrm{mL}$ against SW620 and BT-474 cell lines, respectively. (Chokpaiboon et al., 2010). A new pyrrolyl 4-quinolinone alkaloid, penicinoline (133), was isolated from Penicillium sp., from the bark of the mangrove Acanthus ilicifolius (Acanthaceae) collected from the South China Sea. Intramolecular dehydration of the compound yielded an unexpected lactam derivative (134), which was confirmed by single-crystal X-ray analysis. Penicinoline showed potent cytotoxicity with $\mathrm{IC}_{50}$ values of 0.57 and $6.5 \mu \mathrm{g} / \mathrm{mL}$ toward the 95-D and HepG2 cell lines, respectively. However, penicinoline was inactive against HeLa, KB, KBv200, and HEp2 cell lines at the concentration of $100 \mu \mathrm{g} / \mathrm{mL}$ (Shao et al., 2010).

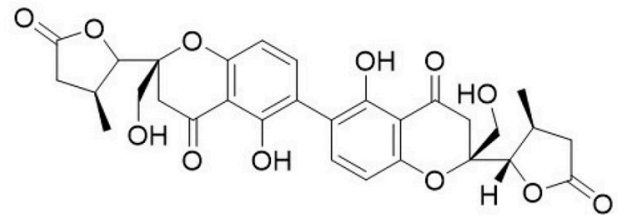

128: Phomopsis-H76 A<smiles>O=c1occ(-c2ccc(O)cc2)c2c1C=CC(=CC=C1C=Cc3c(c(-c4ccc(O)cc4)coc3=O)O1)O2</smiles>

129: Phomopsis-H76 B

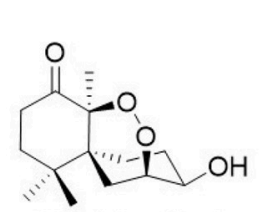

131: Merulin A<smiles>C[C@@]12CCC(CO)(C1)OO[C@@]1(C)CC(O)(C=CC1=O)C2</smiles>

132: Merulin C<smiles>O=C(O)c1c(-c2ccc[nH]2)[nH]c2ccccc2c1=O</smiles>

133: Penicinoline<smiles>O=c1occ(-c2ccc(O)cc2)c2c1C=CC(=CC=C1C=Cc3c(c(-c4ccc(O)cc4[N+](=O)[O-])coc3=O)O1)O2</smiles>

130: Phomopsis-H76 C<smiles>Cn1c2c(c(=O)c3ccccc31)C(=O)n1cccc1-2</smiles>

134: Lactam derivative
The endophytic Pestalotiopsis sp. was obtained from the leaves of Rhizophora mucronata from Dong Zhai Gang, Mangrove Garden on Hainan Island, China. The endophyte produced five novel cytosporones J-N (135-139), five new pestalasins AE (140-144), and a new alkaloid pestalotiopsoid A (145). All the isolated compounds were investigated for their cytotoxicity against L5178Y, HeLa, and PC12 cells. All the compounds were found to be inactive at a concentration of $10 \mu \mathrm{g} / \mathrm{mL}$ (Xu et al., 2009b). The Pestalotiopsis sp. produced six new pestalotiopsones A-F (chromones) (146-151) and a new compound 7-hydroxy-2(2-hydroxypropyl)-5-methylchromone. The fungus was isolated from the leaves of Rhizophora mucronata. Among the isolates, 
<smiles>CC(O)CCCCC1OC(=O)Cc2cc(O)cc(O)c21</smiles>

135: Cytosporone J<smiles>[Y]#CC(C)(C)C(C)C</smiles><smiles>CC(=O)OCCCCCCCC1OC(=O)Cc2cc(O)cc(O)c21</smiles>

137: Cytosporone L<smiles>COC(=O)Cc1cc(O)cc(O)c1C(=O)CCCCCC(C)O</smiles>

138: Cytosporone $\mathrm{M}$<smiles>CCCCCCCC(=O)c1c(O)cc(O)cc1CC(=O)OC</smiles>

139: Cytosporone $\mathrm{N}$<smiles>COc1cc(OC)c2oc(=O)c(CC(C)C)cc2c1</smiles>

140: Pestalasin A

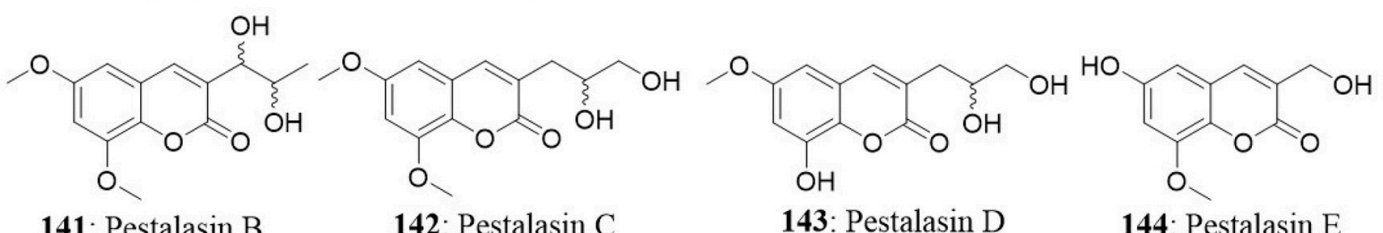

141: Pestalasin $B$

142: Pestalasin C

143: Pestalasin D

144: Pestalasin E<smiles>COC(=O)CCN1C(=O)C(=C(C)C)C(O)C1=O</smiles>

145: Pestalotiopsoid A<smiles>CCCCCCCc1cc(=O)c2c(CC(=O)OC)cc(O)cc2o1</smiles>

146: Pestalotiopsone A<smiles>CCCCCCCc1cc(=O)c2c(CC(=O)OCC)cc(O)cc2o1</smiles>

147: Pestalotiopsone B<smiles>CCCCCCCc1cc(=O)c2c(CC(=O)O)cc(O)cc2o1</smiles>

148: Pestalotiopsone C<smiles>COC(=O)Cc1cc(O)cc2oc(CCCCC[C@H](C)O)cc(=O)c12</smiles>

149: Pestalotiopsone D<smiles></smiles>

150: Pestalotiopsone E

151: Pestalotiopsone F only pestalotiopsone $\mathrm{F}$ exhibited moderate cytotoxicity against the murine cancer cell line (L5178Y) with an $\mathrm{EC}_{50}$ value of $8.93 \mu \mathrm{g} / \mathrm{mL}$ (Xu et al., 2009a).

Five highly oxygenated chromones named rhytidchromones A-E (152-156) were isolated from the endophytic fungus Rhytidhysteron rufulum BG2-Y. The fungal strain was isolated from the healthy leaves of Bruguiera gymnorrhiza collected from Pak Nam Pran, Prachuab Kiri Khan Province, Thailand.
Isolated compounds were tested for their cytotoxicity on a panel of cancer cell lines (MCF7, HepG2, Kato-3, and CaSki). All the compounds excluding rhytidchromone D, displayed cytotoxicity toward Kato-3 cell lines with $\mathrm{IC}_{50}$ values ranging from 16.0 to $23.3 \mu \mathrm{M}$, while rhytidchromones $\mathrm{A}$ and $\mathrm{C}$ were active against MCF7 cells with $\mathrm{IC}_{50}$ values of 19.3 and $17.7 \mu \mathrm{M}$, respectively (Chokpaiboon et al., 2016).<smiles>COc1cc(O)c2c(=O)cc(C)oc2c1C1CC(=O)OC1=O</smiles>

152: Rhytidchromone A<smiles>COC(=O)C(Cc1c(OC)cc(O)c2c(=O)cc(C)oc12)OC</smiles>

153: Rhytidchromone B<smiles>COC(=O)C(CC(OC)c1c(OC)cc(O)c2c(=O)cc(C)oc12)OC</smiles>

154: Rhytidchromone C 155: Rhytidchromone $D$<smiles>COc1cc(O)c2c(=O)cc(C)oc2c1C(CC(OC)C(=O)O)OC</smiles>

156: Rhytidchromone $\mathrm{E}$ 


\section{ENDOPHYTIC FUNGAL METABOLITES FROM MARINE HABITATS}

Marine fungi comprise of obligate and facultative types; and classically they are defined as a form of ecological, but not a taxonomic group of fungi (Elsebai et al., 2014). The growth and sporulation of obligate marine fungi occur exclusively in marine environment. In case of facultative marine fungi, the growth, and sporulation in the marine habitat are dependent on the physiological adaptations (Raghukumar, 2008). All marine habitats such as marine plants (mangrove plants, algae, seagrasses, and driftwood), marine vertebrates and invertebrates (corals, bivalves, sponges, and crustaceans), and abiotic factors (soil) can host fungal strains. Many reviews have not focused significantly on bioactive natural compounds from endophytic fungi of marine origin. The fungal diversity in marine habitats has been underestimated because of their propensity to form aggregates (Rateb and Ebel, 2011). Sponges and algae contribute majorly as sources of fungi in marine habitats. A thorough investigation of algae and sponges is essential to explore the fungal communities associated with them. The marine environment has extreme conditions like high salinity, more amount of metals in hydrothermal vents, elevated hydrostatic pressure, and low temperatures in the deep sea (Raghukumar, 2008). The organisms that live and thrive in the adverse and extreme conditions can be expected to produce metabolites that are unique and might be of therapeutic interest. Therefore, it is essential to search for exotic and unique metabolite-producing organisms in these unusual environments. Marine-derived fungi are a prolific source of new bioactive compounds with a high degree of structural diversity and equally interesting pharmacological activities, namely, anticancer, antibacterial, antifungal, and antiviral activities, as well as involvement in specific enzyme inhibition activities (Bugni et al., 2004). The bioactive natural compounds from marine fungi have been investigated to smaller extents relatively with their terrestrial counterparts (VitaMarques et al., 2008; Gamal-Eldeen et al., 2009). Several research groups have been involved in the isolation of marine microbes, analysis of their secondary metabolites and evaluation of possible pharmacological properties.

The Talaromyces sp. was isolated from seaweed in Kasai Rinkai Park, Tokyo, Japan. Two novel azaphilones, kasanosins A (157) and B (158) were isolated from cultures of Talaromyces sp. These compounds are evaluated for their ability to inhibit

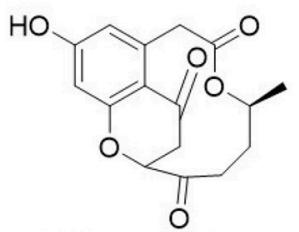

159: Penilactone<smiles>Cc1cc(O)cc(O)c1C(=O)CC1OC1CCCC(C)OC(=O)CO</smiles>

160: 10,11-Epoxycurvularin mammalian DNA polymerases, the enzyme responsible for DNA replication. The results demonstrated that kasanosin $A$ and $B$ selectively inhibit eukaryotic DNA polymerases $\beta$ and $\gamma$. Kasanosin $A$ was more active than kasanosin B, with $\mathrm{IC}_{50}$ values of $27.3 \mu \mathrm{M}$ (rat DNA polymerase $\beta$ ) and $35 \mu \mathrm{M}$ (human DNA polymerase $\lambda$ ), respectively (Kimura et al., 2008). Alterporriol L (52) is a new bianthraquinone derivative isolated from a marine fungus Alternaria sp. ZJ9-6B. The fungus was isolated from the plant Aegiceras corniculatum in the South China Sea. The cytotoxic effect of alterporriol $\mathrm{L}$ was investigated against MCF7 and MDA-MB-435 cells. The $\mathrm{IC}_{50}$ value was found to be $20.04 \mu \mathrm{M}$ for MCF7 and 13.11 $\mu \mathrm{M}$ for MDA-MB-435 (Huang et al., 2012). Penicillium sp. was isolated from Limonium tubiflorum and the chemical investigation of fungal extract revealed the presence of four new compounds namely penilactone (159), 10, 11-epoxycurvularin (160), neobulgarone G (161), and sulfimarin (162) along with 12 known metabolites. NF- $\mathrm{BB}$ is a proinflammatory transcription factor that is persistently activated in several cancers (Neelgundmath et al., 2015; Nirvanappa et al., 2016). The new compounds (159-162) did not show pronounced inhibition against $\mathrm{TNF} \alpha$-induced NF- $\mathrm{B}$ activity in $\mathrm{K} 562$ cells (Aly et al., 2011). The fungus Aspergillus versicolor from the marine sponge Petrosia sp. yielded an aromatic polyketide derivative (2,4-Dihydroxy-6-((R)-4-hydroxy-2-oxopentyl)-3methylbenzaldehyde), two xanthones (sterigmatocystin and dihydrosterigmatocystin) and five anthraquinones (averantin, methylaverantin, averufin, nidurufin, and versiconol). Among the eight isolates, sterigmatocystin (163), averantin (164), methylaverantin (165), and nidurufin (166) exhibited significant cytotoxicity against A549, SK-OV-3, SK-MEL-2, XF-498, HCT15 with $\mathrm{IC}_{50}$ values ranging between 0.41 and $4.61 \mu \mathrm{g} / \mathrm{mL}$ (Lee et al., 2010).<smiles>COc1cc(C)c(C(=O)O[C@H]2CC3=C(COC(/C=C/CO)=C3)C(=O)[C@]2(C)O)c(O)c1</smiles>

157: Kasanosin A<smiles>COc1cc(C)c(C(=O)O[C@]2(C)C(=O)C3=C(C=C(/C=C/CO)OC3)C[C@H]2O)c(O)c1</smiles>

158: Kasanosin B<smiles></smiles>

161: Neobulgarone G<smiles>CS(=O)c1cccc2ccc(=O)oc12</smiles>

162: Sulfimarin 
<smiles></smiles>

163: Sterigmatocystin<smiles>CCCCCC(O)c1cc2c(cc1O)C(=O)c1cc(O)cc(O)c1C2=O</smiles>

164: Averantin<smiles>CCCCCC(OC)c1cc2c(cc1O)C(=O)c1cc(O)cc(O)c1C2=O</smiles>

165: Methylaverantin<smiles>C[C@]12CC[C@@H](O)[C@H](O1)c1c(cc3c(c1O)C(=O)c1c(O)cc(O)cc1C3=O)O2</smiles>

166: Nidurufin

The endophytic fungus Chaetomium globosum (QEN-14) was isolated from the marine green alga Ulva pertusa (Ulvaceae) collected from Qingdao coastline, China. The fungus yielded seven new cytochalasan derivatives (cytoglobosins A$\mathrm{G})$ together with two known compounds (isochaetoglobosin D and chaetoglobosin $\mathrm{F}_{\mathrm{ex}}$ ). New isolates were evaluated for their cytotoxicity against P388, A549, and KB cancer cell lines. The results indicated that cytoglobosins $C(\mathbf{1 6 7})$ and $D(168)$ as potent anticancer agents towards A549 cells with IC $_{50}$ values of 2.26 and $2.55 \mu \mathrm{M}$, respectively (Cui et al., 2010b). The other compounds did not show significant cytotoxic effect up to $10 \mu \mathrm{M}$.

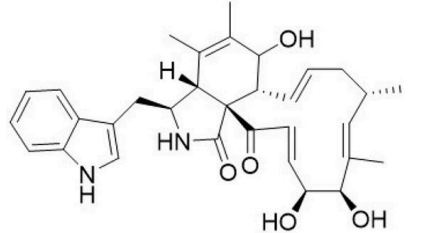

167: Cytoglobosin C

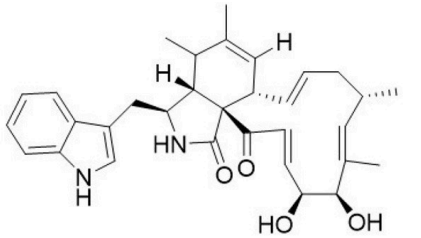

168: Cytoglobosin D
Two prenylated polyketides, epoxyphomalin A (169) and B (170) were obtained from the facultative marine fungus Phoma sp. The fungus Phoma sp. was collected from the marine sponge Ectyplasia perox at the Caribbean Sea, Dominica. The cytotoxic effects of compounds 169 and 170 were evaluated using a monolayer cell survival and proliferation assay on a 36 human tumor cell lines. Epoxyphomalin A displayed considerable in vitro tumor cell selectivity towards 12 of the 36 tested tumor cell lines. The $\mathrm{IC}_{50}$ values were ranged from 0.01 to $0.038 \mu \mathrm{g} / \mathrm{mL}$ (Mohamed et al., 2009). The fungus Curvularia sp. was isolated from the red alga Acanthophora spicifera. Chemical investigations of the fungal isolate yielded eight compounds including apralactone A, antipodes of curvularin macrolides and a dimeric curvularin. A modified propidium iodide monolayer assay was used to determine the cytotoxic activity of the isolated compounds against human tumor cell lines. Apralactone A (171) (14-membered phenylacetic acid macrolactone) showed considerable dose-dependent cytotoxicity with a mean $\mathrm{IC}_{50}$ value of $9.87 \mu \mathrm{M}$. The other compound, (+)(10E,15R)-10,11-dehydrocurvularin (172), also showed dosedependent cytotoxicity with a mean $\mathrm{IC}_{50}$ value of $1.25 \mu \mathrm{M}$ (Greve et al., 2008, 2010).

Chemical investigation of Aspergillus ochraceus isolated from the marine brown alga Sargassum kjellmanianum revealed the production of 7-nor-ergosterolide (173) and two new steroidal derivatives $[3 \beta, 11 \alpha$-dihydroxyergosta-8,24(28)-dien-7-one (174) and $3 \beta$-hydroxyergosta- 8,24(28)-dien-7-one (175)] and nine known related steroids. Compounds 173, 174, and 175 were tested for their cytotoxic activities against NCI-H460, SMMC7721, SW1990, DU145, HepG2, HeLa, and MCF7 cancer cell lines. The 7-nor-ergosterolide showed potent, selective cytotoxic activity against the NCI-H460, SMMC-7721, and SW1990 cells with IC $_{50}$ values of 5,7 and $28 \mu \mathrm{g} / \mathrm{mL}$, respectively. On the other hand, $3 \beta, 11 \alpha$-dihydroxyergosta-8,24(28)-dien-7-one weakly inhibited the growth of the SMMC-7721 cell line with the $\mathrm{IC}_{50}$ value of $28 \mu \mathrm{g} / \mathrm{mL}$ (Cui et al., 2010a).

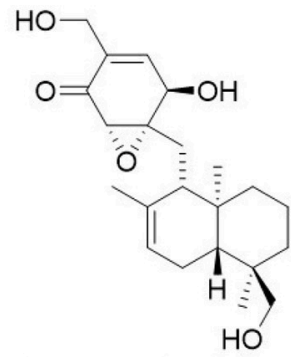

169: Epoxyphomalin A

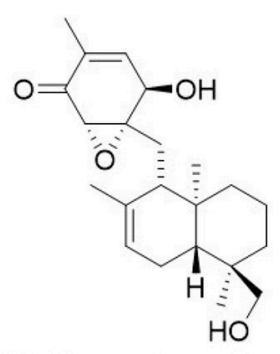

170: Epoxyphomalin B<smiles></smiles>

171: Apralactone A<smiles>CO[C@H](C)CCC/C=C/C(=O)c1c(O)cc(O)cc1CC(=O)O</smiles>

172: (+)-(10E,15R)-10,11 -dehydrocurvularin 


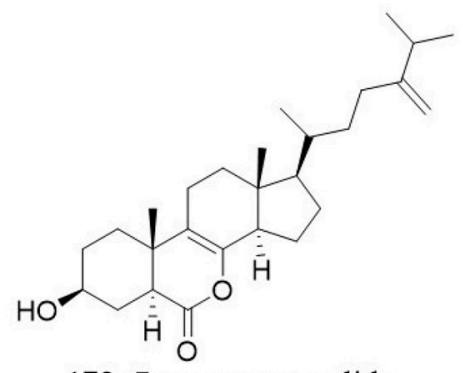

173: 7-nor-ergosterolide

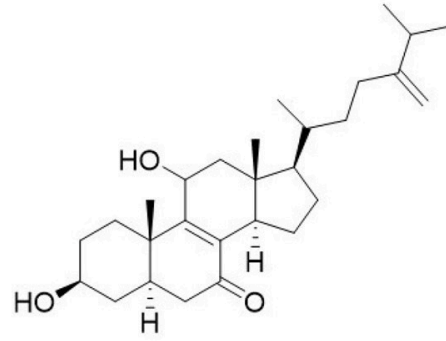

174: 3,11-dihydroxyergosta -8,24(28)-dien-7-one

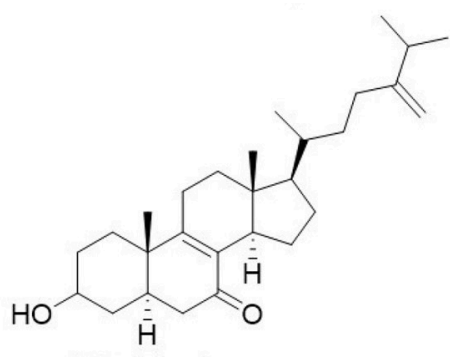

175: 3-hydroxyergosta $-8,24(28)$-dien-7-one

\section{FUTURE CHALLENGES WITH ENDOPHYTES}

Endophytic fungi offer new hope and possibilities for the production of novel bioactive compounds, which is challenged due to low yield, lack of information on precise fungiplant biochemical interaction, scale-up issues, and growth in the axenic state. There is a constant need to manifest these natural secondary metabolites in vitro, which despite being accomplished, has shown limited success in commercial production. There is a weak understanding of interactions of endophytic fungal organisms with other associated microbes. The use of plant tissue culture techniques to obtain higher yields has not been successful due to genetic instability in culture, slow growth of fungi, the formation of cell aggregates, and sensitivity to shearing (Charlwood and Rhodes, 1990). Additionally, the maintenance of tissue cultures is comparatively more expensive and time-consuming than fermentation technologies. Nevertheless, fungal fermentation provides the platform for the potent and sustainable production of bioactive compounds. Microbial fermentation offers certain advantages such as a simple fungal cell medium, low cost, faster growth rate of fungi with minimal risk of contamination, and in vitro optimized fermentation conditions that facilitate the production of secondary metabolites but fails to meet the requirements for sustained production, as observed in vivo in the host apoplast region. Studies examining genetic aspects to trigger specific genes for overproduction of target metabolites could also be beneficial. Another important hurdle is the difficulties associated with using a combinatorial chemical synthesis approach to generate complex metabolites of the bioactive compounds. These shortcomings may overcome by the use of inducers/elicitors of the specific biochemical pathway that can ultimately increase the yield. The complex structure of endophytic metabolites is a hurdle for use of the combinatorial chemical synthesis approach. Alternatively, the limitations may overcome by the use of signaling molecules and inexpensive precursor feeding in growth medium, which may act as efficient triggers of the biochemical pathway to achieve an elevated yield. Moreover, the presence of several unnecessary metabolites can interfere with the enzymatic activity in the growth medium. The negative feedback mechanism is a major aspect that must be critically investigated to achieve increase in yield with the fungal fermentation strategy (Zhao et al., 2010a). Endophytic studies employing genetic engineering techniques, transformation studies, gene cluster amplification for metabolite overproduction, mutagenesis, medium manipulation, culture condition optimization, and elicitor addition should help to increase the yield of the desired secondary metabolites (Kusari and Spiteller, 2012; Kusari et al., 2012; Venugopalan and Srivastava, 2015).

\section{CONCLUSION}

Endophytes have been emerged as privileged sources of bioactive compounds, since after the discovery of taxol from T. andreanae. The secondary metabolites of endophytes have been used substantially for the sustainable production of therapeutically important compounds. The limited availability of bioactive principles in plant sources could be surpassed by exploiting the chemical entities in the endophytes. With the unfolding knowledge of the anticancer properties of endophytes and current technological advancements in "omics" and fermentation, endophytic fungi may play a lead role in providing a constant supply of chemotherapeutics with high target specificity and minimal side effects at an affordable cost. The attempts in genetic manipulations, overexpressing of the genes that are essential for the production of the specific bioactive metabolite, and other optimization strategies may contribute to the strain improvement and make endophytes as potential drug sources for the future. Therefore, exploring and exploiting of metabolites from endophytes in terrestrial, mangrove, and marine habitats may provide an excellent avenue for the discovery of drug candidates against deadly human diseases.

\section{AUTHOR CONTRIBUTIONS}

All authors listed have made a substantial, direct and intellectual contribution to the work, and approved it for publication.

\section{FUNDING}

The Financial assistance to FU (F1-17.1/2012-13/MANF-201213-MUS-KAR-11899) granted by the Maulana Azad National 
Fellowship (MANF), and University Grants Commission (UGC), New Delhi is gratefully acknowledged. The authors would like to extend their sincere appreciation to the Deanship of Scientific Research at King Saud University for its funding to Research
Group number (RGP-271). VG would like to acknowledge funding from the EU7th Framework Programme for Research, Technological Development and Demonstration Activities under Grant Agreement No. 621364 (TUTIC-Green).

\section{REFERENCES}

Abd-Elsalam, K., and Hashim, A. (2013). Hidden fungi as microbial and nano-factories for anticancer agents. Fungal Genomics Biol. 3:e115. doi: 10.4172/2165-8056.1000e115

Akay, S., Ekiz, G., Kocabaş, F., Hameş-Kocabaş, E. E., Korkmaz, K. S., and Bedir, E. (2014). A new 5,6-dihydro-2-pyrone derivative from Phomopsis amygdali, an endophytic fungus isolated from hazelnut (Corylus avellana). Phytochem. Lett. 7(Suppl. C), 93-96. doi: 10.1016/j.phytol.2013.09.012

Alberts, B. (2017). Molecular Biology of the Cell. New York, NY: Garland science.

Alurappa, R., Bojegowda, M. R., Kumar, V., Mallesh, N. K., and Chowdappa, S. (2014). Characterisation and bioactivity of oosporein produced by endophytic fungus Cochliobolus kusanoi isolated from Nerium oleander L. Nat. Prod. Res. 28, 2217-2220. doi: 10.1080/14786419.2014.924933

Alvin, A., Miller, K. I., and Neilan, B. A. (2014). Exploring the potential of endophytes from medicinal plants as sources of antimycobacterial compounds. Microbiol. Res. 169, 483-495. doi: 10.1016/j.micres.2013.12.009

Aly, A. H., Debbab, A., Clements, C., Edrada-Ebel, R., Orlikova, B., Diederich, M., et al. (2011). NF kappa B inhibitors and antitrypanosomal metabolites from endophytic fungus Penicillium sp. isolated from Limonium tubiflorum. Bioorg. Med. Chem. 19, 414-421. doi: 10.1016/j.bmc.2010.11.012

Aly, A. H., Edrada-Ebel, R., Indriani, I. D., Wray, V., Müller, W. E. G., Totzke, F., et al. (2008). Cytotoxic metabolites from the fungal endophyte alternaria sp. and their subsequent detection in its host plant Polygonum senegalense. J. Nat. Prod. 71, 972-980. doi: 10.1021/np070447m

Amna, T., Puri, S. C., Verma, V., Sharma, J. P., Khajuria, R. K., Musarrat, J., et al. (2006). Bioreactor studies on the endophytic fungus Entrophospora infrequens for the production of an anticancer alkaloid camptothecin. Can. J. Microbiol. 52, 189-196. doi: 10.1139/w05-122

Ashwini, N., Garg, M., Mohan, C. D., Fuchs, J. E., Rangappa, S., Anusha, S., et al. (2015). Synthesis of 1,2-benzisoxazole tethered 1,2,3-triazoles that exhibit anticancer activity in acute myeloid leukemia cell lines by inhibiting histone deacetylases, and inducing p21 and tubulin acetylation. Bioorg. Med. Chem. 23, 6157-6165. doi: 10.1016/j.bmc.2015.07.069

Baburajeev, C. P., Dhananjaya Mohan, C., Ananda, H., Rangappa, S., Fuchs, J. E., Jagadish, S., et al. (2015). Development of novel triazolo-thiadiazoles from heterogeneous "green" catalysis as protein tyrosine phosphatase 1B inhibitors. Sci. Rep. 5:14195. doi: 10.1038/srep14195

Balunas, M. J., and Kinghorn, A. D. (2005). Drug discovery from medicinal plants. Life Sci. 78, 431-441. doi: 10.1016/j.lfs.2005.09.012

Berkowitz, D. B., Choi, S., and Maeng, J. H. (2000). Enzyme-assisted asymmetric total synthesis of (-)-podophyllotoxin and (-)-picropodophyllin. J. Org. Chem. 65, 847-860. doi: 10.1021/jo991582+

Bhimba, B. V., Franco, D. A., Jose, G. M., Mathew, J. M., and Joel, E. L. (2011). Characterization of cytotoxic compound from mangrove derived fungi Irpex hydnoides VB4. Asian Pac. J. Trop. Biomed. 1, 223-226. doi: 10.1016/S2221-1691(11)60031-2

Blagosklonny, M. V. (2004). Analysis of FDA approved anticancer drugs reveals the future of cancer therapy. Cell Cycle 3, 1033-1040. doi: 10.4161/cc.3.8.1023

Blagosklonny, M. V. (2005). Carcinogenesis, cancer therapy and chemoprevention. Cell Death Differ. 12, 592-602. doi: 10.1038/sj.cdd.4401610

Botta, B., Delle Monache, G., Misiti, D., Vitali, A., and Zappia, G. (2001). Aryltetralin lignans: chemistry, pharmacology and biotransformations. Curr. Med. Chem. 8, 1363-1381. doi: 10.2174/0929867013372292

Budhiraja, A., Nepali, K., Sapra, S., Gupta, S., Kumar, S., and Dhar, K. L. (2013). Bioactive metabolites from an endophytic fungus of Aspergillus species isolated from seeds of Gloriosa superba Linn. Med. Chem. Res. 22, 323-329. doi: 10.1007/s00044-012-0032-z

Bugni, T. S., Janso, J. E., Williamson, R. T., Feng, X., Bernan, V. S., Greenstein, M., et al. (2004). Dictyosphaeric acids A and B: new decalactones from an

undescribed Penicillium sp. obtained from the Alga Dictyosphaeria versluyii. J. Nat. Prod. 67, 1396-1399. doi: 10.1021/np049973t

Bunyapaiboonsri, T., Yoiprommarat, S., Srikitikulchai, P., Srichomthong, K., and Lumyong, S. (2010). Oblongolides from the endophytic fungus Phomopsis sp. BCC 9789. J. Nat. Prod. 73, 55-59. doi: 10.1021/np900650c

Calcul, L., Waterman, C., Ma, W. S., Lebar, M. D., Harter, C., Mutka, T., et al. (2013). Screening mangrove endophytic fungi for antimalarial natural products. Mar. Drugs 11, 5036-5050. doi: 10.3390/md11125036

Cao, L., Huang, J., and Li, J. (2007). Fermentation conditions of Sinopodophyllum hexandrum endophytic fungus on production of podophyllotoxin. Food Ferment. Indust. 33:28.

Chakravarthi, B. V., Sujay, R., Kuriakose, G. C., Karande, A. A., and Jayabaskaran, C. (2013). Inhibition of cancer cell proliferation and apoptosis-inducing activity of fungal taxol and its precursor baccatin III purified from endophytic Fusarium solani. Cancer Cell Int. 13:105. doi: 10.1186/1475-2867-13-105

Chakravarthi, B V.., Das, P., Surendranath, K., Karande, A. A., and Jayabaskaran, C. (2008). Production of paclitaxel by Fusarium solani isolated from Taxus celebica. J. Biosci. 33, 259-267. doi: 10.1007/s12038-008-0043-6

Chandra, S. (2012). Endophytic fungi: novel sources of anticancer lead molecules. Appl. Microbiol. Biotechnol. 95, 47-59. doi: 10.1007/s00253-012-4128-7

Chapla, V. M., Zeraik, M. L., Ximenes, V. F., Zanardi, L. M., Lopes, M. N., Cavalheiro, A. J., et al. (2014). Bioactive Secondary Metabolites from Phomopsis sp., an Endophytic Fungus from Senna spectabilis. Molecules 19, 6597-6608. doi: 10.3390/molecules19056597

Charlwood, B. V., and Rhodes, M. J. (1990). Secondary Products from Plant Tissue Culture. New York, NY: Clarendon Press.

Chen, M.-J., Fu, Y.-W., and Zhou, Q.-Y. (2014). Penifupyrone, a new cytotoxic funicone derivative from the endophytic fungus Penicillium sp. HSZ-43. Nat. Prod. Res. 28, 1544-1548. doi: 10.1080/14786419.2014.924932.

Chen, X., Shi, Q., Lin, G., Guo, S., and Yang, J. (2009). Spirobisnaphthalene analogues from the endophytic fungus Preussia sp. J. Nat. Prod. 72, 1712-1715. doi: $10.1021 / \mathrm{np} 900302 \mathrm{w}$

Cheng, M.-J., Wu, M.-D., Yuan, G.-F., Chen, Y.-L., Su, Y.-S., Hsieh, M.T., et al. (2012). Secondary metabolites and cytotoxic activities from the endophytic fungus Annulohypoxylon squamulosum. Phytochem. Lett. 5, 219-223. doi: 10.1016/j.phytol.2011.12.012

Chinworrungsee, M., Wiyakrutta, S., Sriubolmas, N., Chuailua, P., and Suksamrarn, A. (2008). Cytotoxic activities of trichothecenes isolated from an endophytic fungus belonging to order Hypocreales. Arch. Pharm. Res. 31, 611-616. doi: 10.1007/s12272-001-1201-x

Chokpaiboon, S., Choodej, S., Boonyuen, N., Teerawatananond, T., and Pudhom, K. (2016). Highly oxygenated chromones from mangrove-derived endophytic fungus Rhytidhysteron rufulum. Phytochemistry 122, 172-177. doi: 10.1016/j.phytochem.2015.12.010

Chokpaiboon, S., Sommit, D., Teerawatananond, T., Muangsin, N., Bunyapaiboonsri, T., and Pudhom, K. (2010). Cytotoxic Nor-chamigrane and chamigrane endoperoxides from a Basidiomycetous fungus. J. Nat. Prod. 73, 1005-1007. doi: 10.1021/np100103j

Cortés, F., and Pastor, N. (2003). Induction of endoreduplication by topoisomerase II catalytic inhibitors. Mutagenesis 18, 105-112. doi: 10.1093/mutage/18.2.105

Cui, C. M., Li, X. M., Meng, L., Li, C. S., Huang, C. G., and Wang, B. G. (2010a). 7-Nor-ergosterolide, a pentalactone-containing norsteroid and related steroids from the marine-derived endophytic Aspergillus ochraceus EN-31. J. Nat. Prod. 73, 1780-1784. doi: 10.1021/np100386q

Cui, C.-M., Li, X.-M., Li, C.-S., Proksch, P., and Wang, B.-G. (2010b). Cytoglobosins A-G, cytochalasans from a marine-derived endophytic fungus, Chaetomium globosum QEN-14. J. Nat. Prod. 73, 729-733. doi: 10.1021/np900569t

Davis, R. A., Longden, J., Avery, V. M., and Healy, P. C. (2008). The isolation, structure determination and cytotoxicity of the new fungal 
metabolite, trichodermamide C. Bioorg. Med. Chem. Lett. 18, 2836-2839. doi: 10.1016/j.bmcl.2008.03.090

Debbab, A., Aly, A. H., Edrada-Ebel, R., Wray, V., Müller, W. E., Totzke, F., et al. (2009a). Bioactive metabolites from the endophytic fungus stemphylium globuliferum isolated from mentha pulegium. J. Nat. Prod. 72, 626-631. doi: $10.1021 / \mathrm{np} 8004997$

Debbab, A., Aly, H., Edrada-Ebel, R. A., Müller, W. E., Mosaddak, M., Hakiki, A., et al. (2009b). Bioactive secondary metabolites from the endophytic fungus Chaetomium sp. isolated from Salvia officinalis growing in Morocco. Biotechnol. Agron. Soc. Environ. 13, 229-234.

Demain, A. L., and Vaishnav, P. (2011). Natural products for cancer chemotherapy. Microb. Biotechnol. 4, 687-699. doi: 10.1111/j.1751-7915.2010.00221.x

Deng, B. W., Liu, K. H., Chen, W. Q., Ding, X. W., and Xie, X. C. (2008). Fusarium solani, Tax-3, a new endophytic taxol-producing fungus from Taxus chinensis. World J. Microbiol. Biotechnol. 25, 139-143. doi: 10.1007/s11274-008-9876-2

Deshmukh, S. K., Mishra, P. D., Kulkarni-Almeida, A., Verekar, S., Sahoo, M. R., Periyasamy, G., et al. (2009). Anti-inflammatory and anticancer activity of ergoflavin isolated from an endophytic fungus. Chem. Biodivers. 6, 784-789. doi: 10.1002/cbdv.200800103

Devari, S., Jaglan, S., Kumar, M., Deshidi, R., Guru, S., Bhushan, S., et al. (2014). Capsaicin production by Alternaria alternata, an endophytic fungus from Capsicum annum; LC-ESI-MS/MS analysis. Phytochemistry 98(Suppl. C), 183-189. doi: 10.1016/j.phytochem.2013.12.001

Ding, G., Zheng, Z., Liu, S., Zhang, H., Guo, L., and Che, Y. (2009). Photinides A-F, cytotoxic benzofuranone-derived $\gamma$-lactones from the plant endophytic fungus Pestalotiopsis photiniae. J. Nat. Prod. 72, 942-945. doi: 10.1021/np900084d

Ding, X., Liu, K., Deng, B., Chen, W., Li, W., and Liu, F. (2013). Isolation and characterization of endophytic fungi from Camptotheca acuminata. World J. Microbiol. Biotechnol. 29, 1831-1838. doi: 10.1007/s11274-013-1345-x

Downing, K. H. (2000). Structural basis for the interaction of tubulin with proteins and drugs that affect microtubule dynamics. Annu. Rev. Cell Dev. Biol. 16, 89-111. doi: 10.1146/annurev.cellbio.16.1.89

Dumontet, C., and Jordan, M. A. (2010). Microtubule-binding agents: a dynamic field of cancer therapeutics. Nat. Rev. Drug Discov. 9, 790-803. doi: $10.1038 / \mathrm{nrd} 3253$

Easton, H., Lyons, T., Cooper, B., and Mace, W. (2009). "Loline alkaloids for better protection of pastures from insect pests," in Proceedings of the New Zealand Grassland Association (Dunedin: New Zealand Grassland Association), 151-154.

Elavarasi, A., Rathna, G. S., and Kalaiselvam, M. (2012). Taxol producing mangrove endophytic fungi Fusarium oxysporum from Rhizophora annamalayana. Asian Pac. J. Trop. Biomed. 2, S1081-S1085. doi: 10.1016/S2221-1691(12) 60365-7

Elsebai, M. F., Tejesvi, M. V., and Pirttilä, A. M. (2014). "Endophytes as a novel source of bioactive new structures," in Advances in Endophytic Research, eds V.C. Verma and A.C. Gange (Springer), 191-202.

Eng, W. K., Faucette, L., Johnson, R. K., and Sternglanz, R. (1988). Evidence that DNA topoisomerase I is necessary for the cytotoxic effects of camptothecin. Mol. Pharmacol. 34, 755-760.

Eyberger, A. L., Dondapati, R., and Porter, J. R. (2006). Endophyte fungal isolates from Podophyllum peltatum produce podophyllotoxin. J. Nat. Prod. 69, 1121-1124. doi: 10.1021/np060174f

Fang, Z. F., Yu, S. S., Zhou, W. Q., Chen, X. G., Ma, S. G., Li, Y., et al. (2012). A new isocoumarin from metabolites of the endophytic fungus Alternaria tenuissima (Nees \& T. Nees: Fr.) Wiltshire. Chin. Chem. Lett. 23, 317-320. doi: 10.1016/j.cclet.2011.11.021

Flores-Bustamante, Z. R., Rivera-Orduña, F. N., Martínez-Cárdenas, A., and Flores-Cotera, L. B. (2010). Microbial paclitaxel: advances and perspectives. J. Antibiot. 63, 460-467. doi: 10.1038/ja.2010.83

Fouda, A. H., Hassan, S. E.-D., Eid, A. M., and Ewais, E. E.-D. (2015). Biotechnological applications of fungal endophytes associated with medicinal plant Asclepias sinaica (Bioss.). Ann. Agric. Sci. 60, 95-104. doi: 10.1016/j.aoas.2015.04.001

Froelich-Ammon, S. J., and Osheroff, N. (1995). Topoisomerase poisons: harnessing the dark side of enzyme mechanism. J. Biol. Chem. 270, 21429-21432.

Gamal-Eldeen, A. M., Abdel-Lateff, A., and Okino, T. (2009). Modulation of carcinogen metabolizing enzymes by chromanone $\mathrm{A}$; a new chromone derivative from algicolous marine fungus Penicillium sp. Environ. Toxicol. Pharmacol. 28, 317-322. doi: 10.1016/j.etap.2009.05.010

Gangadevi, V., and Muthumary, J. (2008a). Isolation of Colletotrichum gloeosporioides, a novel endophytic taxol-producing fungus from the leaves of a medicinal plant, Justicia gendarussa. Mycol. Balc. 5, 1-4.

Gangadevi, V., and Muthumary, J. (2008b). Taxol, an anticancer drug produced by an endophytic fungus Bartalinia robillardoides Tassi, isolated from a medicinal plant, Aegle marmelos Correa ex Roxb. World J. Microbiol. Biotechnol. 24:717. doi: 10.1007/s11274-007-9530-4

Gangadevi, V., and Muthumary, J. (2009a). A novel endophytic taxolproducing fungus Chaetomella raphigera isolated from a medicinal plant, Terminalia arjuna. Appl. Biochem. Biotechnol. 158, 675-684. doi: 10.1007/s12010-009-8532-0

Gangadevi, V., and Muthumary, J. (2009b). Taxol production by Pestalotiopsis terminaliae, an endophytic fungus of Terminalia arjuna (arjun tree). Biotechnol. Appl. Biochem. 52, 9-15. doi: 10.1042/BA20070243

Gangadevi, V., Murugan, M., and Muthumary, J. (2008). Taxol determination from Pestalotiopsis pauciseta, a fungal endophyte of a medicinal plant. Chin. J. Biotechnol. 24, 1433-1438. doi: 10.1016/S1872-2075(08)60065-5

Gao, N., Shang, Z.-C., Yu, P., Luo, J., Jian, K.-L., Kong, L.-Y., et al. (2017). Alkaloids from the endophytic fungus Penicillium brefeldianum and their cytotoxic activities. Chin. Chem. Lett. 28, 1194-1199. doi: 10.1016/j.cclet.2017.02.022

Garyali, S., Kumar, A., and Reddy, M. S. (2013). Taxol Production by an endophytic fungus, Fusarium redolens, isolated from Himalayan Yew. J. Microbiol. Biotechnol. 23, 1372-1380. doi: 10.4014/jmb.1305.05070

Global Burden of Disease Cancer, C. (2015). The global burden of cancer 2013. JAMA Oncol. 1, 505-527. doi: 10.1001/jamaoncol.2015.0735

Gokul Raj, K., Manikandan, R., Arulvasu, C., and Pandi, M. (2015). Antiproliferative effect of fungal taxol extracted from Cladosporium oxysporum against human pathogenic bacteria and human colon cancer cell line HCT 15. Spectrochim. Acta A Mol. Biomol. Spectrosc. 138, 667-674. doi: 10.1016/j.saa.2014.11.036

Gordaliza, M., Castro, M. A., del Corral, J. M., and Feliciano, A. S. (2000). Antitumor properties of podophyllotoxin and related compounds. Curr. Pharm. Des. 6, 1811-1839. doi: 10.2174/1381612003398582

Gordaliza, M., Castro, M. A., García-Grávalos, M. D., Ruiz, P., Miguel del Corral J. M., and Feliciano, A. S. (1994). Antineoplastic and Antiviral activities of podophyllotoxin related lignans. Arch. Pharm. 327, 175-179. doi: 10.1002/ardp.19943270309

Gordon, J. E. (2011). Spotlights: dissecting a diterpenoid cyclase. ACS Chem. Biol. 6, 124-126. doi: 10.1021/cb200022n

Greve, H., Mohamed, I. E., Pontius, A., Kehraus, S., Gross, H., and König, G. M. (2010). Fungal metabolites: structural diversity as incentive for anticancer drug development. Phytochem. Rev. 9, 537-545. doi: 10.1007/s11101-010-9198-5

Greve, H., Schupp, P. J., Eguereva, E., Kehraus, S., Kelter, G., Maier, A., et al. (2008). Apralactone A and a new stereochemical class of curvularins from the marine-derived fungus Curvularia sp. European J. Org. Chem. 2008, 5085-5092. doi: 10.1002/ejoc.200800522

Guo, B. H., Wang, Y., Zhou, X., Hu, K., Tan, F., Miao, Z., et al. (2006). An endophytic taxol-producing fungus BT2 isolated from Taxus chinensis var. mairei. Afr. J. Biotechnol. 5, 875-877.

Guo, B., Li, H., and Zhang, L. (1998). Isolation of a fungus producing vinblastine. J. Yunnan Univ. 20, 214-215.

Hande, K. (1998). Etoposide: four decades of development of a topoisomerase II inhibitor. Eur. J. Cancer 34, 1514-1521.

Hardoim, P. R., van Overbeek, L. S., and Elsas, J. D. (2008). Properties of bacterial endophytes and their proposed role in plant growth. Trends Microbiol. 16, 463-471. doi: 10.1016/j.tim.2008.07.008

Heinig, U., and Jennewein, S. (2009). Taxol: A complex diterpenoid natural product with an evolutionarily obscure origin. Afr. J. Biotechnol. 8, 1370-1385. doi: 10.5897/AJB2009.000-9217

Hemamalini, V., Kumar, D. M., Rebecca, A. I. N., Srimathi, S., Muthumary, J., and Kalaichelvan, P. (2015). Isolation and characterization of taxol producing endophytic Phoma sp. from Calotropis gigantea and its anti-proliferative studies. J. Acad. Ind. Res. 3, 645-649.

Horwitz, S. B., Lothstein, L., Manfredi, J. J., Mellado, W., Parness, J., Roy, S. N., et al. (1986). Taxol: mechanisms of action and resistance. Ann. N. Y. Acad. Sci. $466,733-744$. 
Hu, K., Tan, F., Tang, K., Zhu, S., and Wang, W. (2006). Isolation and screening of endophytic fungi synthesizing taxol from Taxus chinensis var. mairei. J. Southwest China Norm. Univ. 31, 134-137.

Huang, C. H., Pan, J. H., Chen, B., Yu, M., Huang, H. B., Zhu, X., et al. (2011). Three bianthraquinone derivatives from the mangrove endophytic fungus Alternaria sp. ZJ9-6B from the South China Sea. Mar. Drugs 9, 832-843. doi: $10.3390 / \mathrm{md} 9050832$

Huang, C., Jin, H., Song, B., Zhu, X., Zhao, H., Cai, J., et al. (2012). The cytotoxicity and anticancer mechanisms of alterporriol L, a marine bianthraquinone, against MCF-7 human breast cancer cells. Appl. Microbiol. Biotechnol. 93, 777-785. doi: 10.1007/s00253-011-3463-4

Huang, J.-X., Zhang, J., Zhang, X.-R., Zhang, K., Zhang, X., and He, X.R. (2014). Mucor fragilis as a novel source of the key pharmaceutical agents podophyllotoxin and kaempferol. Pharm. Biol. 52, 1237-1243. doi: 10.3109/13880209.2014.885061

Huang, Z., Guo, Z., Yang, R., Yin, X., Li, X., Luo, W., et al. (2009). Chemistry and cytotoxic activities of polyketides produced by the mangrove endophytic fungus Phomopsis sp. ZSU-H76. Chem. Nat. Comp. 45:625. doi: 10.1007/s10600-009-9446-3

Huang, Z., Yang, J., She, Z., and Lin, Y. (2010). Isoflavones from the mangrove endophytic fungus Fusarium sp. (ZZF41). Nat. Prod. Commun. 5, 1771-1773.

Isaka, M., Chinthanom, P., Boonruangprapa, T., Rungjindamai, N., and Pinruan, U. (2010). Eremophilane-type sesquiterpenes from the fungus Xylaria sp. BCC 21097. J. Nat. Prod. 73, 683-687. doi: 10.1021/np100030x

Isaka, M., Jaturapat, A., Rukseree, K., Danwisetkanjana, K., Tanticharoen, M., and Thebtaranonth, Y. (2001). Phomoxanthones A and B, novel xanthone dimers from the endophytic fungus Phomopsis species. J. Nat. Prod. 64, 1015-1018. doi: 10.1021/np010006h

Isaka, M., Palasarn, S., Lapanun, S., Chanthaket, R., Boonyuen, N., and Lumyong, S. (2009). Gamma-lactones and ent-eudesmane sesquiterpenes from the endophytic fungus Eutypella sp. BCC 13199. J. Nat. Prod. 72, 1720-1722. doi: $10.1021 / \mathrm{np} 900316 \mathrm{x}$

Jalgaonwala, R. E., Mohite, B. V., and Mahajan, R. T. (2017). A review: natural products from plant associated endophytic fungi. J. Microbiol. Biotechnol. Res. $1,21-32$.

Jia, M., Chen, L., Xin, H.-L., Zheng, C.-J., Rahman, K., Han, T., et al. (2016). A friendly relationship between endophytic fungi and medicinal plants: a systematic review. Front. Microbiol. 7:906. doi: 10.3389/fmicb.2016.00906

Johnson, I. S., Armstrong, J. G., Gorman, M., and Burnett, J. P. Jr. (1963). The vinca alkaloids: a new class of oncolytic agents. Cancer Res. 23, 1390-1427.

Jordan, M. A., Thrower, D., and Wilson, L. (1991). Mechanism of inhibition of cell proliferation by Vinca alkaloids. Cancer Res. 51, 2212-2222.

Joseph, B., and Priya, R. M. (2011). Bioactive compounds from endophytes and their potential in pharmaceutical effect: a review. Am. J. Biochem. Mol. Biol. 1, 291-309. doi: 10.3923/ajbmb.2011.291.309

Kai, G., Wu, C., Gen, L., Zhang, L., Cui, L., and Ni, X. (2015). Biosynthesis and biotechnological production of anti-cancer drug Camptothecin. Phytochem. Rev. 14, 525-539 doi: 10.1007/s11101-015-9405-5

Kalaiselvam, M. (2015). "Marine fungal diversity and bioprospecting," in Springer Handbook of Marine Biotechnology, ed. S.-K. Kim (Berlin; Heidelberg: Springer), 13-25.

Kasaei, A., Mobini-Dehkordi, M., Mahjoubi, F., and Saffar, B. (2017). Isolation of taxol-producing endophytic fungi from Iranian yew through novel molecular approach and their effects on human breast cancer cell line. Curr. Microbiol. 74, 702-709. doi: 10.1007/s00284-017-1231-0

Kaufmann, S. H. (1989). Induction of endonucleolytic DNA cleavage in human acute myelogenous leukemia cells by etoposide, camptothecin, and other cytotoxic anticancer drugs: a cautionary note. Cancer Res. 49, 5870-5878.

Kaur, R., Kaur, G., Gill, R. K., Soni, R., and Bariwal, J. (2014). Recent developments in tubulin polymerization inhibitors: an overview. Eur. J. Med. Chem. 87, 89-124. doi: 10.1016/j.ejmech.2014.09.051

Keerthy, H. K., Mohan, C. D., Sivaraman Siveen, K., Fuchs, J. E., Rangappa, S., Sundaram, M. S., et al. (2014). Novel synthetic biscoumarins target tumor necrosis factor-alpha in hepatocellular carcinoma in vitro and in vivo. J. Biol. Chem. 289, 31879-31890. doi: 10.1074/jbc.M114.593855

Kimura, T., Nishida, M., Kuramochi, K., Sugawara, F., Yoshida, H., and Mizushina, Y. (2008). Novel azaphilones, kasanosins A and B, which are specific inhibitors of eukaryotic DNA polymerases beta and lambda from Talaromyces sp. Bioorg. Med. Chem. 16, 4594-4599. doi: 10.1016/j.bmc.2008.02.037
Kirschner, M. W. (1980). Implications of treadmilling for the stability and polarity of actin and tubulin polymers in vivo. J. Cell Biol. 86, 330-334.

Kour, A., Shawl, A. S., Rehman, S., Sultan, P., Qazi, P. H., Suden, P., et al. (2008). Isolation and identification of an endophytic strain of Fusarium oxysporum producing podophyllotoxin from Juniperus recurva. World J. Microbiol. Biotechnol. 24, 1115-1121. doi: 10.1007/s11274-0079582-5

Kumar, A., Patil, D., Rajamohanan, P. R., and Ahmad, A. (2013). Isolation, purification and characterization of vinblastine and vincristine from endophytic fungus Fusarium oxysporum isolated from Catharanthus roseus. PLoS ONE 8:e71805. doi: 10.1371/journal.pone.0071805.

Kumaran, R. S., and Hur, B. K. (2009). Screening of species of the endophytic fungus Phomopsis for the production of the anticancer drug taxol. Biotechnol. Appl. Biochem. 54, 21-30. doi: 10.1042/BA20080110

Kumaran, R. S., Choi, Y.-K., Lee, S., Jeon, H. J., Jung, H., and Kim, H. J. (2012). Isolation of taxol, an anticancer drug produced by the endophytic fungus, Phoma betae. Afr. J. Biotechnol. 11, 950-960. doi: 10.5897/AJB11.1937

Kumaran, R. S., Muthumary, J., and Hur, B.-K. (2008a). Isolation and identification of taxol, an anticancer drug from Phyllosticta melochiae yates, an endophytic fungus of Melochia corchorifolia L. Food Sci. Biotechnol. 17, 1246-1253.

Kumaran, R. S., Muthumary, J., and Hur, B.-K. (2008b). Taxol from Phyllosticta citricarpa, a leaf spot fungus of the angiosperm Citrus medica. J. Biosci. Bioeng. 106, 103-106. doi: 10.1263/jbb.106.103

Kumaran, R. S., Muthumary, J., Kim, E.-K., and Hur, B.-K. (2009). Production of taxol from Phyllosticta dioscoreae, a leaf spot fungus isolated from Hibiscus rosasinensis. Biotechnol. Bioprocess Eng.14, 76-83. doi: 10.1007/s12257-008-0041-4

Kusari, S., and Spiteller, M. (2012). "Metabolomics of endophytic fungi producing associated plant secondary metabolites: progress, challenges and opportunities," in Metabolomics, ed U. Roessner (Rijeka: InTech), 241-266.

Kusari, S., Hertweck, C., and Spiteller, M. (2012). Chemical ecology of endophytic fungi: origins of secondary metabolites. Chem. Biol. 19, 792-798. doi: 10.1016/j.chembiol.2012.06.004

Kusari, S., Lamshoft, M., and Spiteller, M. (2009a). Aspergillus fumigatus Fresenius, an endophytic fungus from Juniperus communis L. Horstmann as a novel source of the anticancer pro-drug deoxypodophyllotoxin. J. Appl. Microbiol. 107, 1019-1030. doi: 10.1111/j.1365-2672.2009.04285.x

Kusari, S., Singh, S., and Jayabaskaran, C. (2014). Rethinking production of Taxol(R) (paclitaxel) using endophyte biotechnology. Trends Biotechnol. 32, 304-311. doi: 10.1016/j.tibtech.2014.03.011

Kusari, S., Zühlke, S., and Spiteller, M. (2009b). An endophytic fungus from Camptotheca acuminata that produces camptothecin and analogues. J. Nat. Prod. 72, 2-7. doi: 10.1021/np800455b

Lee, Y. M., Li, H., Hong, J., Cho, H. Y., Bae, K. S., Kim, M. A., et al. (2010). Bioactive metabolites from the sponge-derived fungus Aspergillus versicolor. Arch. Pharm. Res. 33, 231-235. doi: 10.1007/s12272-010-0207-4

Lei, M., Ribeiro, H., Kolodin, G., Gill, J., Wang, Y. S., Maloney, D., et al. (2013). Establishing a high-throughput and automated cancer cell proliferation panel for oncology lead optimization. J. Biomol. Screen. 18, 1043-1053. doi: $10.1177 / 1087057113491825$

Li, C.-T., Li, Y., Wang, Q.-J., and Sung, C.-K. (2008). Taxol production by Fusarium arthrosporioides isolated from yew, Taxus cuspidata. J. Med. Biochem. 27, 454-458. doi: 10.2478/v10011-008-0022-3

Li, G., and Kusari, S. (2017). Epigenetic modulation of endophytic Eupenicillium sp. LG41 by a histone deacetylase inhibitor for production of decalin-containing compounds. J. Nat. Prod. 80, 983-988. doi: 10.1021/acs.jnatprod.6b00997

Li, J. Y. (1996). Endophytic taxol-producing fungi from bald cypress, Taxodium distichum. Microbiology 142, 2223-2226. doi: 10.1099/13500872-142-8-2223

Li, J., Sun, H., Jin, L., Cao, W., Zhang, J., Guo, C.-Y., et al. (2013). Alleviation of podophyllotoxin toxicity using coexisting flavonoids from Dysosma versipellis. PLoS ONE 8:e72099. doi: 10.1371/journal.pone.0072099

Li, J.-Y. (1998). The induction of taxol production in the endophytic fungus Periconia sp. from Torreya grandifolia. J. Ind. Microbiol. 20, 259-264. doi: 10.1038/sj.jim.2900521

Li, K. K., Lu, Y. J., Song, X. H., She, Z. G., Wu, X. W., An, L. K., et al. (2010). The metabolites of mangrove endophytic fungus Zh6-B1 from the South China Sea. Bioorg. Med. Chem. Lett. 20, 3326-3328. doi: 10.1016/j.bmcl.2010.04.036

Liang, Z., Zhang, J., Zhang, X., Li, J., Zhang, X., and Zhao, C. (2016). Endophytic fungus from Sinopodophyllum emodi (Wall.) ying 
that produces podophyllotoxin. J. Chromatogr. Sci. 54, 175-178. doi: 10.1093/chromsci/bmv124

Lin, T., Wang, G. H., Lin, X., Hu, Z. Y., Chen, Q. C., Xu, Y., et al. (2011). Three new oblongolides from Phomopsis sp. XZ-01, an endophytic fungus from Camptotheca acuminate. Molecules 16, 3351-3359. doi: 10.3390/molecules 16043351

Lin, T., Wang, G., Zeng, D., and Chen, H. (2015). Cytotoxic metabolites from Botryotinia fuckeliana A-S-3: an endophytic fungus from Ajuga decumbens. Phytochem. Lett. 13(Suppl C), 206-211. doi: 10.1016/j.phytol.2015.06.007

Liu, K., Ding, X., Deng, B., and Chen, W. (2009). Isolation and characterization of endophytic taxol-producing fungi from Taxus chinensis. J. Ind. Microbiol. Biotechnol. 36, 1171-1177. doi: 10.1007/s10295-009-0598-8

Liu, L., Liu, S., Niu, S., Guo, L., Chen, X., and Che, Y. (2009). Isoprenylated chromone derivatives from the plant endophytic fungus Pestalotiopsis fici. J. Nat. Prod. 72, 1482-1486. doi: 10.1021/np900308s

Lu, Z., Zhu, H., Fu, P., Wang, Y., Zhang, Z., Lin, H., et al. (2010). Cytotoxic polyphenols from the marine-derived fungus Penicillium expansum. J. Nat. Prod. 73, 911-914. doi: 10.1021/np100059m.

Majumder, A., and Jha, S. (2009). Biotechnological approaches for the production of potential anticancer leads podophyllotoxin and paclitaxel: an overview. J. Biol. Sci. 1, 46-69.

Manfredi, J. J., Parness, J., and Horwitz, S. B. (1982). Taxol binds to cellular microtubules. J. Cell Biol. 94, 688-696. doi: 10.1083/jcb.94.3.688

Merlin, J. N., Christhudas, I. N., Kumar, P. P., Kumar, M., and Agastian, P. (2012). Taxol production by endophytic Fusarium solani LCPANCF01 from Tylophora indica. J. Acad. Indus. Res. 1:281.

Miyasaka, T., Sawada, S., Nokata, K., Sugino, E., and Mutai, M. (1986). Camptothecin Derivatives and Process for Preparing Same. Tokyo: Google Patents.

Mohamed, I. E., Gross, H., Pontius, A., Kehraus, S., Krick, A., Kelter, G., et al. (2009). Epoxyphomalin A and B, prenylated polyketides with potent cytotoxicity from the marine-derived fungus Phoma sp. Org. Lett. 11, 5014-5017. doi: 10.1021/ol901996g

Mohamed, S., Mahmoud, M., and El Sayed, O. (2013). Antioxidant and antitumor activity of a new sesquiterpene isolated from endophytic fungus Aspergillus glaucus. Int. J. Pharm.Tech. Res. 5:391.

Mohan, C. D., Bharathkumar, H., Bulusu, K. C., Pandey, V., Rangappa, S., Fuchs, J. E., et al. (2014). Development of a novel azaspirane that targets the Janus kinase-signal transducer and activator of transcription (STAT) pathway in hepatocellular carcinoma in vitro and in vivo. J. Biol. Chem. 289, 34296-34307. doi: $10.1074 /$ jbc.M114.601104

Mohan, C. D., Srinivasa, V., Rangappa, S., Mervin, L., Mohan, S., Paricharak, S., et al. (2016). Trisubstituted-Imidazoles induce apoptosis in human breast cancer cells by targeting the oncogenic PI3K/Akt/mTOR signaling pathway. PLoS ONE 11:e0153155. doi: 10.1371/journal.pone.0153155

Mohana Kumara, P., Zuehlke, S., Priti, V., Ramesha, B. T., Shweta, S., Ravikanth, G., et al. (2012). Fusarium proliferatum, an endophytic fungus from dysoxylum Binectariferum Hook.f, produces rohitukine, a chromane alkaloid possessing anti-cancer activity. Antonie Van Leeuwenhoek 101, 323-329. doi: $10.1007 / \mathrm{s} 10482-011-9638-2$

Moricca, S., and Ragazzi, A. (2008). Fungal endophytes in Mediterranean oak forests: a lesson from Discula quercina. Phytopathology 98, 380-386. doi: 10.1094/phyto-98-4-0380

Moudi, M., Go, R., Yien, C. Y. S., and Nazre, M. (2013). Vinca alkaloids. Int. J. Prev. Med. 4, 1231-1235.

Nadeem, M., Ram, M., Alam, P., Ahmad, M. M., Mohammad, A., Al-Qurainy, F., et al. (2012). Fusarium solani, P1, a new endophytic podophyllotoxinproducing fungus from roots of Podophyllum hexandrum. Afr. J. Microbiol. Res. 6, 2493-2499. doi: 10.5897/AJMR11.1596

Nadeem, M., Rikhari, H. C., Kumar, A., Palni, L. M., and Nandi, S. K. (2002). Taxol content in the bark of Himalayan Yew in relation to tree age and sex. Phytochemistry 60, 627-631. doi: 10.1016/S0031-9422(02)00115-2

Neelgundmath, M., Dinesh, K. R., Mohan, C. D., Li, F., Dai, X., Siveen, K. S., et al. (2015). Novel synthetic coumarins that targets NF-kappaB in Hepatocellular carcinoma. Bioorg. Med. Chem. Lett. 25, 893-897. doi: 10.1016/j.bmcl.2014.12.065

Newman, D. J., and Cragg, G. M. (2007). Natural products as sources of new drugs over the last 25 years. J. Nat. Prod. 70, 461-477. doi: 10.1021/np068054v
Nirvanappa, A. C., Mohan, C. D., Rangappa, S., Ananda, H., Sukhorukov, A. Y., Shanmugam, M. K., et al. (2016). Novel synthetic oxazines target NF-kappaB in colon cancer in vitro and inflammatory bowel disease in vivo. PLoS ONE 11:e0163209. doi: 10.1371/journal.pone.0163209

Nithya, K., and Muthumary, J. (2009). Growth studies of Colletotrichum gloeosporioides (Penz.) Sacc.-a taxol producing endophytic fungus from Plumeria acutifolia. Indian J. Sci. Technol. 2, 14-19. doi: $10.17485 /$ ijst/2009/v2i11/29528

Nitiss, J. L. (2009). Targeting DNA topoisomerase II in cancer chemotherapy. Nat. Rev. Cancer 9, 338-350. doi: 10.1038/nrc2607

Noble, R. (2016). Anti-cancer alkaloids of Vinca rosea. Pharmacol. Orient. Plants 7 , 61-78.

Noble, R. L. (1990). The discovery of the vinca alkaloids-chemotherapeutic agents against cancer. Biochem. Cell Biol. 68, 1344-1351. doi: 10.1139/o90-197

Ochoa-Villarreal, M., Howat, S., Hong, S., Jang, M. O., Jin, Y.-W., Lee, E.-K., et al. (2016). Plant cell culture strategies for the production of natural products. $B M B$ Rep. 49, 149-158. doi: 10.5483/BMBRep.2016.49.3.264

Palem, P. P., Kuriakose, G. C., and Jayabaskaran, C. (2015). An endophytic fungus, Talaromyces radicus, isolated from Catharanthus roseus, produces vincristine and vinblastine, which induce apoptotic cell death. PLOS ONE 10:e0144476. doi: 10.1371/journal.pone.0144476

Pandi, M., Kumaran, R. S., Choi, Y.-K., Kim, H. J., and Muthumary, J. (2011). Isolation and detection of taxol, an anticancer drug produced from Lasiodiplodia theobromae, an endophytic fungus of the medicinal plant Morinda citrifolia. Afr. J. Biotechnol. 10, 1428-1435.

Parasuraman, S. (2011). Toxicological screening. J. Pharmacol. Pharmacother. 2, 74-79. doi: 10.4103/0976-500X.81895

Pendleton, M., Lindsey, R. H., Felix, C. A., Grimwade, D., and Osheroff, N. (2014). Topoisomerase II and leukemia. Ann. N. Y. Acad. Sci. 1310, 98-110. doi: $10.1111 /$ nyas. 12358

Pommier, Y. (2006). Topoisomerase I inhibitors: camptothecins and beyond. Nat. Rev. Cancer 6, 789-802. doi: 10.1038/nrc1977

Pompeng, P., Sommit, D., Sriubolmas, N., Ngamrojanavanich, N., Matsubara, K., and Pudhom, K. (2013). Antiangiogenetic effects of anthranoids from Alternaria sp., an endophytic fungus in a Thai medicinal plant Erythrina variegata. Phytomedicine 20, 918-922. doi: 10.1016/j.phymed.2013.03.019

Prasad, V., De Jesús, K., and Mailankody, S. (2017). The high price of anticancer drugs: origins, implications, barriers, solutions. Nat. Rev. Clin. Oncol. 14, 381-390. doi: 10.1038/nrclinonc.2017.31

Pu, X., Qu, X., Chen, F., Bao, J., Zhang, G., and Luo, Y. (2013). Camptothecin-producing endophytic fungus Trichoderma atroviride LY357: isolation, identification, and fermentation conditions optimization for camptothecin production. Appl. Microbiol. Biotechnol. 97, 9365-9375. doi: 10.1007/s00253-013-5163-8

Puri, S. C., Nazir, A., Chawla, R., Arora, R., Riyaz-Ul-Hasan, S., Amna, T., et al. (2006). The endophytic fungus Trametes hirsuta as a novel alternative source of podophyllotoxin and related aryl tetralin lignans. J. Biotechnol. 122, 494-510. doi: 10.1016/j.jbiotec.2005.10.015

Puri, S. C., Verma, V., Amna, T., Qazi, G. N., and Spiteller, M. (2005). An endophytic fungus from Nothapodytes foetida that produces camptothecin. $J$. Nat. Prod. 68, 1717-1719. doi: 10.1021/np0502802

Qaderi, A., Omidi, M., Etminan, A., Oladzad, A., Ebrahimi, C., Dehghani, M. M., et al. (2012). Hazel (Corylus avellana L.) as a new source of taxol and taxanes. J. Med. Plants 11, 66-77.

Qiao, W., Ling, F., Yu, L., Huang, Y., and Wang, T. (2017). Enhancing taxol production in a novel endophytic fungus, Aspergillus aculeatinus Tax6, isolated from Taxus chinensis var. mairei. Fungal Biol. 121, 1037-1044. doi: 10.1016/j.funbio.2017.08.011

Qin, J. C., Zhang, Y. M., Gao, J. M., Bai, M. S., Yang, S. X., Laatsch, H., et al. (2009). Bioactive metabolites produced by Chaetomium globosum, an endophytic fungus isolated from Ginkgo biloba. Bioorg. Med. Chem. Lett. 19, 1572-1574. doi: 10.1016/j.bmcl.2009.02.025

Raghukumar, C. (2008). Marine fungal biotechnology: an ecological perspective. Fungal Divers. 31, 19-36.

Rai, M., Rathod, D., Agarkar, G., Dar, M., Brestic, M., Pastore, G. M., et al. (2014). Fungal growth promotor endophytes: a pragmatic approach towards sustainable food and agriculture. Symbiosis 62, 63-79. doi: $10.1007 /$ s13199-014-0273-3 
Rakesh, K. S., Jagadish, S., Vinayaka, A. C., Hemshekhar, M., Paul, M., Thushara, R. M., et al. (2014). A new ibuprofen derivative inhibits platelet aggregation and ROS mediated platelet apoptosis. PLOS ONE 9:e107182. doi: 10.1371/journal.pone. 0107182

Ramesha, A., Venkataramana, M., Nirmaladevi, D., Gupta, V. K., Chandranayaka, S., and Srinivas, C. (2015). Cytotoxic effects of oosporein isolated from endophytic fungus Cochliobolus kusanoi. Front. Microbiol. 6:870. doi: $10.3389 /$ fmicb.2015.00870

Ran, X., Zhang, G., Li, S., and Wang, J. (2017). Characterization and antitumor activity of camptothecin from endophytic fungus Fusarium solani isolated from Camptotheca acuminate. Afr. Health Sci. 17, 566-574. doi: 10.4314/ahs.v17i2.34

Rateb, M. E., and Ebel, R. (2011). Secondary metabolites of fungi from marine habitats. Nat. Prod. Rep. 28, 290-344. doi: 10.1039/c0np00061b

Rebecca, A. I. N., Hemamalini, V., Kumar, D. M., Srimathi, S., Muthumary, J., and Kalaichelvan, P. (2012). Endophytic Chaetomium sp. from Michelia champaca L. and its taxol production. J. Acad. Ind. Res. 1, 68-72.

Rehman, S., Shawl, A. S., Kour, A., Andrabi, R., Sudan, P., Sultan, P., et al. (2008). An endophytic Neurospora sp. from Nothapodytes foetida producing camptothecin. Appl. Biochem. Microbiol. 44, 203-209. doi: $10.1134 / \mathrm{s} 0003683808020130$

Remesh, A. (2017). Toxicities of anticancer drugs and its management. Int. J. Basic Clin. Pharmacol. 1, 2-12. doi: 10.5455/2319-2003.ijbcp000812

Roopa, G., Madhusudhan, M. C., Sunil, K. C. R., Lisa, N., Calvin, R., Poornima, R., et al. (2015). Identification of Taxol-producing endophytic fungi isolated from Salacia oblonga through genomic mining approach. J. Genet. Eng. Biotechnol. 13, 119-127. doi: 10.1016/j.jgeb.2015.09.002

Roopashree, R., Mohan, C. D., Swaroop, T. R., Jagadish, S., Raghava, B., Balaji, K. S., et al. (2015). Novel synthetic bisbenzimidazole that targets angiogenesis in Ehrlich ascites carcinoma bearing mice. Bioorg. Med. Chem. Lett. 25, 2589-2593. doi: 10.1016/j.bmcl.2015.04.010

Salvador-Reyes, L. A., and Luesch, H. (2015). Biological targets and mechanisms of action of natural products from marine cyanobacteria. Nat. Prod. Rep. 32, 478-503. doi: 10.1039/C4NP00104D

Schiff, P. B., Fant, J., and Horwitz, S. B. (1979). Promotion of microtubule assembly in vitro by taxol. Nature 277:665. doi: 10.1038/277665a 0

Schulz, B., Boyle, C., Draeger, S., RÖMmert, A.-K., and Krohn, K. (2002). Endophytic fungi: a source of novel biologically active secondary metabolites. Mycol. Res. 106, 996-1004. doi: 10.1017/S0953756202006342

Schulz, B., Römmert, A.-K., Dammann, U., Aust, H.-J., and Strack, D. (1999). The endophyte-host interaction: a balanced antagonism? Mycol. Res. 103, 1275-1283. doi: 10.1017/S0953756299008540

Sebastian, A., Pandey, V., Mohan, C. D., Chia, Y. T., Rangappa, S., Mathai, J., et al. (2016). Novel adamantanyl-based thiadiazolyl pyrazoles targeting EGFR in triple-negative breast cancer. ACS Omega 1, 1412-1424. doi: 10.1021/acsomega.6b00251

Senthil Kumaran, R., Muthumary, J., and Hur, B. (2008). Production of Taxol from Phyllosticta spinarum, an endophytic fungus of Cupressus sp. Eng. Life Sci. 8, 438-446. doi: 10.1002/elsc.200800019

Shao, C.-L., Wang, C.-Y., Gu, Y.-C., Wei, M.-Y., Pan, J.-H., Deng, D.-S., et al. (2010). Penicinoline, a new pyrrolyl 4-quinolinone alkaloid with an unprecedented ring system from an endophytic fungus Penicillium sp. Bioorg. Med. Chem. Lett. 20, 3284-3286. doi: 10.1016/j.bmcl.2010.04.043

Shiono, Y., Yokoi, M., Koseki, T., Murayama, T., Aburai, N., and Kimura, K. (2010). Allantopyrone A, a new alpha-pyrone metabolite with potent cytotoxicity from an endophytic fungus, Allantophomopsis lycopodina KS-97. J. Antibiot. 63, 251-253. doi: 10.1038/ja.2010.18

Shweta, S., Gurumurthy, B. R., Ravikanth, G., Ramanan, U. S., and Shivanna, M. B. (2013). Endophytic fungi from Miquelia dentata Bedd., produce the anti-cancer alkaloid, camptothecine. Phytomedicine 20, 337-342. doi: 10.1016/j.phymed.2012.11.015

Shweta, S., Zuehlke, S., Ramesha, B. T., Priti, V., Mohana Kumar, P., Ravikanth, G., et al. (2010). Endophytic fungal strains of Fusarium solani, from Apodytes dimidiata E. Mey. ex Arn (Icacinaceae) produce camptothecin, 10-hydroxycamptothecin and 9-methoxycamptothecin. Phytochemistry 71, 117-122. doi: 10.1016/j.phytochem.2009.09.030

Siegel, R. L., Miller, K. D., and Jemal, A. (2017). Cancer Statistics, 2017. CA Cancer J. Clin. 67, 7-30. doi: 10.3322/caac.21387
Singh, S., Sharma, B., Kanwar, S. S., and Kumar, A. (2016). Lead phytochemicals for anticancer drug development. Front. Plant Sci. 7:1667. doi: $10.3389 /$ fpls.2016.01667

Soliman, S. S., Tsao, R., and Raizada, M. N. (2011). Chemical inhibitors suggest endophytic fungal paclitaxel is derived from both mevalonate and non-mevalonate-like pathways. J. Nat. Prod. 74, 2497-2504. doi: $10.1021 / \mathrm{np} 200303 \mathrm{v}$

Somjaipeng, S., Medina, A., and Magan, N. (2016). Environmental stress and elicitors enhance taxol production by endophytic strains of Paraconiothyrium variabile and Epicoccum nigrum. Enzyme Microb. Technol. 90, 69-75. doi: 10.1016/j.enzmictec.2016.05.002

Srinivas, V., Mohan, C. D., Baburajeev, C. P., Rangappa, S., Jagadish, S., Fuchs, J. E., et al. (2015). Synthesis and characterization of novel oxazines and demonstration that they specifically target cyclooxygenase 2. Bioorg. Med. Chem. Lett. 25, 2931-2936. doi: 10.1016/j.bmcl.2015.05.047

Stierle, A. A., and Stierle, D. B. (2015). Bioactive secondary metabolites produced by the fungal endophytes of conifers. Nat. Prod. Commun. 10, 1671-1682.

Stierle, A., Strobel, G., and Stierle, D. (1993). Taxol and taxane production by Taxomyces andreanae, an endophytic fungus of Pacific yew. Science 260, 214-216. doi: 10.1126/science.8097061

Strobel, G. A. (2003). Endophytes as sources of bioactive products. Microbes Infect. 5, 535-544. doi: 10.1016/S1286-4579(03)00073-X

Strobel, G. A., Hess, W. M., and Li, J. Y. (1997). Pestalotiopsis guepinii, a taxol producing endophyte of the Wollemi pine, Wollemia nobilis. Aust. J. Bot. 45, 1073-1082. doi: 10.1071/bt96094

Strobel, G., Daisy, B., Castillo, U., and Harper, J. (2004). Natural products from endophytic microorganisms. J. Nat. Prod. 67, 257-268. doi: 10.1021/np030397v

Strobel, G., Yang, X., Sears, J., Kramer, R., Sidhu, R. S., and Hess, W. (1996). Taxol from Pestalotiopsis microspora, an endophytic fungus of Taxus wallachiana. Microbiology 142, 435-440. doi: 10.1099/13500872-142-2-435

Su, H., Kang, J., Cao, J., Mo, L., and Hyde, K. D. (2014). Medicinal plant endophytes produce analogous bioactive compounds. Chiang Mai J. Sci. 41, 1-13.

Sun, D., Ran, X., and Wang, J. (2008). Isolation and identification of a taxolproducing endophytic fungus from Podocarpus. Wei Sheng Wu Xue Bao 48, 589-595.

Sun, X., Kong, X., Gao, H., Zhu, T., Wu, G., Gu, Q., et al. (2014). Two new meroterpenoids produced by the endophytic fungus Penicillium sp. SXH-65. Arch. Pharm. Res. 37, 978-982. doi: 10.1007/s12272-013-0268-2

Sun, Z. L., Zhang, M., Zhang, J. F., and Feng, J. (2011). Antifungal and cytotoxic activities of the secondary metabolites from endophytic fungus Massrison sp. Phytomedicine 18, 859-862. doi: 10.1016/j.phymed.2011.01.019

Tabata, H. (2006). Production of paclitaxel and the related taxanes by cell suspension cultures of Taxus species. Curr. Drug Targets 7, 453-461. doi: $10.2174 / 138945006776359368$

Takimoto, C. H. (2002). "Camptothecins A2 - Bertino, Joseph R," in Encyclopedia of Cancer, 2nd Edn., ed J. Bertino (New York, NY: Academic Press), 301-309.

Tulp, M., and Bohlin, L. (2002). Functional versus chemical diversity: is biodiversity important for drug discovery? Trends Pharmacol. Sci. 23, 225-231. doi: 10.1016/S0165-6147(02)02007-2

Ulukan, H., and Swaan, P. W. (2002). Camptothecins. Drugs 62, 2039-2057. doi: 10.2165/00003495-200262140-00004

Van Maanen, J., Retèl, J., De Vries, J., and Pinedo, H. (1988). Mechanism of action of antitumor drug etoposide: a review. J. Natl. Cancer Inst. 80, 1526-1533.

Vasundhara, M., Kumar, A., and Reddy, M. S. (2016). Molecular approaches to screen bioactive compounds from endophytic fungi. Front. Microbiol. 7:1774. doi: 10.3389/fmicb.2016.01774

Venkatachalam, R., Subban, K., and Paul, M. J. (2008). Taxol from Botryodiplodia theobromae (BT 115) - AN endophytic fungus of Taxus baccata. J. Biotechnol. 136, S189-S190. doi: 10.1016/j.jbiotec.2008.07.1823

Venugopalan, A., and Srivastava, S. (2015). Endophytes as in vitro production platforms of high value plant secondary metabolites. Biotechnol. Adv. 33(Pt. 1), 873-887. doi: 10.1016/j.biotechadv.2015.07.004

Vita-Marques, A. M., Lira, S. P., Berlinck, R. G. S., Seleghim, M. H. R., Sponchiado, S. R. P., Tauk-Tornisielo, S. M., et al. (2008). A multiscreening approach for marine-derived fungal metabolites and the isolation of cyclodepsipeptides from Beauveria felina. Química Nova 31, 1099-1103. doi: 10.1590/S0100-40422008000500030 
Wang, C., Wu, J., and Mei, X. (2001). Enhanced taxol production and release in Taxus chinensis cell suspension cultures with selected organic solvents and sucrose feeding. Biotechnol. Prog. 17, 89-94. doi: 10.1021/bp0001359

Wang, F. W., Hou, Z. M., Wang, C. R., Li, P., and Shi, D. H. (2008). Bioactive metabolites from Penicillium sp., an endophytic fungus residing in Hopea hainanensis. World J.Microbiol. Biotechnol. 24, 2143-2147. doi: 10.1007/s11274-008-9720-8

Wang, H., Liu, T., and Xin, Z. (2014). A new glucitol from an endophytic fungus Fusarium equiseti Salicorn 8. Eur. Food Res. Technol. 239, 365-376. doi: $10.1007 / \mathrm{s} 00217-014-2230-\mathrm{z}$

Wang, J., Li, G., Lu, H., Zheng, Z., Huang, Y., and Su, W. (2000). Taxol from Tubercularia sp. strain TF5, an endophytic fungus of Taxus mairei. FEMS Microbiol. Lett. 193, 249-253. doi: 10.1111/j.1574-6968.2000.tb09432.x

Wang, Q. X., Li, S. F., Zhao, F., Dai, H. Q., Bao, L., Ding, R., et al. (2011). Chemical constituents from endophytic fungus Fusarium oxysporum. Fitoterapia 82, 777-781. doi: 10.1016/j.fitote.2011.04.002

Wang, T., Ma, Y.-X., Ye, Y.-H., Zheng, H.-M., Zhang, B.-W., and Zhang, E.-H. (2017). Screening and identification of endophytic fungi producing podophyllotoxin compounds in Sinopodophyllum hexandrum stems. Chinese J. Exp.Trad. Med. Formul. 2:006.

Wang, Y., Xu, L., Ren, W., Zhao, D., Zhu, Y., and Wu, X. (2012). Bioactive metabolites from Chaetomium globosum L18, an endophytic fungus in the medicinal plant Curcuma wenyujin. Phytomedicine 19, 364-368. doi: 10.1016/j.phymed.2011.10.011

Weaver, B. A. (2014). How Taxol/paclitaxel kills cancer cells. Mol. Biol. Cell 25, 2677-2681. doi: 10.1091/mbc.E14-04-0916

Wen, L., Chen, G., She, Z., Yan, C., Cai, J., and Mu, L. (2010). Two new paeciloxocins from a mangrove endophytic fungus Paecilomyces sp. Russ. Chem. Bull. 59, 1656-1659. doi: 10.1007/s11172-010-0290-1

Wijeratne, E. M., Paranagama, P. A., Marron, M. T., Gunatilaka, M. K., Arnold, A. E., and Gunatilaka, A. A. L. (2008). Sesquiterpene quinones and related metabolites from Phyllosticta spinarum, a fungal strain endophytic in Platycladus orientalis of the Sonoran Desert. J. Nat. Prod. 71, 218-222. doi: $10.1021 / \mathrm{np} 070600 \mathrm{c}$

Wilson, L., Panda, D., and Jordan, M. A. (1999). Modulation of microtubule dynamics by drugs: a paradigm for the actions of cellular regulators. Cell Struct. Funct. 24, 329-335.

Wu, L. S., Hu, C. L., Han, T., Zheng, C. J., Ma, X. Q., Rahman, K., et al. (2013), Cytotoxic metabolites from Perenniporia tephropora, an endophytic fungus from Taxus chinensis var. mairei. Appl. Microbiol. Biotechnol. 97, 305-315. doi: 10.1007/s00253-012-4189-7

Xiao, J., Zhang, Q., Gao, Y.-Q., Tang, J.-J., Zhang, A.-L., and Gao, J.-M. (2014). Secondary metabolites from the endophytic Botryosphaeria dothidea of Melia azedarach and their antifungal, antibacterial, antioxidant, and cytotoxic activities. J. Agric. Food Chem. 62, 3584-3590. doi: 10.1021/jf500054f

Xie, S., and Zhou, J. (2017). Harnessing plant biodiversity for the discovery of novel anticancer drugs targeting microtubules. Front. Plant Sci. 8:720. doi: $10.3389 /$ fpls.2017.00720

Xu, F., Tao, W., Cheng, L., and Guo, L. (2006). Strain improvement and optimization of the media of taxol-producing fungus Fusarium maire. Biochem. Eng. J. 31, 67-73. doi: 10.1016/j.bej.2006.05.024

Xu, J., Kjer, J., Sendker, J., Wray, V., Guan, H., Edrada, R., et al. (2009a). Chromones from the endophytic fungus Pestalotiopsis sp. isolated from the chinese mangrove plant Rhizophora mucronata. J. Nat. Prod. 72, 662-665. doi: $10.1021 / \mathrm{np} 800748 \mathrm{u}$

Xu, J., Kjer, J., Sendker, J., Wray, V., Guan, H., Edrada, R., et al. (2009b). Cytosporones, coumarins, and an alkaloid from the endophytic fungus Pestalotiopsis sp. isolated from the Chinese mangrove plant Rhizophoramucronata. Bioorg. Med. Chem. 17, 7362-7367. doi: $10.1016 /$ j.bmc.2009.08.031

Yang, J., Xu, F., Huang, C., Li, J., She, Z., Pei, Z., et al. (2010). Metabolites from the mangrove endophytic fungus Phomopsis sp. (\#zsu-H76). Eur. J. Organ. Chem. 2010, 3692-3695. doi: 10.1002/ejoc.201000329

Yang, X., Zhang, L., Guo, B., and Guo, S. (2004). Preliminary study of a vincristineproudcing endophytic fungus isolated from leaves of Catharanthus roseus. Zhong Cao Yao 35, 79-81.

Yang, Y., Zhao, H., Barrero, R. A., Zhang, B., Sun, G., Wilson, I. W., et al. (2014). Genome sequencing and analysis of the paclitaxel-producing endophytic fungus Penicillium aurantiogriseum NRRL 62431. BMC Genomics 15:69. doi: 10.1186/1471-2164-15-69
Zaiyou, J., Hongsheng, W., Ning, W., Li, M., and Guifang, X. (2015). Isolation and identification of an endophytic fungus producing paclitaxel from Taxus wallichiana var mairei. Nutr. Hosp. 32, 2932-2937. doi: 10.3305/nh.2015.32.6.9781

Zaiyou, J., Li, M., and Xiqiao, H. (2017). An endophytic fungus efficiently producing paclitaxel isolated from Taxus wallichiana var. mairei. Medicine 96:e7406. doi: 10.1097/MD.0000000000007406

Zaiyou, J., Li, M., Guifang, X., and Xiuren, Z. (2013). Isolation of an endophytic fungus producing baccatin III from Taxus wallichiana var. mairei. J. Ind. Microbiol. Biotechnol. 40, 1297-1302. doi: 10.1007/s10295-013-1320-4

Zhang, J. Y., Tao, L. Y., Liang, Y. J., Yan, Y. Y., Dai, C. L., Xia, X. K., et al. (2009). Secalonic acid D induced leukemia cell apoptosis and cell cycle arrest of G(1) with involvement of GSK-3beta/beta-catenin/c-Myc pathway. Cell Cycle 8, 2444-2450. doi: 10.4161/cc.8.15.9170

Zhang, J., Ge, H. M., Jiao, R. H., Li, J., Peng, H., Wang, Y. R., et al. (2010). Cytotoxic chaetoglobosins from the endophyte Chaetomium globosum. Planta Med. 76, 1910-1914. doi: 10.1055/s-0030-1249936

Zhang, J.-Y., Tao, L.-Y., Liang, Y.-J., Chen, L.-M., Mi, Y.-J., Zheng, L.-S., et al. (2010). Anthracenedione derivatives as anticancer agents isolated from secondary metabolites of the mangrove endophytic fungi. Mar. Drugs 8, 1469-1481. doi: 10.3390/md8041469

Zhang, L., Guo, B., Li, H., Zeng, S., Shao, H., Gu, S., et al. (2000). Preliminary study on the isolation of endophytic fungus of Catharanthus roseus and its fermentation to produce products of therapeutic value. Zhong Cao Yao 31, 805-807.

Zhang, P., Zhou, P. P., and Yu, L. J. (2009). An endophytic taxol-producing fungus from Taxus media, Cladosporium cladosporioides MD2. Curr. Microbiol. 59, 227-232. doi: 10.1007/s00284-008-9270-1

Zhang, P., Zhou, P. P., Jiang, C., Yu, H., and Yu, L. J. (2008). Screening of Taxolproducing fungi based on PCR amplification from Taxus. Biotechnol. Lett. 30, 2119-2123. doi: 10.1007/s10529-008-9801-7

Zhao, J., Mou, Y., Shan, T., Li, Y., Zhou, L., Wang, M., et al. (2010a). Antimicrobial metabolites from the endophytic fungus Pichia guilliermondii isolated from Paris polyphylla var. yunnanensis. Molecules 15, 7961-7970. doi: 10.3390/molecules 15117961

Zhao, J., Zhou, L., Wang, J., Shan, T., Zhong, L., Liu, X., et al. (2010b). Endophytic fungi for producing bioactive compounds originally from their host plants. Curr. Res. Technol. Educ. Trop. Appl. Microbiol. Microb. Biotechnol. 1, 567-576.

Zhao, K., Penttinen, P., Guan, T., Xiao, J., Chen, Q., Xu, J., et al. (2011). The diversity and anti-microbial activity of endophytic actinomycetes isolated from medicinal plants in Panxi plateau, China. Curr. Microbiol. 62, 182-190. doi: 10.1007/s00284-010-9685-3

Zhao, K., Ping, W., Li, Q., Hao, S., Zhao, L., Gao, T., et al. (2009). Aspergillus niger var. taxi, a new species variant of taxol-producing fungus isolated from Taxus cuspidata in China. J. Appl. Microbiol. 107, 1202-1207. doi: 10.1111/j.1365-2672.2009.04305.x

Zheng, C.-J., Xu, L.-L., Li, Y.-Y., Han, T., Zhang, Q.-Y., Ming, Q.-L., et al. (2013). Cytotoxic metabolites from the cultures of endophytic fungi from Panax ginseng. Appl. Microbiol. Biotechnol. 97, 7617-7625. doi: 10.1007/s00253-013-5015-6

Zhou, D., Ping, W., Sun, J., Zhou, X., Liu, X., Yang, D., et al. (2001). Isolation of taxol producing fungi. Wei Sheng Wu Xue Za Zhi 21, 18-19, 32.

Zilla, M. K., Qadri, M., Pathania, A. S., Strobel, G. A., Nalli, Y., Kumar, S., et al. (2013). Bioactive metabolites from an endophytic Cryptosporiopsis sp. inhabiting Clidemia hirta. Phytochemistry 95, 291-297. doi: 10.1016/j.phytochem.2013.06.021

Conflict of Interest Statement: The authors declare that the research was conducted in the absence of any commercial or financial relationships that could be construed as a potential conflict of interest.

Copyright @ 2018 Uzma, Mohan, Hashem, Konappa, Rangappa, Kamath, Singh, Mudili, Gupta, Siddaiah, Chowdappa, Alqarawi and Abd_Allah. This is an openaccess article distributed under the terms of the Creative Commons Attribution License (CC BY). The use, distribution or reproduction in other forums is permitted, provided the original author(s) and the copyright owner are credited and that the original publication in this journal is cited, in accordance with accepted academic practice. No use, distribution or reproduction is permitted which does not comply with these terms. 